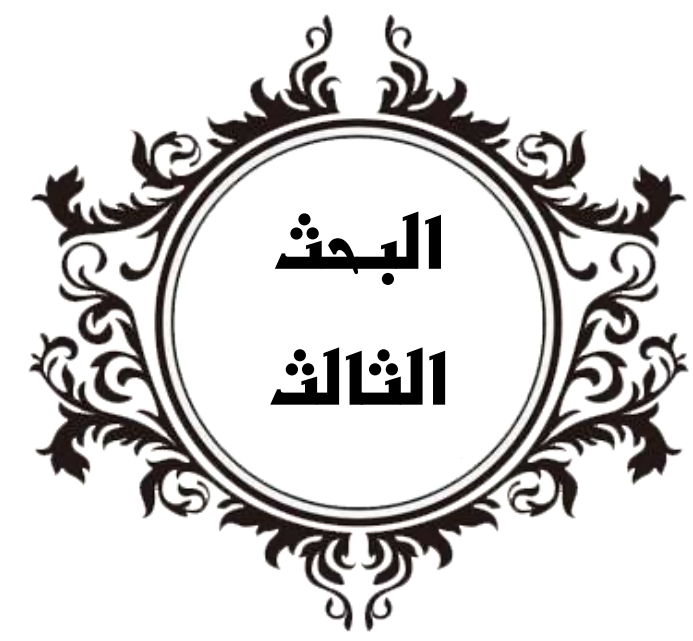

$\square$

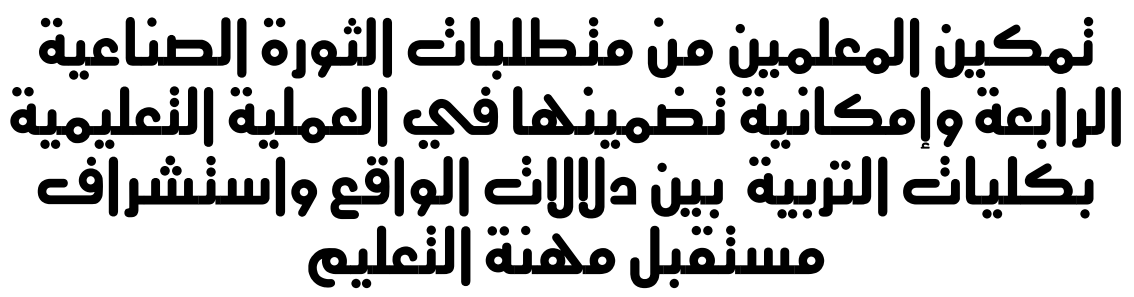

إعــــ|د:

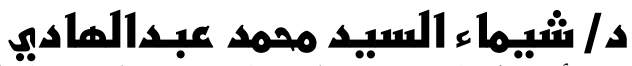
مدرس أصول التربيت كليتت التربيتت جامعت الزّقازيق 



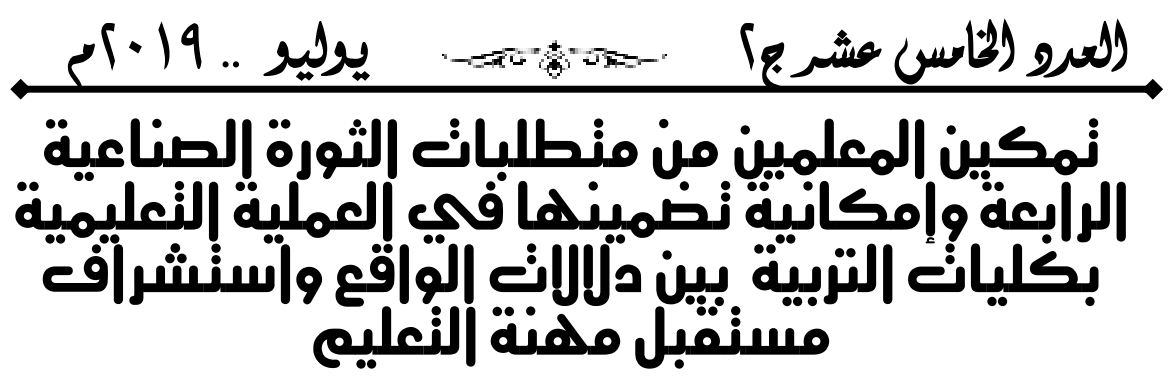

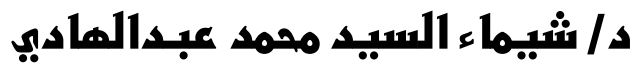 \\ مدرس أصول التربيت كليت التربيتً جامعت الزّبقازيق

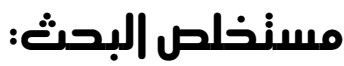

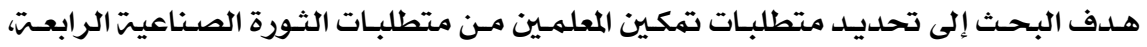

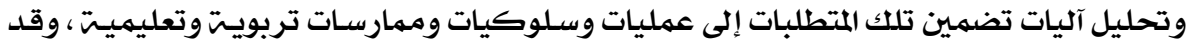

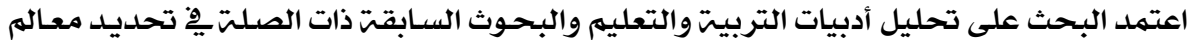

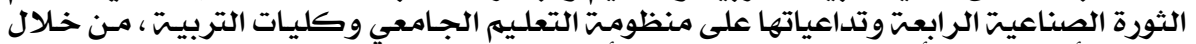

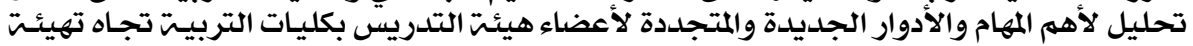

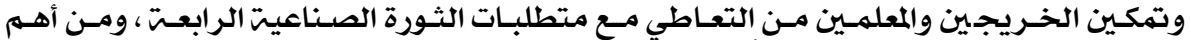

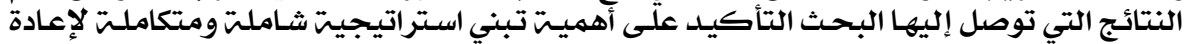

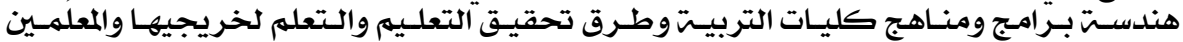

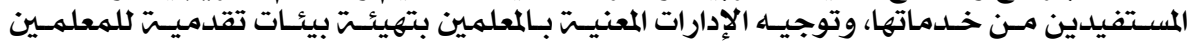

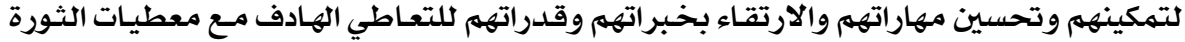

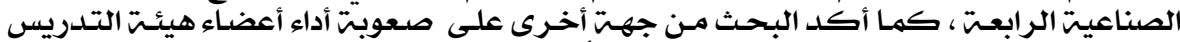

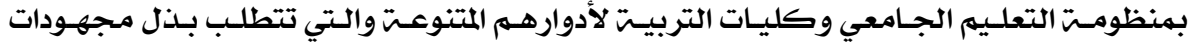

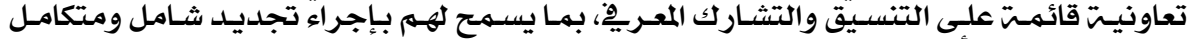

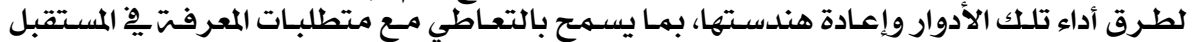

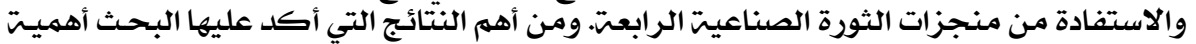

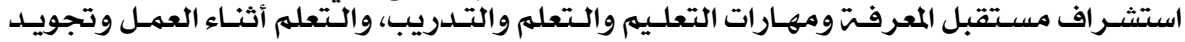

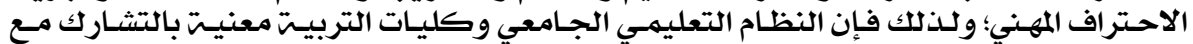

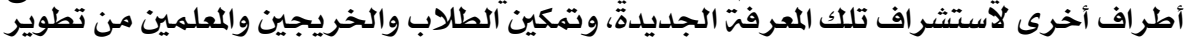

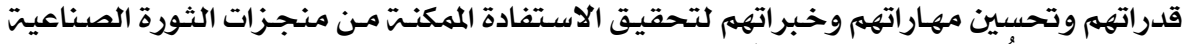

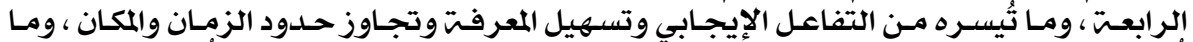

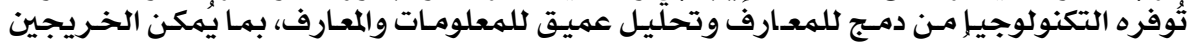

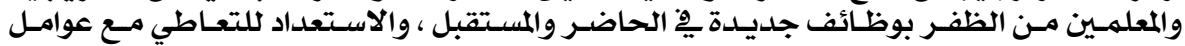

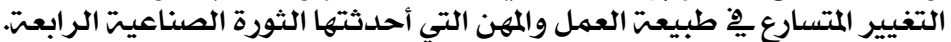

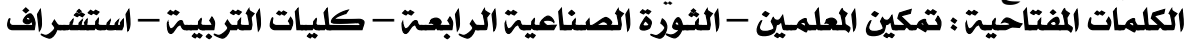
مهنت التعليم ات ماتباحيم.

Empowering Teachers with the Requirements of the Fourth Industrial Revolution and the Possibility of Including it in the Educational Process in the Faculties of Education, among the Indications of Reality and Anticipating the Future of the

\title{
Teaching Profession
}

\section{Dr. Shaimaa El-Sayed Mohamed Abdel-Hadi}

\section{Abstract:}

The aim of the research is to determine the requirements for empowering teachers to meet the requirements of the fourth industrial revolution, and to analyze the mechanisms for including those 


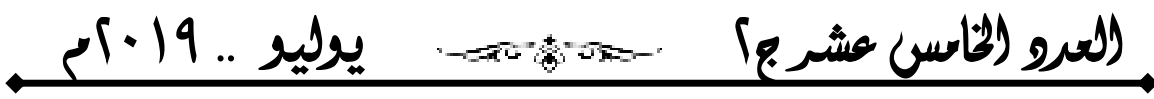

requirements into educational and educational processes, behaviors and practices. The research relied on analyzing the education literature and related previous research in identifying the features of the fourth industrial revolution and its implications for the university education system and colleges of education. Through an analysis of the most important new and renewable tasks and roles of faculty members in the Faculties of Education towards preparing and enabling graduates and teachers to deal with the requirements of the Fourth Industrial Revolution, Among the most important findings of the research is the emphasis on the importance of adopting a comprehensive and integrated strategy to re-engineer the programs and curricula of the Faculties of Education and methods of achieving education and learning for its graduates and teachers who benefit from their services, and directing the departments concerned with teachers to create progressive environments for teachers to enable them, improve their skills, and upgrade their expertise and abilities to deal meaningfully with the data of the Industrial Revolution. Fourth, On the other hand, the research emphasized the difficulty of teaching staff members in the university education system and colleges of education for their various roles, which require cooperative efforts based on coordination and knowledge sharing, in order to allow them to conduct a comprehensive and integrated renewal of the methods of performing those roles and re-engineering them, in order to deal with the requirements of knowledge In the future and benefiting from the achievements of the fourth industrial revolution. Among the most important findings emphasized by the research are the importance of anticipating the future of knowledge, teaching, learning and training skills, learning on the job and improving professionalism. Therefore, the university educational system and colleges of education are concerned with partnering with other parties to explore this new knowledge, and to enable students, graduates and teachers to develop their capabilities and improve their skills and experiences to achieve the possible benefit from the achievements of the Fourth Industrial Revolution. It facilitates positive interaction, facilitates knowledge and transcends the limits of time and space, and the integration of knowledge and deep analysis of information and knowledge provided by technology, enabling graduates and teachers to gain new jobs in the present and the future, and prepare to deal with the factors of rapid change in the nature of work and professions brought about by the Fourth Industrial Revolution.

Keywords: Empowering Teachers - Fourth Industrial Revolution- Faculties of Education= Anticipating the Future of the Teaching Profession
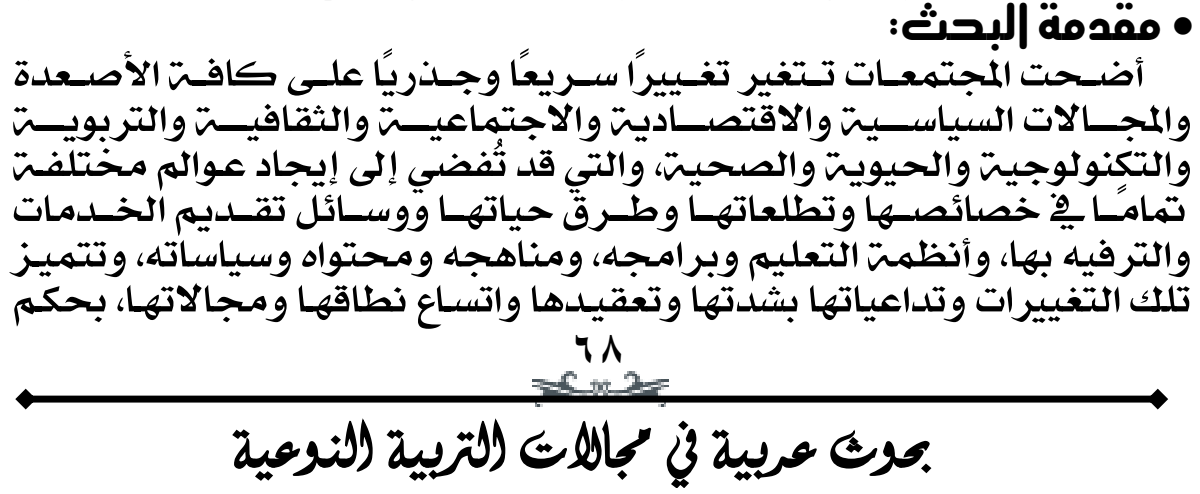


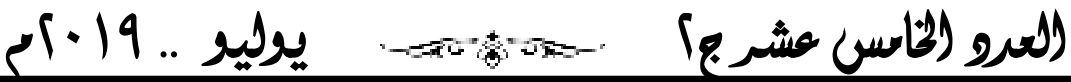

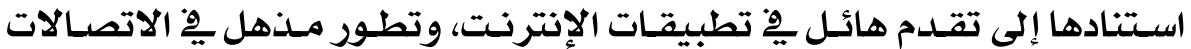

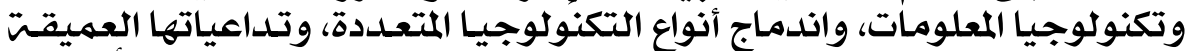

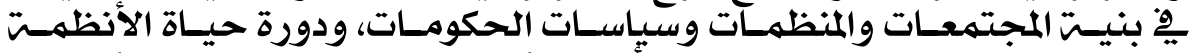

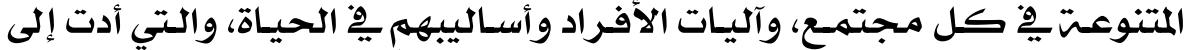

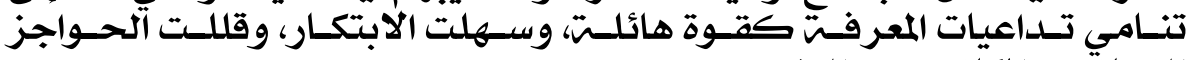

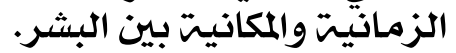

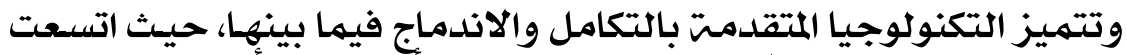

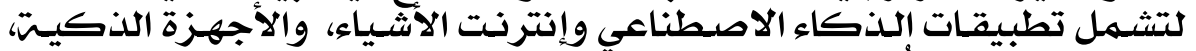

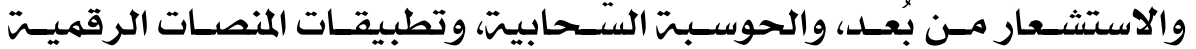

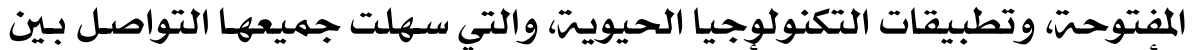

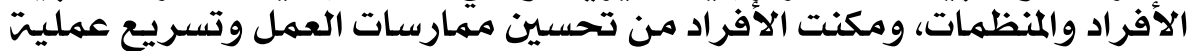

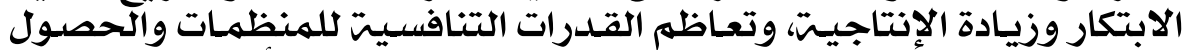

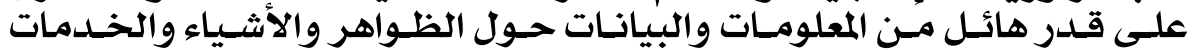

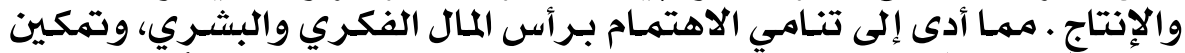

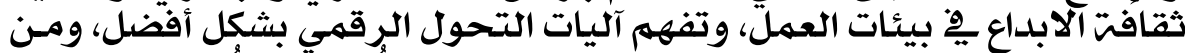

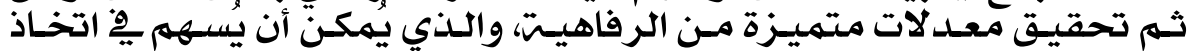

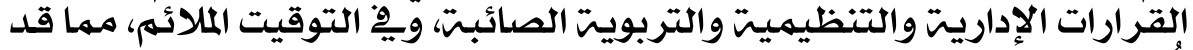

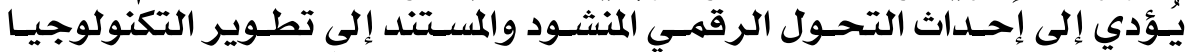

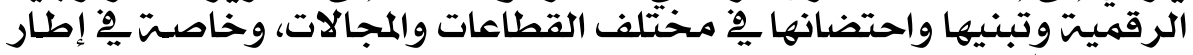

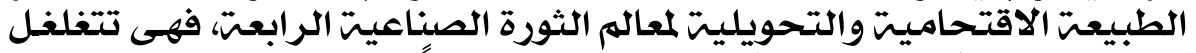

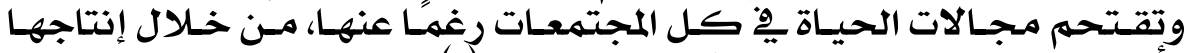

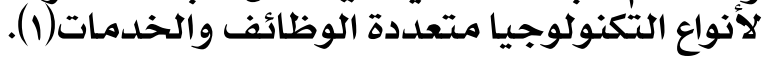

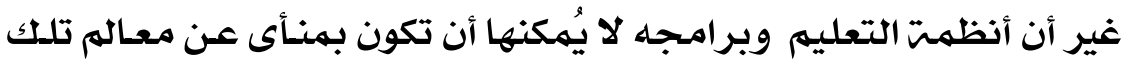

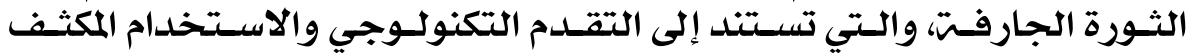

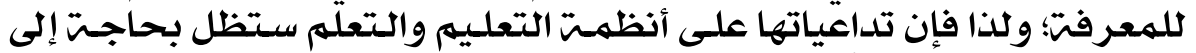

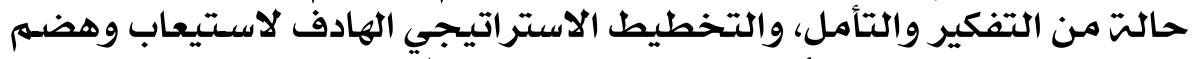

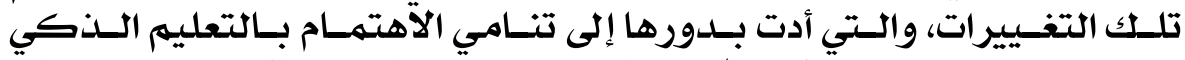

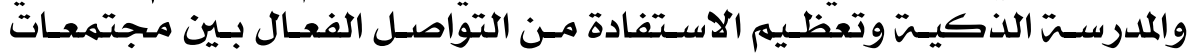

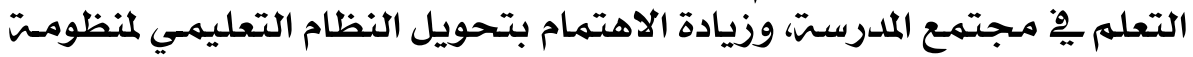

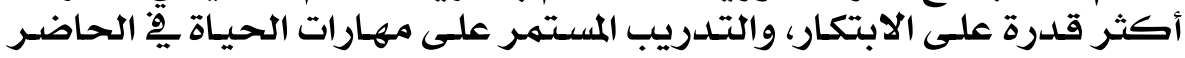

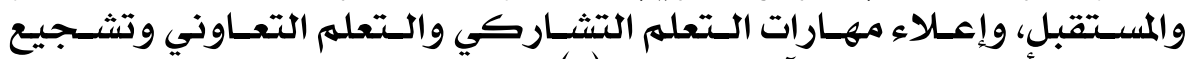

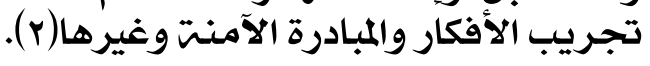

1- Verina, N.; Titko, J. (2019). Digital Transformation: Conceptual Framework. International Scientific Conference. Contemporary Issues in Businessm Management and Economics Engineering. 9-10 May, Vilnius, Lithuania. Vilnius Gediminas Technical University, 720.

2-Aida Aryani Shahroom, Norhayati Hussin(2018. September). Industerial Revolution 4.0 and Education. International Journal of Academic Research in Business and Social Sciences,8(9), Human Resource Management Academic Research Society, 316.. 


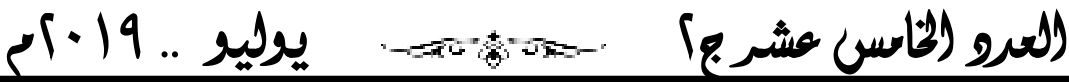

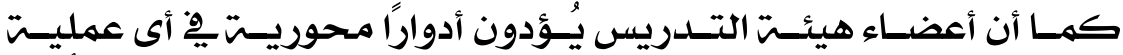

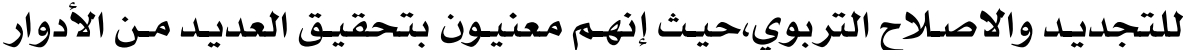

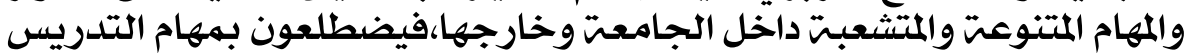

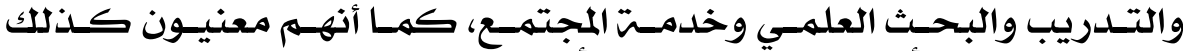

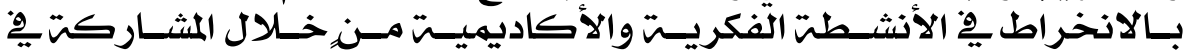

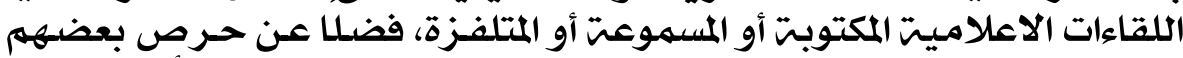

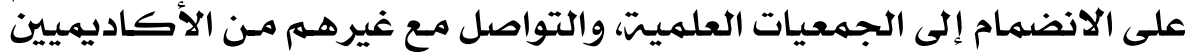

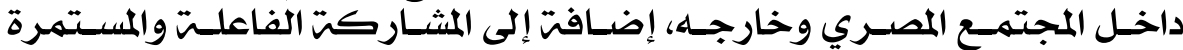

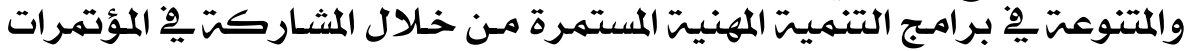

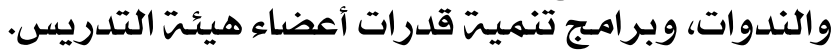

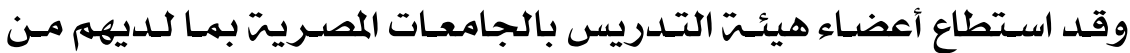

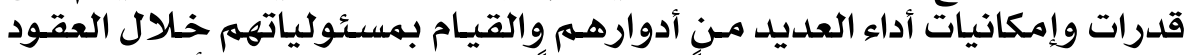

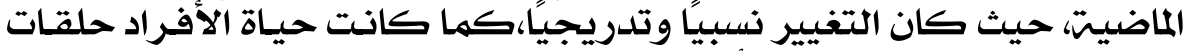

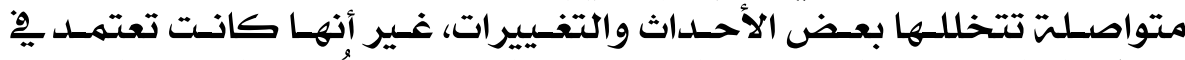

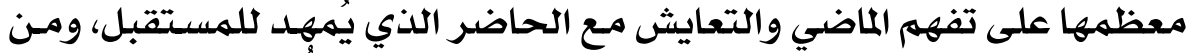

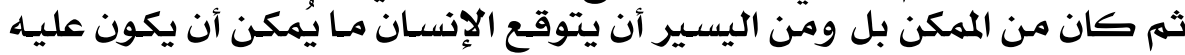

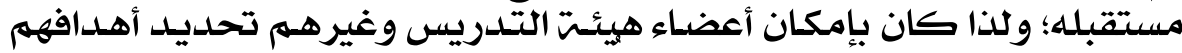

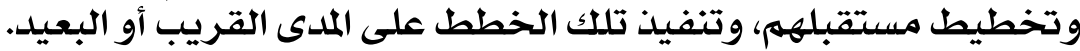

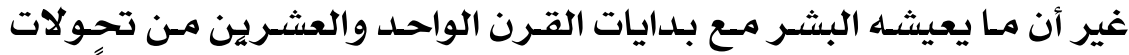

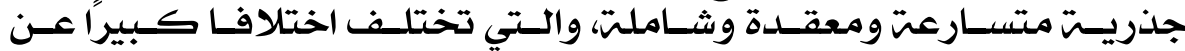

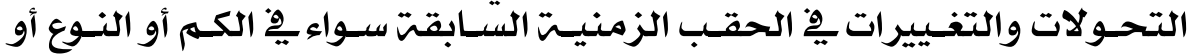

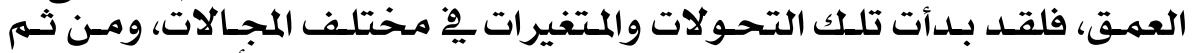

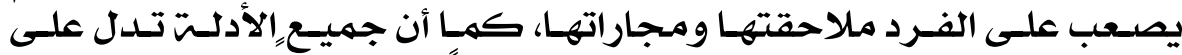

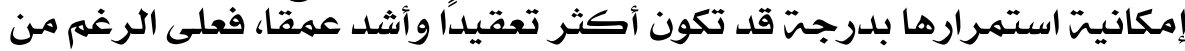

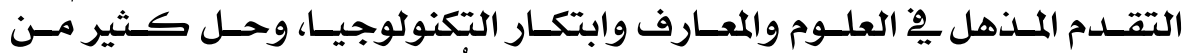

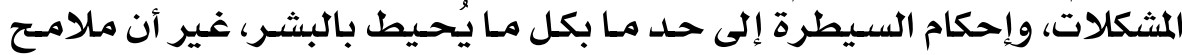

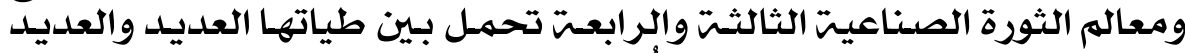

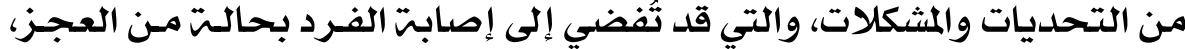

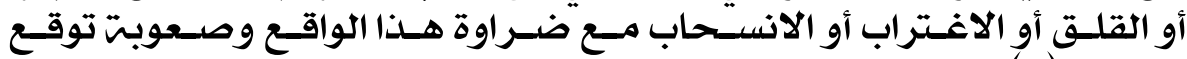

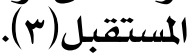

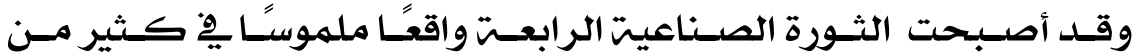

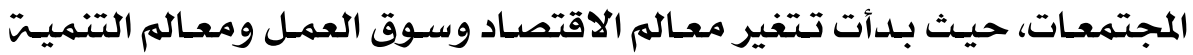

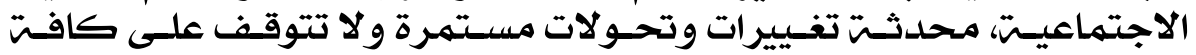

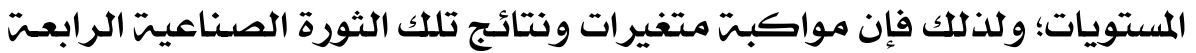

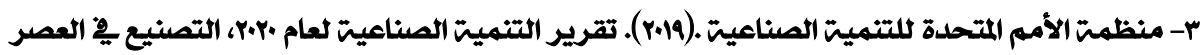
V.

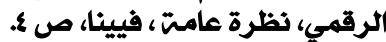




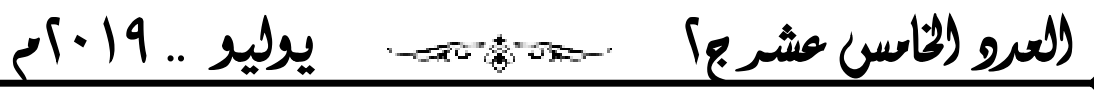

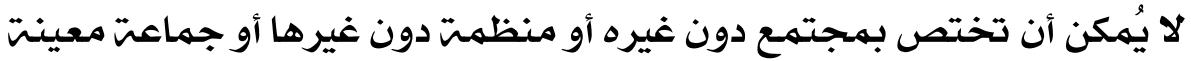

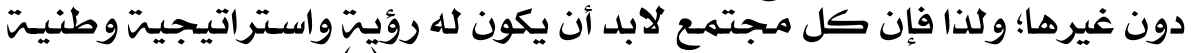

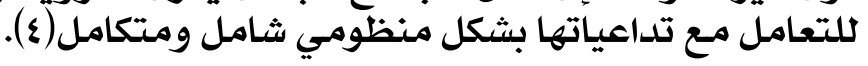

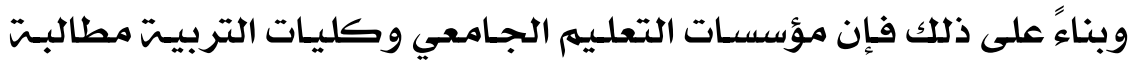

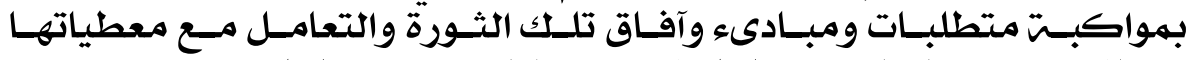

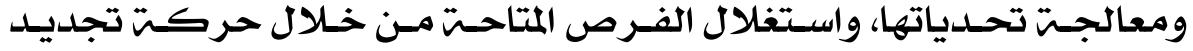

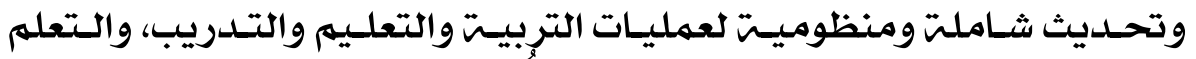

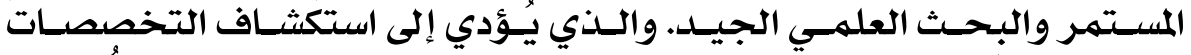

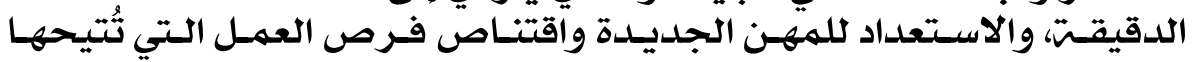

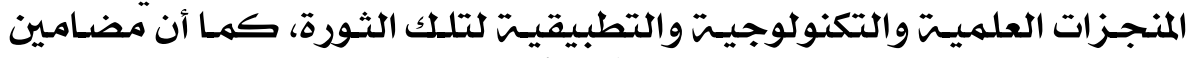

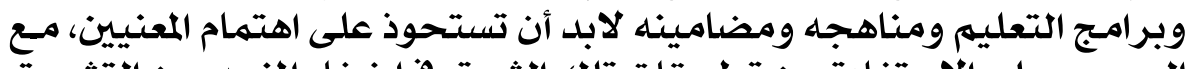

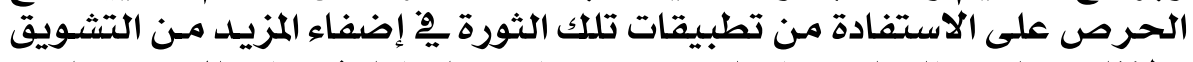

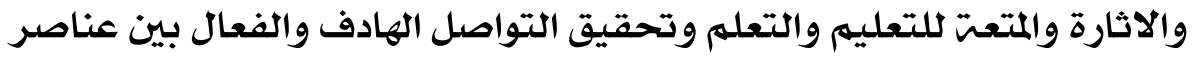

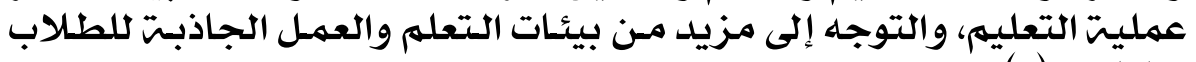

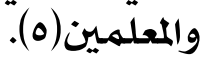

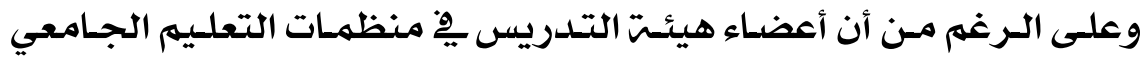

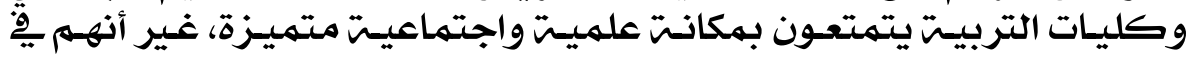

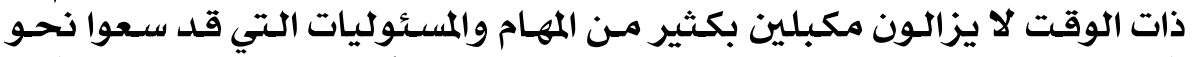

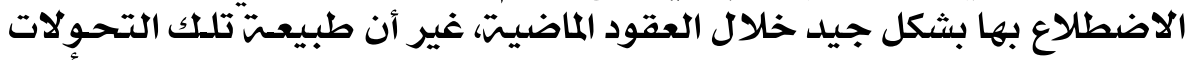

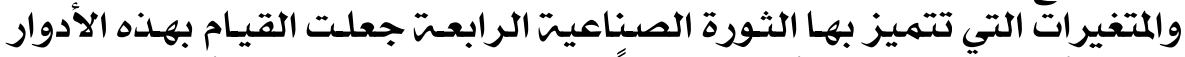

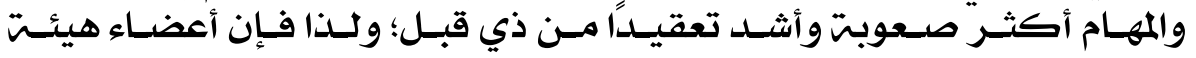

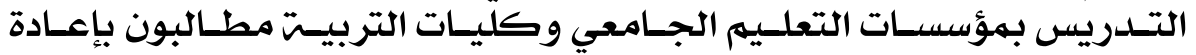

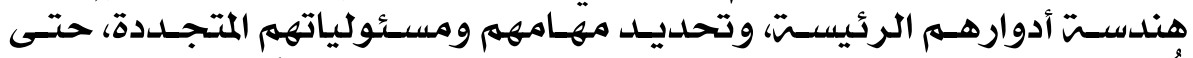

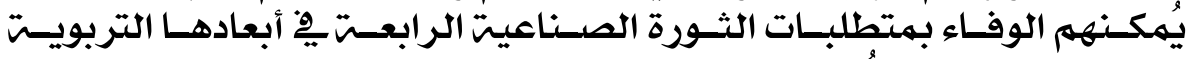

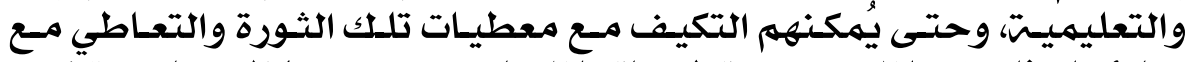

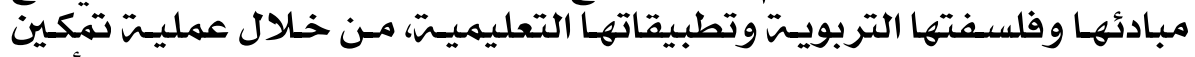

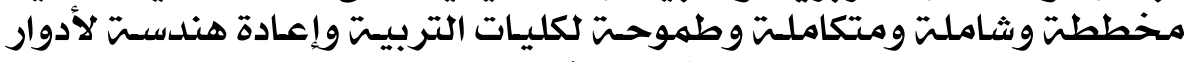

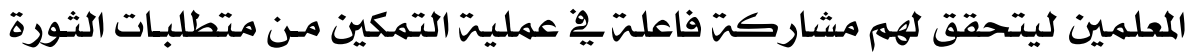

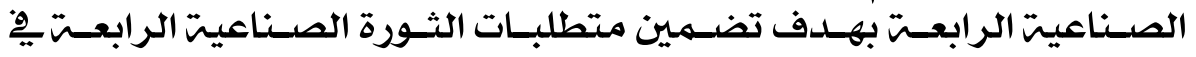

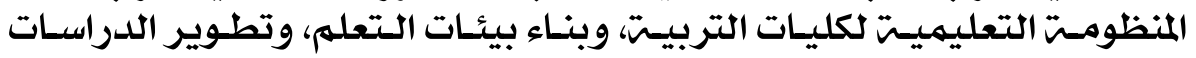

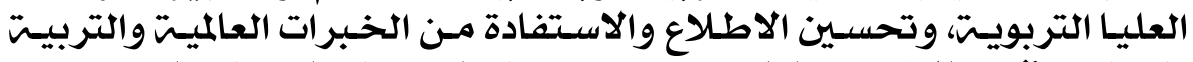

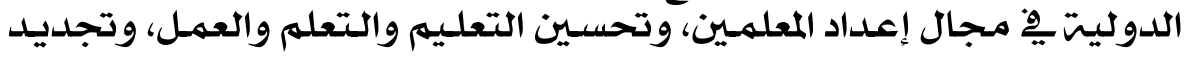

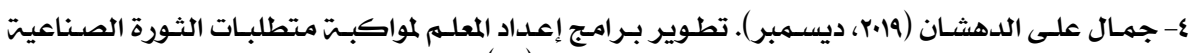

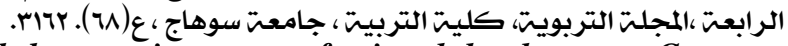
5- Machis, D. (2019). Mind the gaps in your professional development, Contract Management ، 59، p.87 Retrieved from https://search. proquest.com/docview/ 2173405575? accountid $=63189$ 


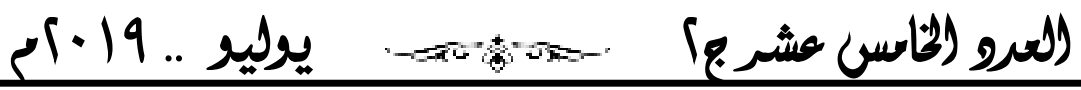

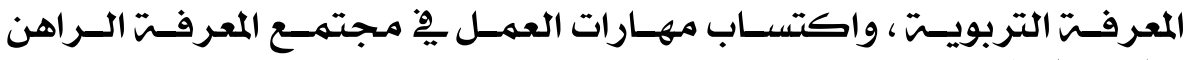
والمستقبل القريب.

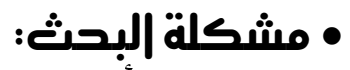

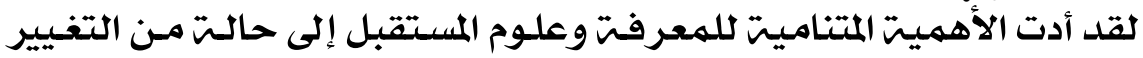

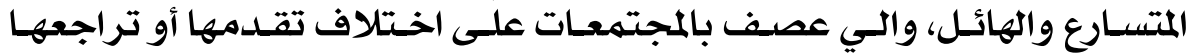

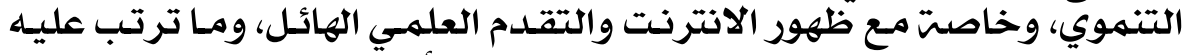

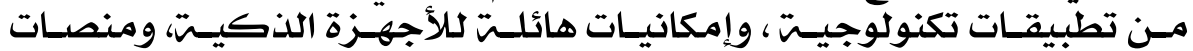

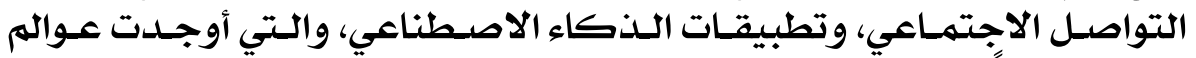

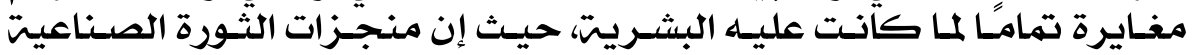

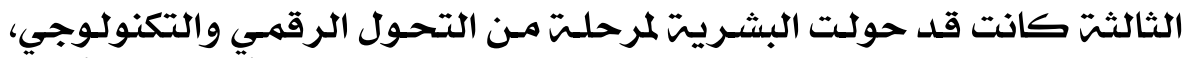

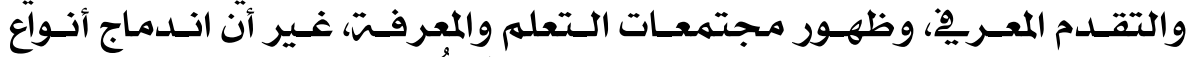

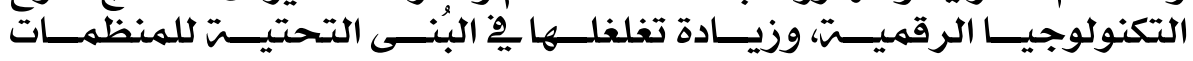

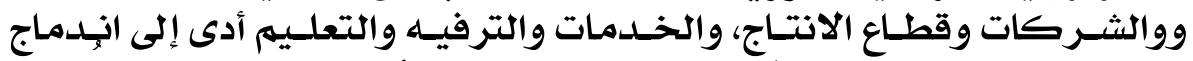

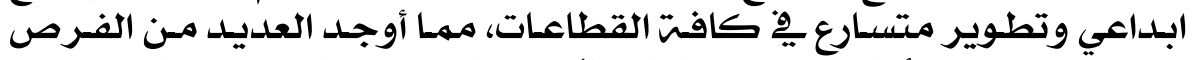

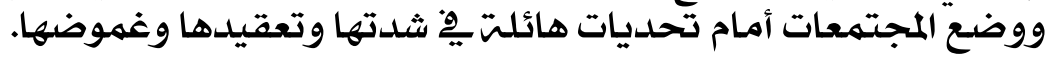

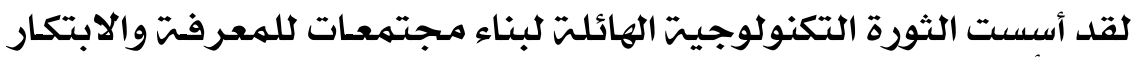

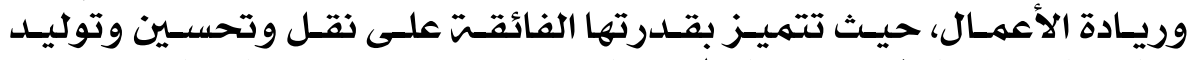

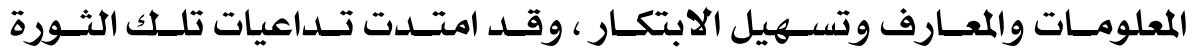

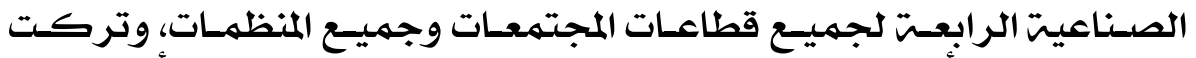

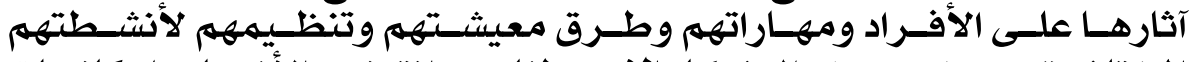

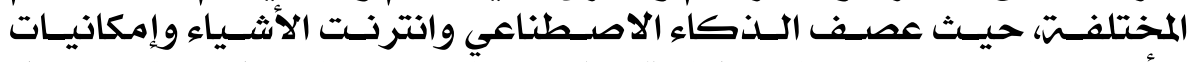

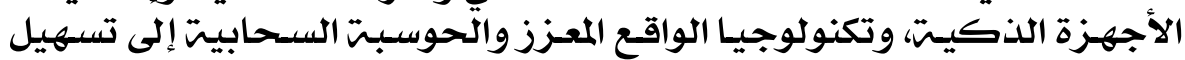

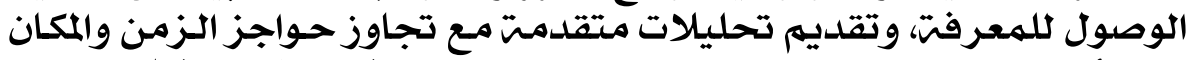

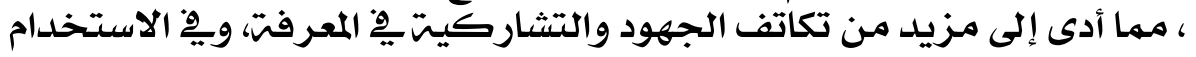

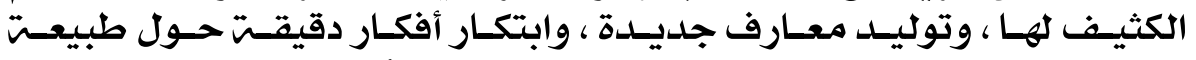

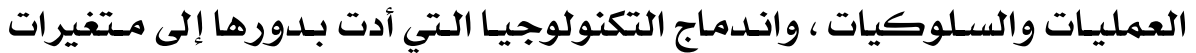

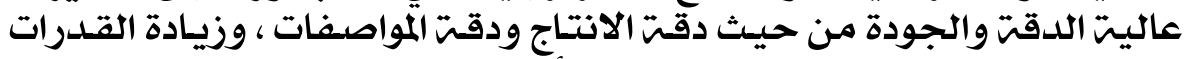

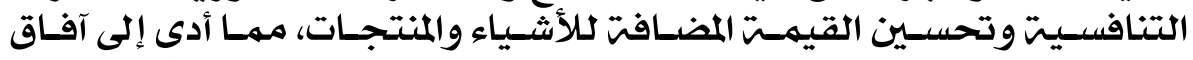

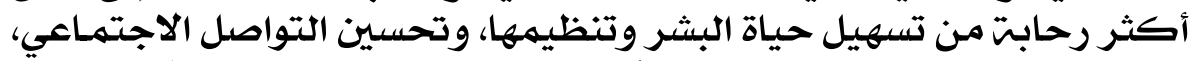

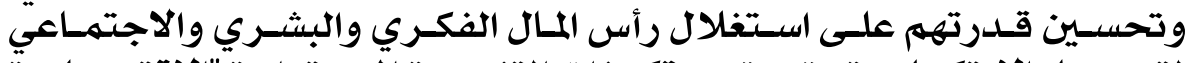

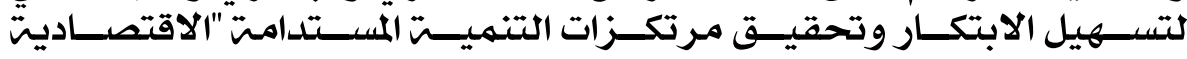

$$
\text { والاجتماعيت والبيئيت". }
$$

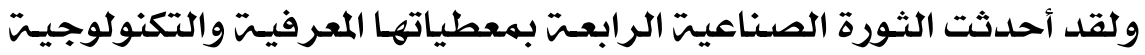

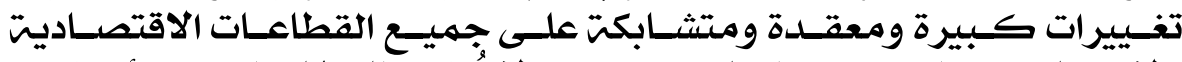

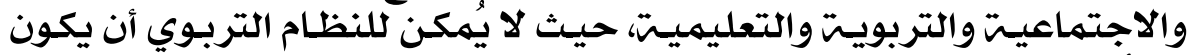

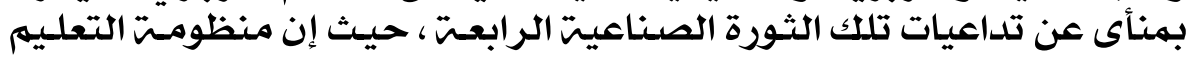




\section{(العرو (لإمسر) عشرج) .}

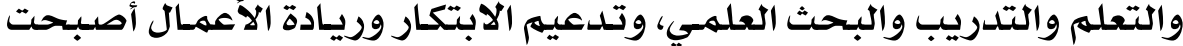

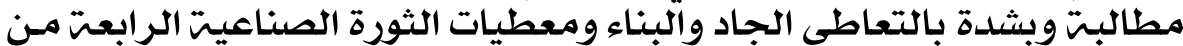

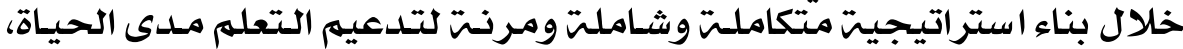

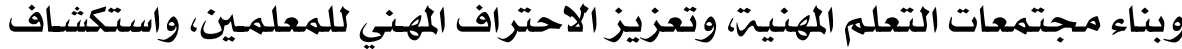

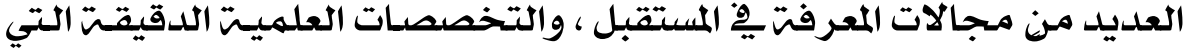

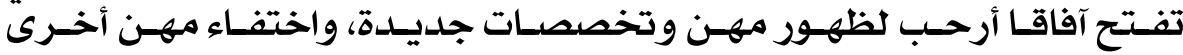

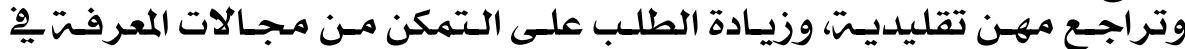

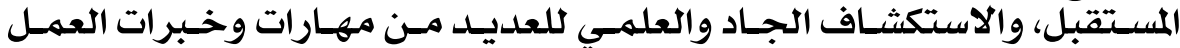

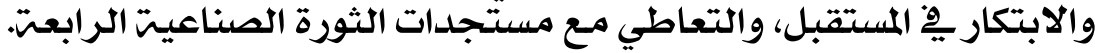

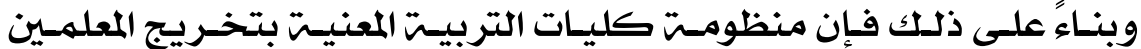

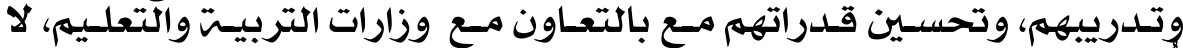

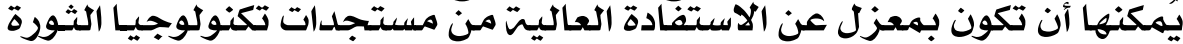

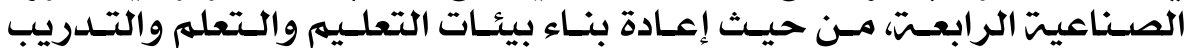

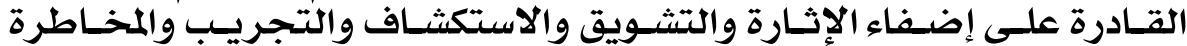

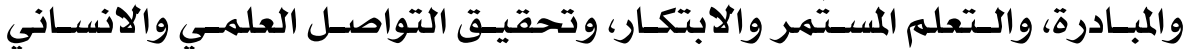

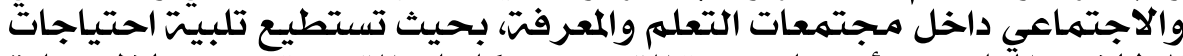

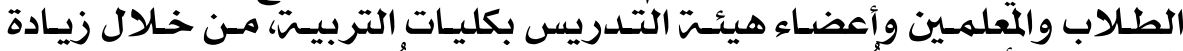

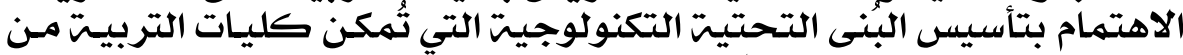

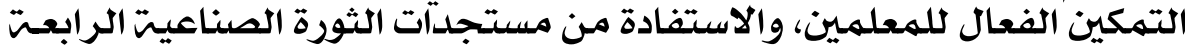

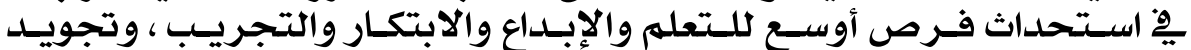

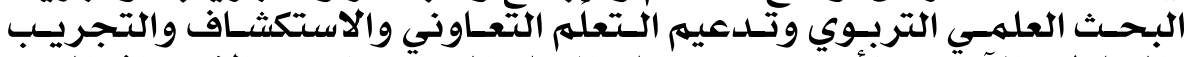

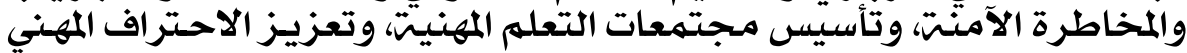

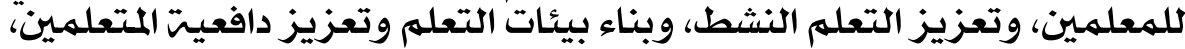

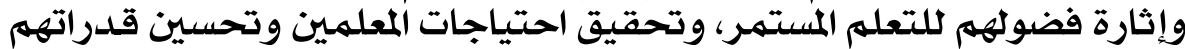

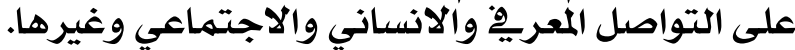

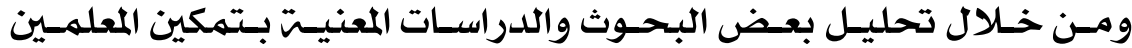

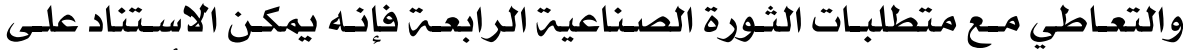

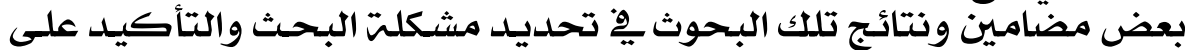

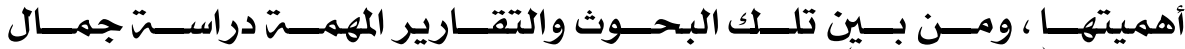

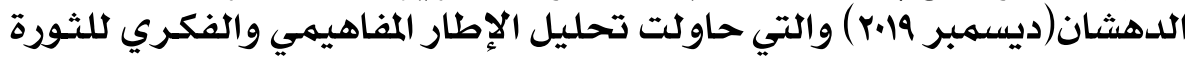

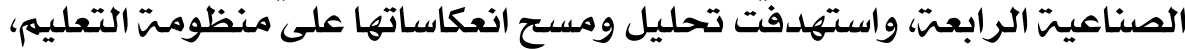

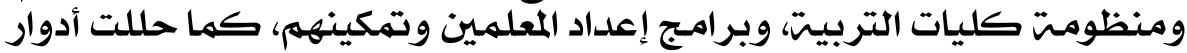

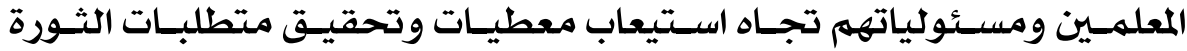

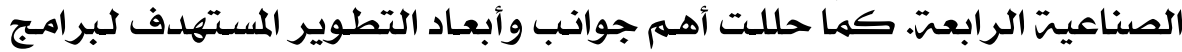

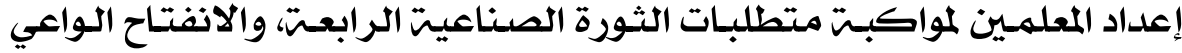

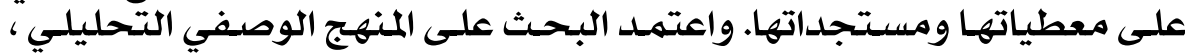

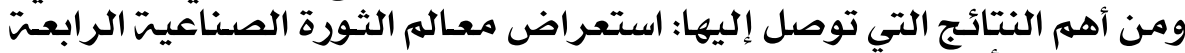

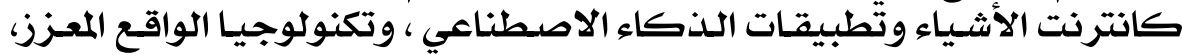




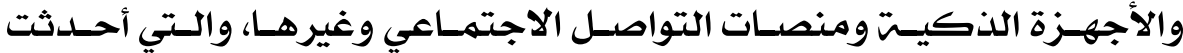

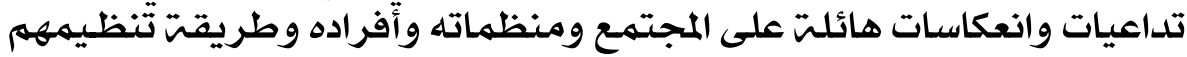

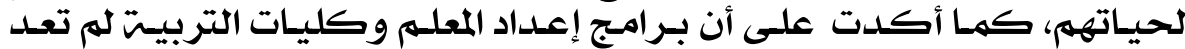

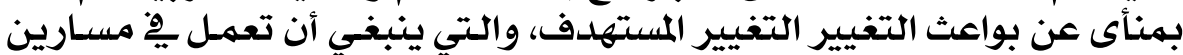

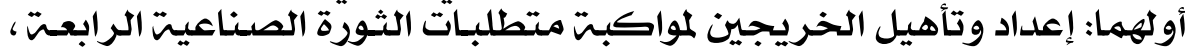

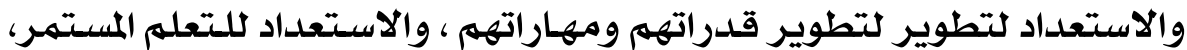

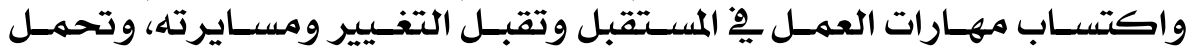

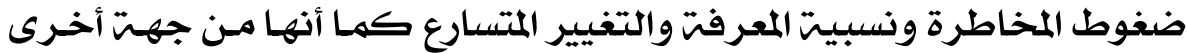

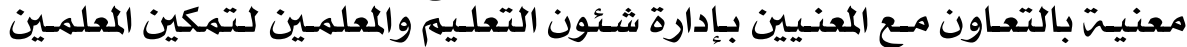

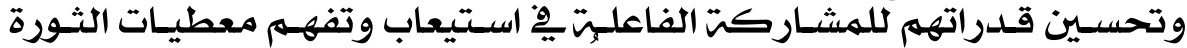

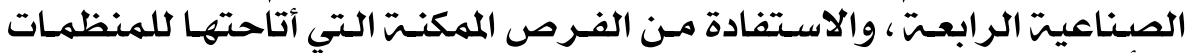

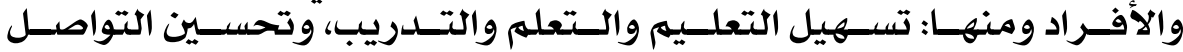

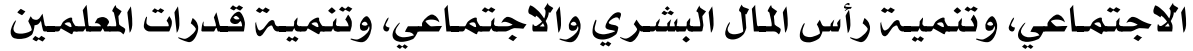

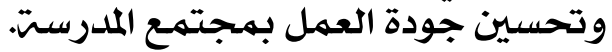

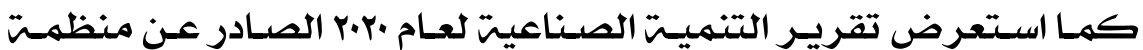

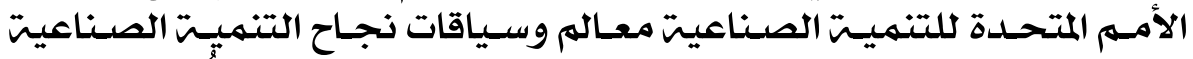

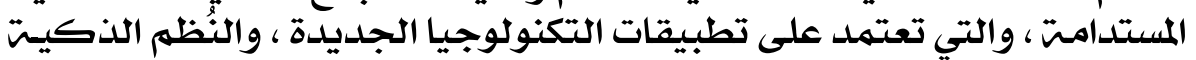

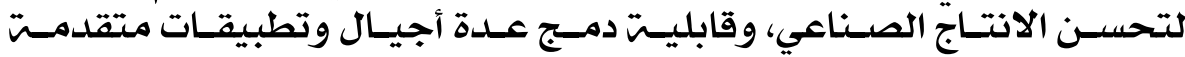

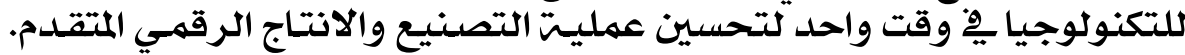

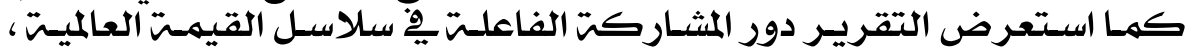

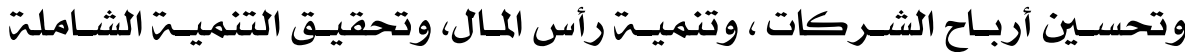

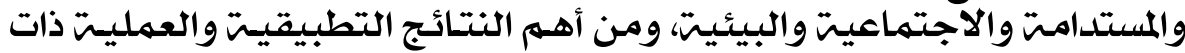

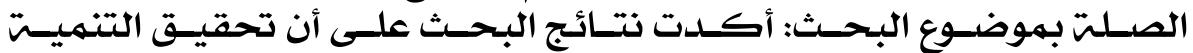

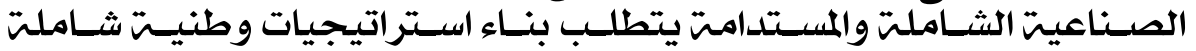

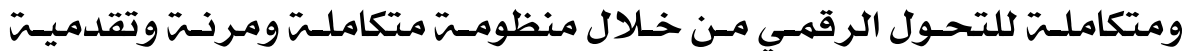

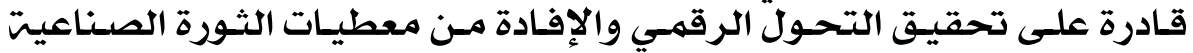

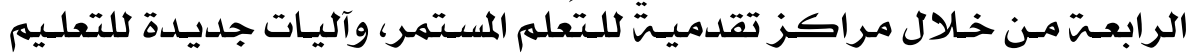

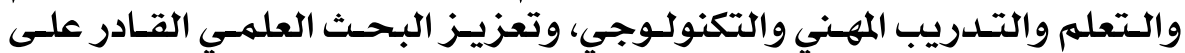

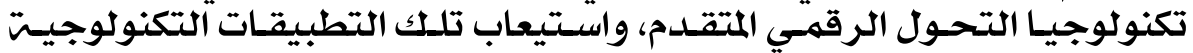

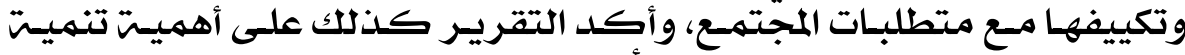

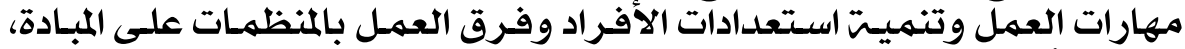

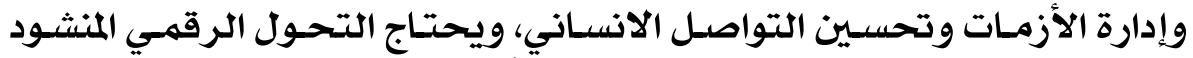

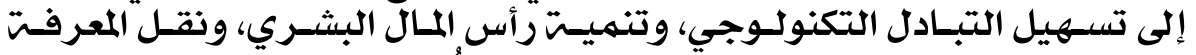

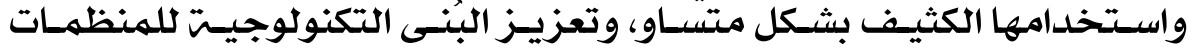

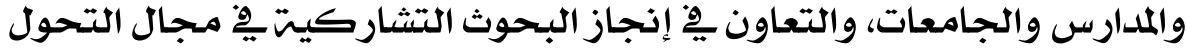

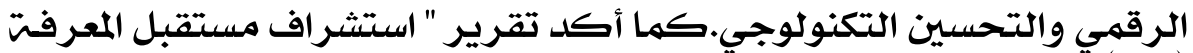

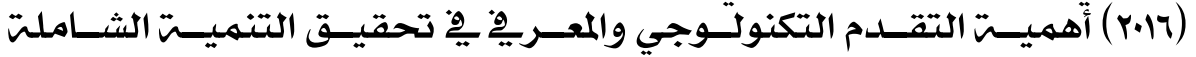




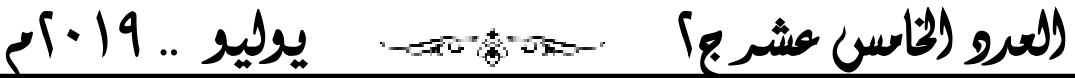

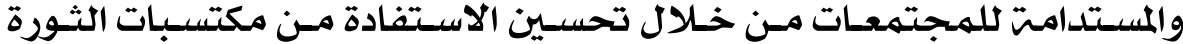

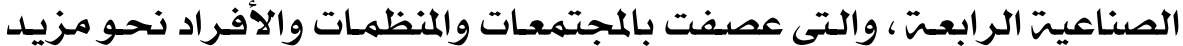

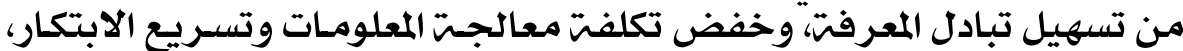

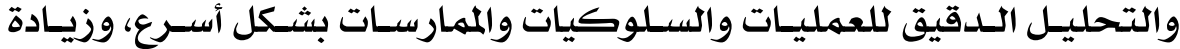

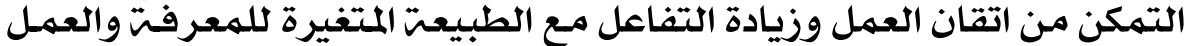
واستيعاب المهارات الجديلدة التي يتطلبهان التهل التوق التهاع العهل.

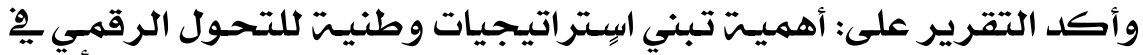

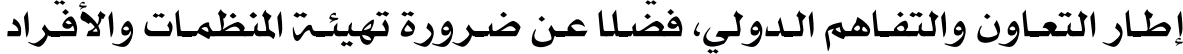

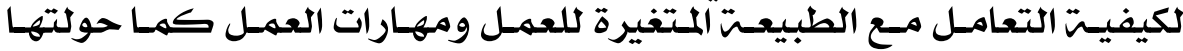

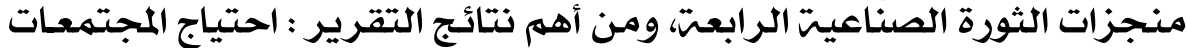

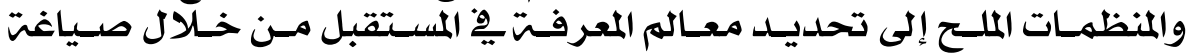

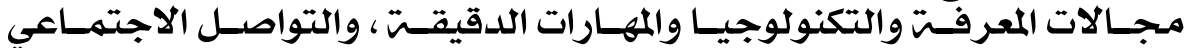

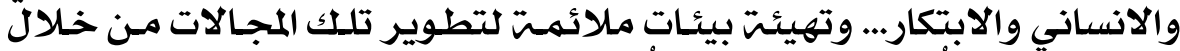

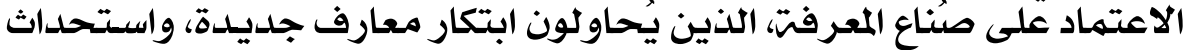

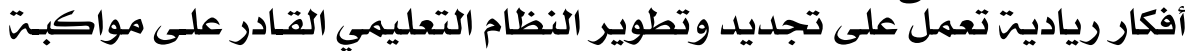

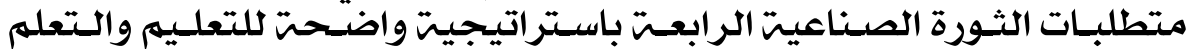

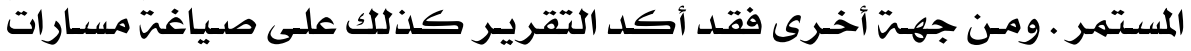

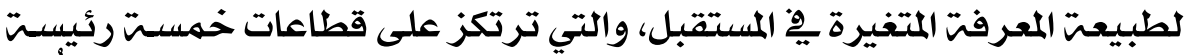

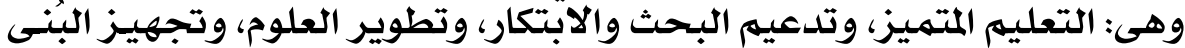

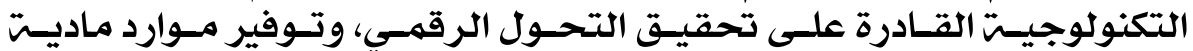

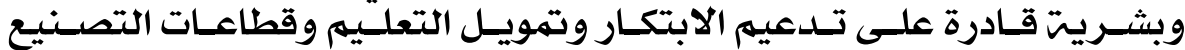

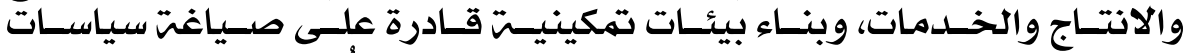

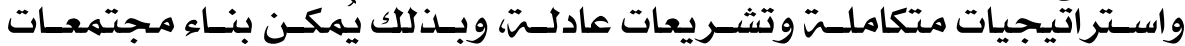

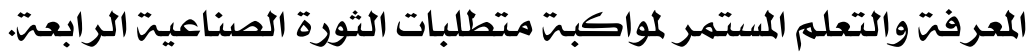

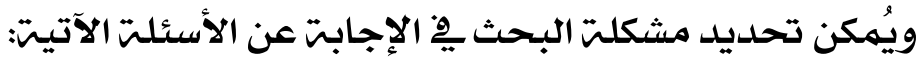

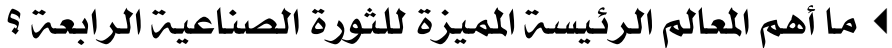

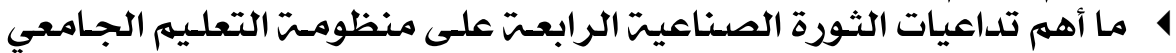

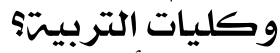

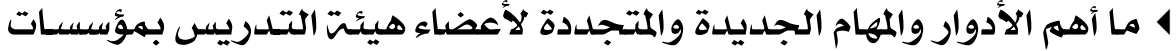

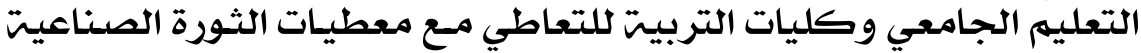

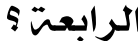

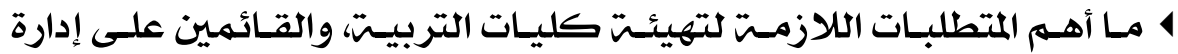

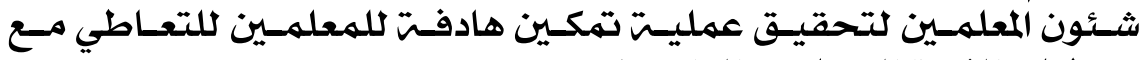

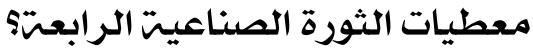

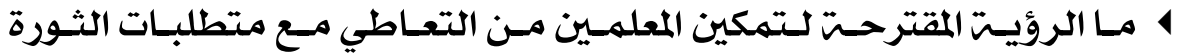

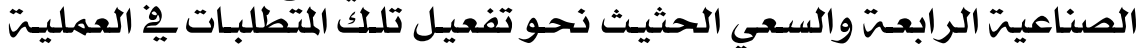

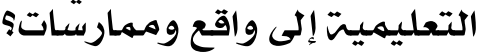




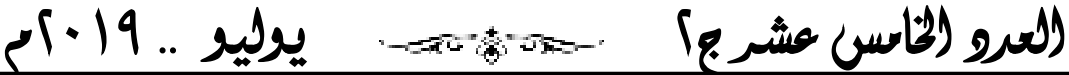

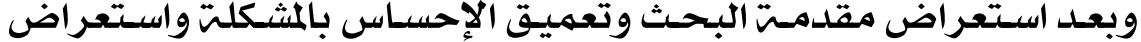

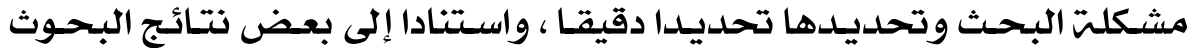

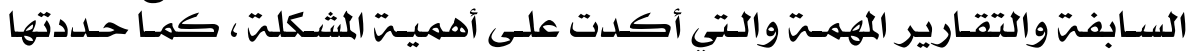

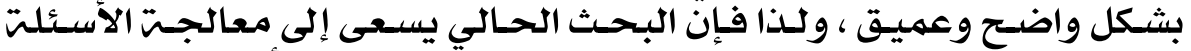

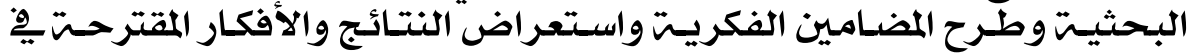

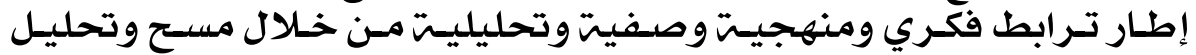

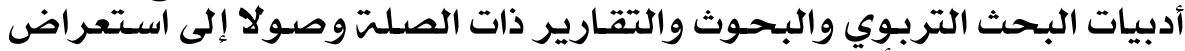

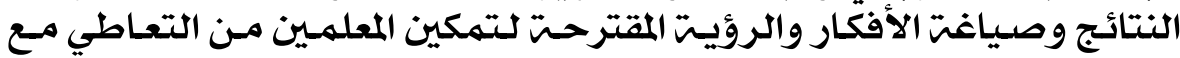

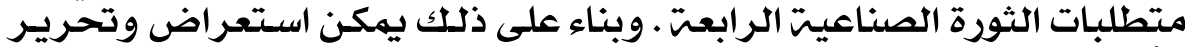

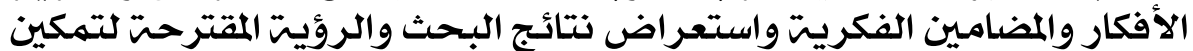

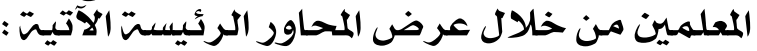

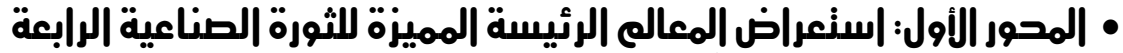

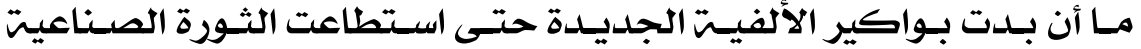

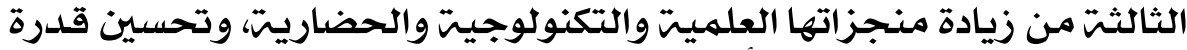

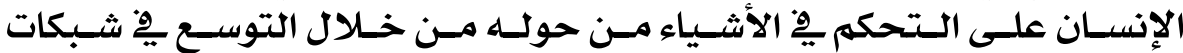

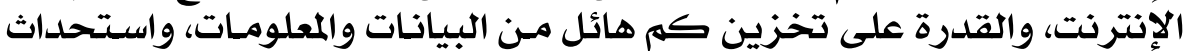

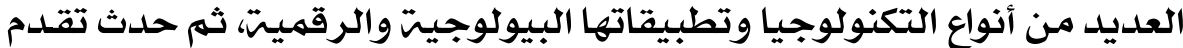

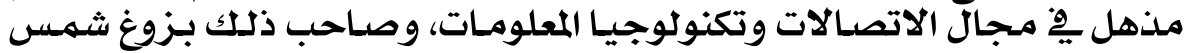

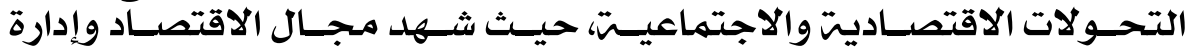

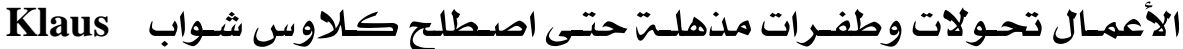
والتي Shwap

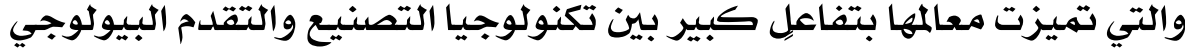

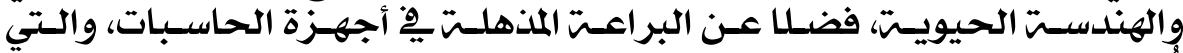

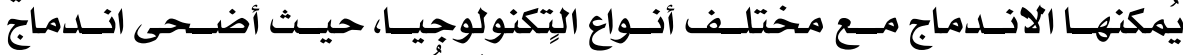

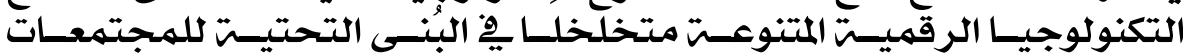

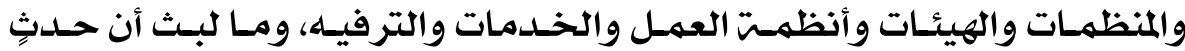

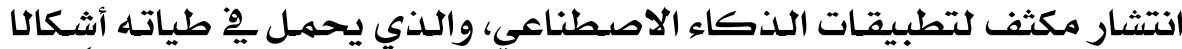

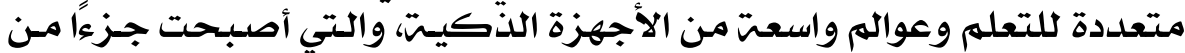

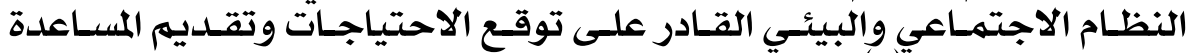
للفرد عند الحاجت" (7).

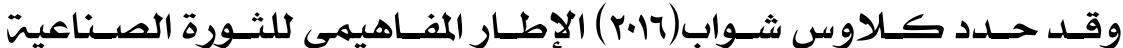

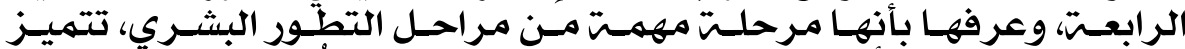

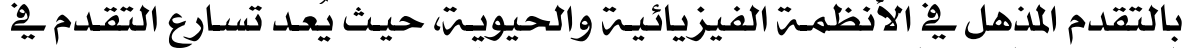

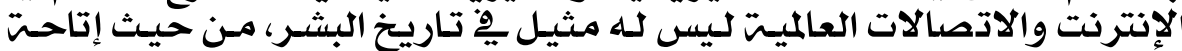

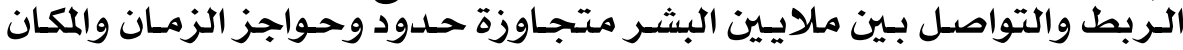

6- Klaus Schwab.(2016). The Fourth Industerial Revolution . Switzerland: World Economic Forum, p15. 


\section{(العرو (لأاسم) عشرج) .}

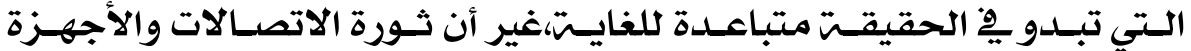

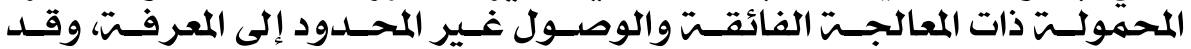

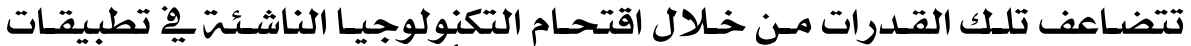

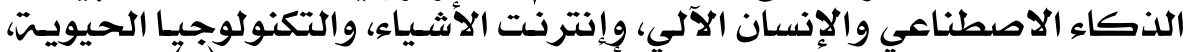

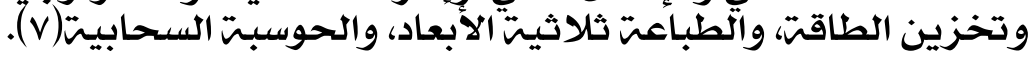

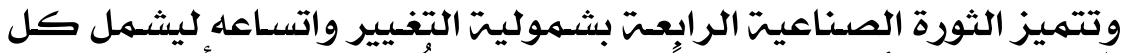

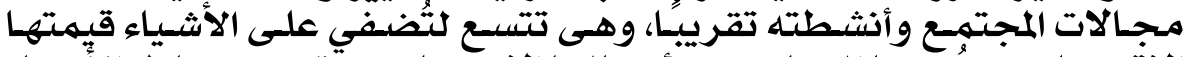

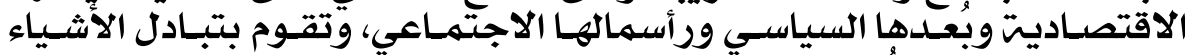

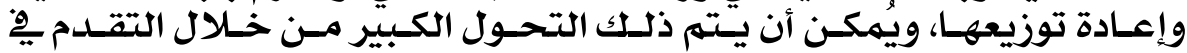

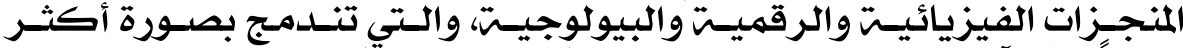

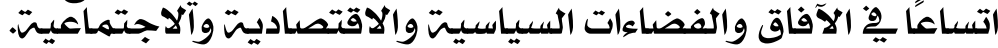

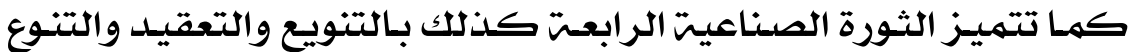

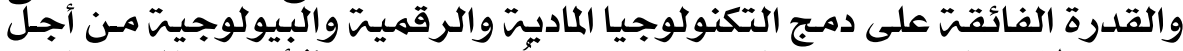

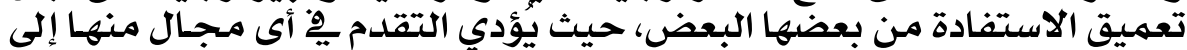

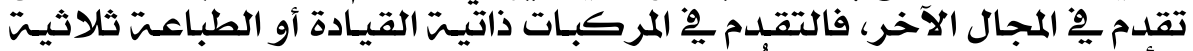

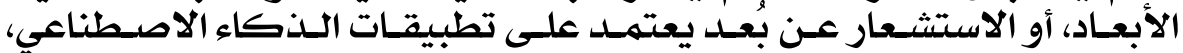

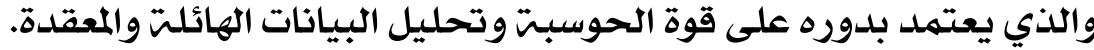

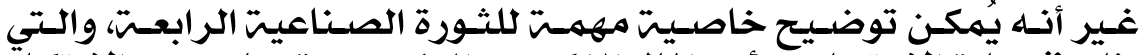

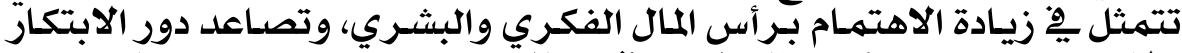

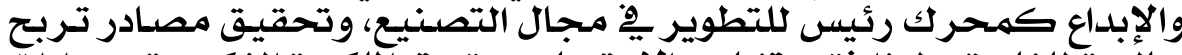

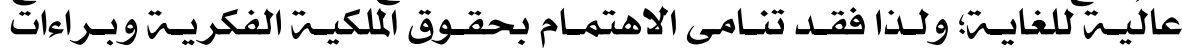

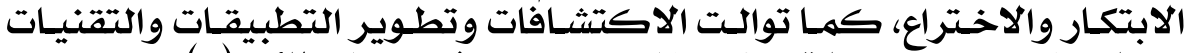

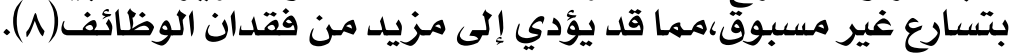

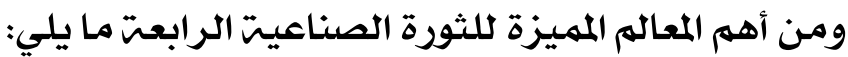

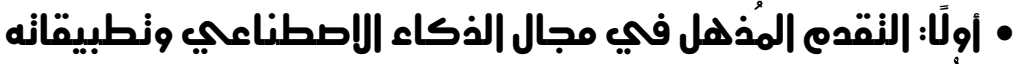

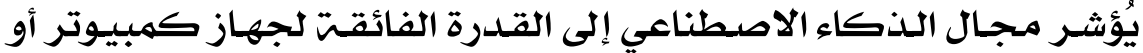

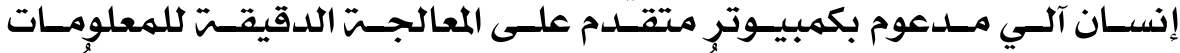

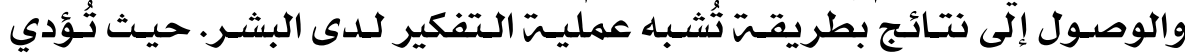

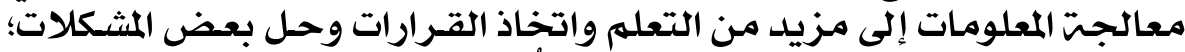

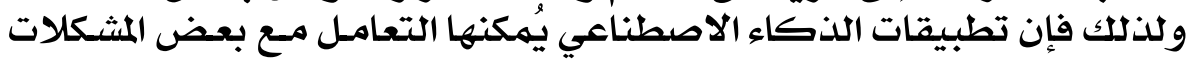

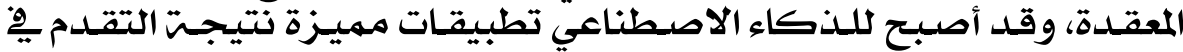

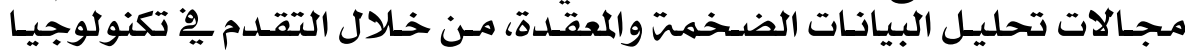

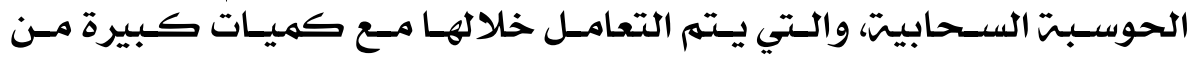

\section{7- Ibid,p.16.}

8- Brown-martin,g(2017). Education and the fourth industrial Revolution. UK;Groube Media tfo, P.11. 


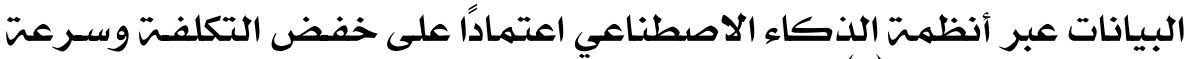

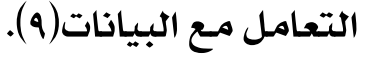

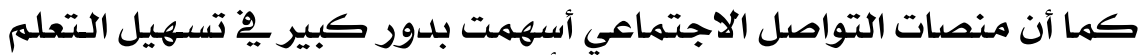

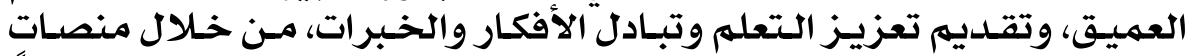

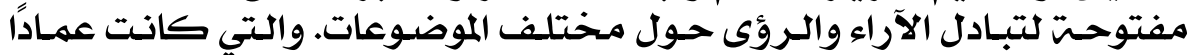

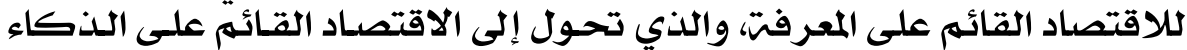

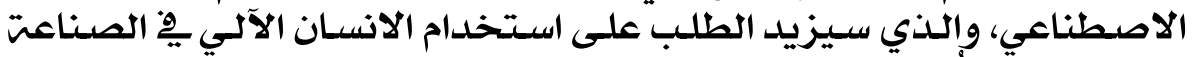

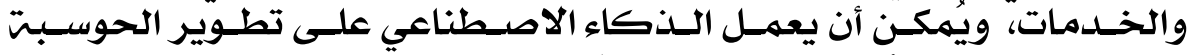

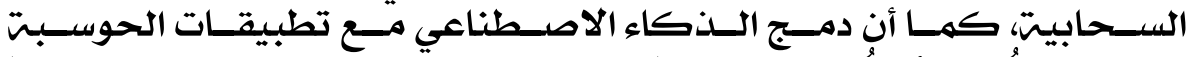

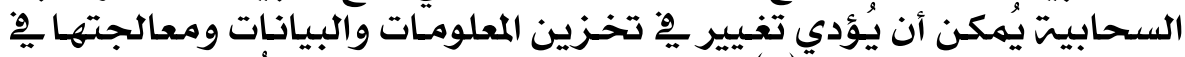

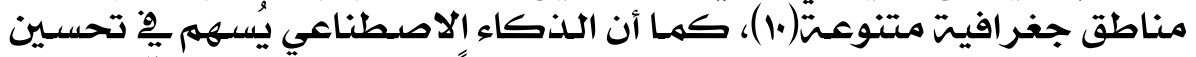

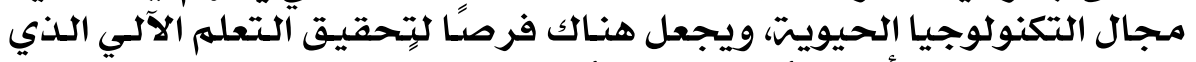

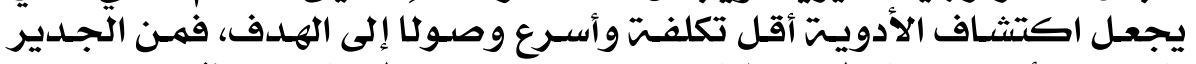

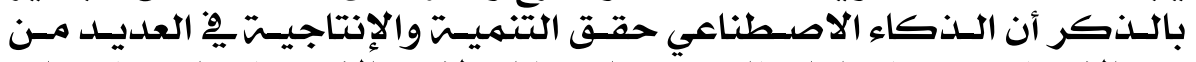

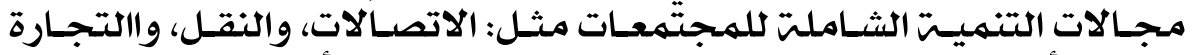

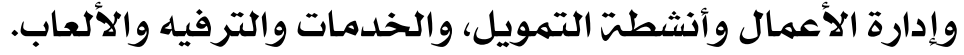

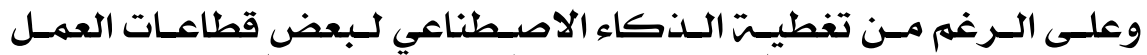

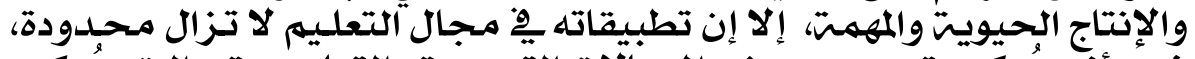

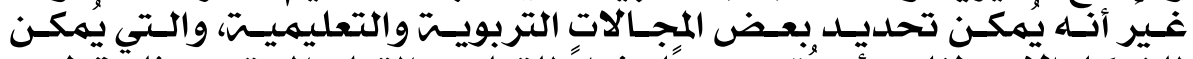

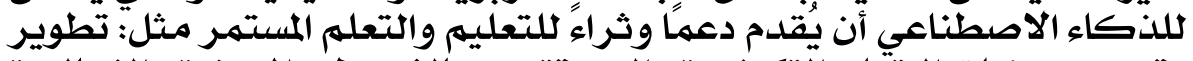

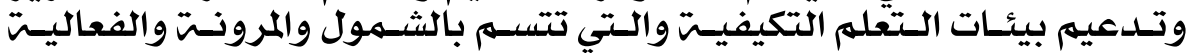

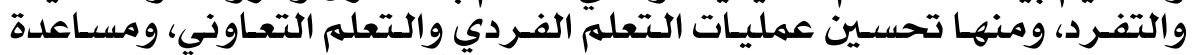

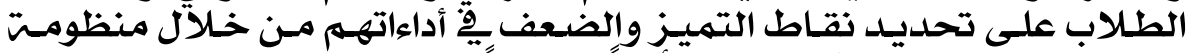

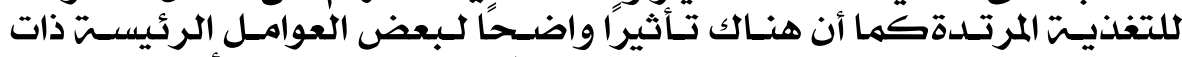

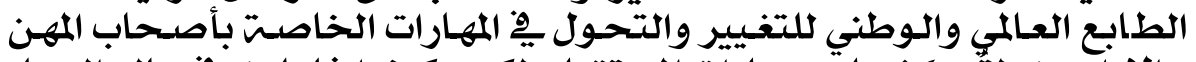

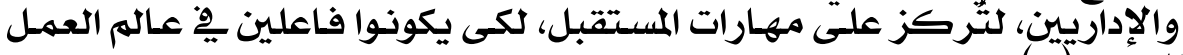
الجديد(11). (11).

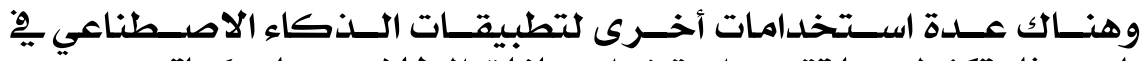

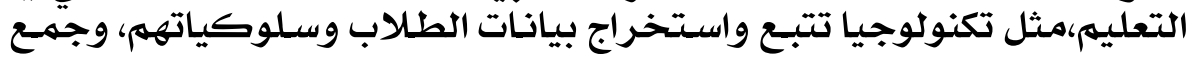

9. Pombo, C. ; Gupta, R. ; Stankovic, M.(2018). Social Services for digital Citizens Opportunities for Latin America\& the Caribbean. Inter- American Development Bank Felipe Herrera Liberary, P.7.

10- Zouein, P (2019). Higher education on 4.0 Drivers and Framework, Expert Group Meeting on " Artificial Intelligence and Local Industrial Development, Lebanese American University, UN-House, Beirut, P.8.

11-Venter, A. A. J., Herbst, T. H. H., \& Iwu, C. G. (2019). What will it take to make a successful administrative professional in the fourth industrial revolution? SA Journal of Human Resource Management 17, doi:http://dx.doi.org /10.4102/ sajhrm.v17i0.1224. 


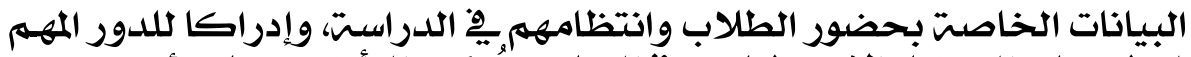

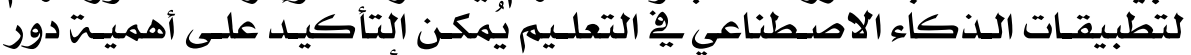

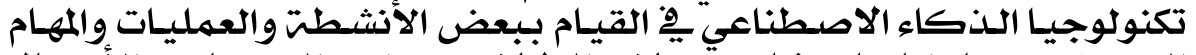

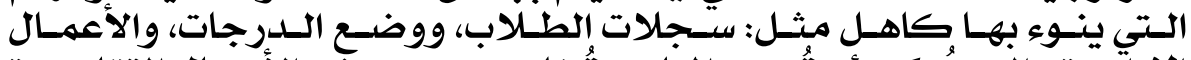

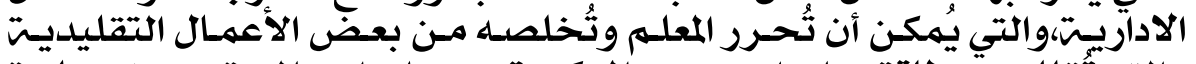

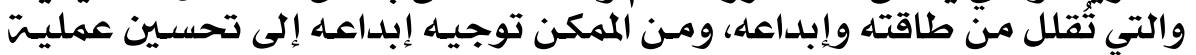

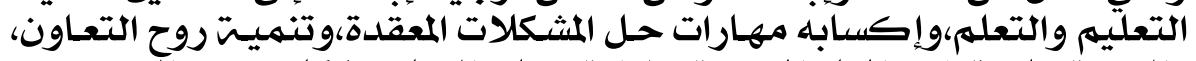

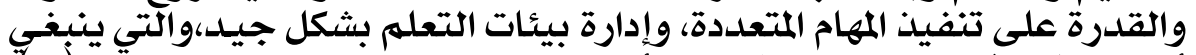

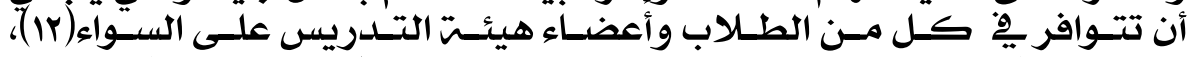

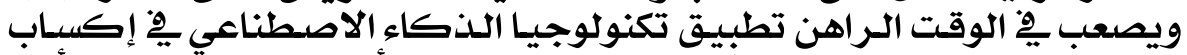

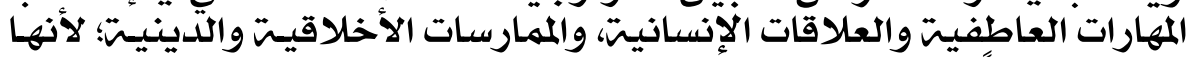

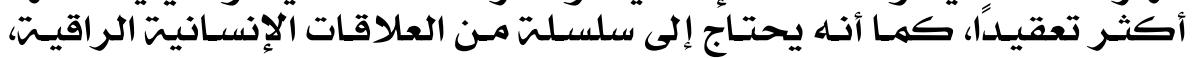

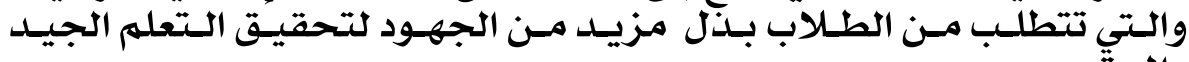
والمستثمر. تَتهر.

\section{• ثانيًا: نوظيفه إنتزننه الأشياء في عملينه النمليه والنمله}

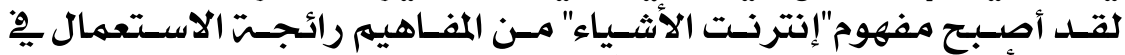

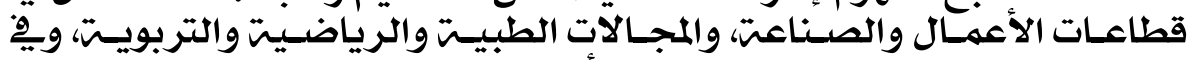

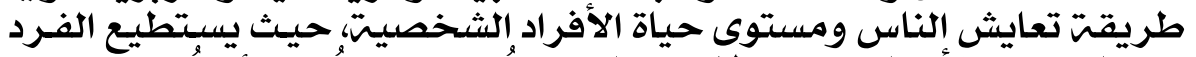

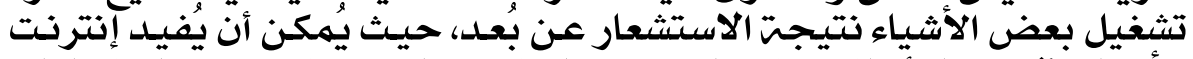

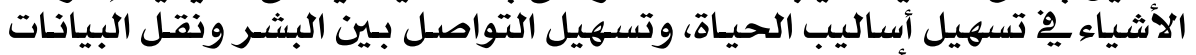

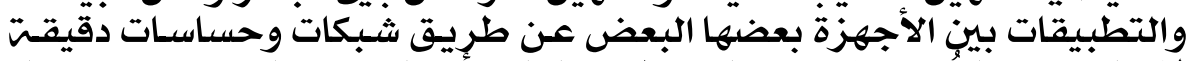

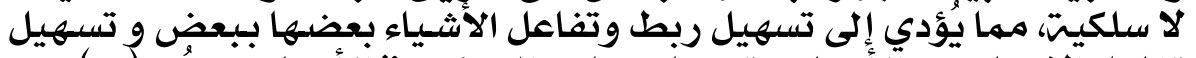

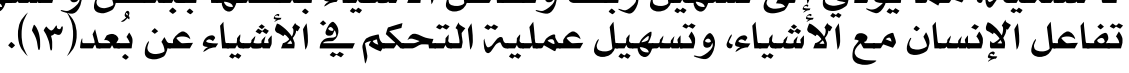

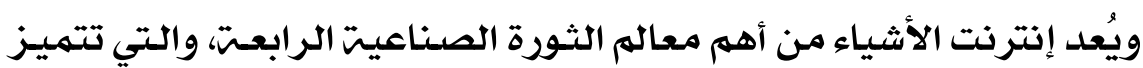

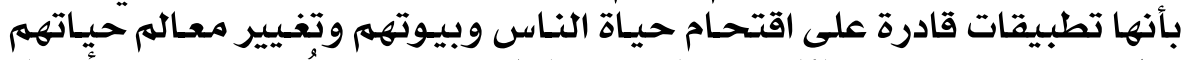

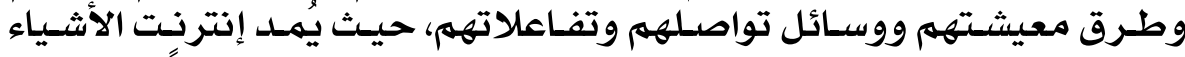

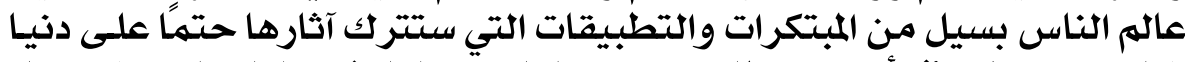

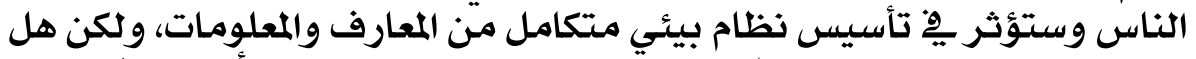

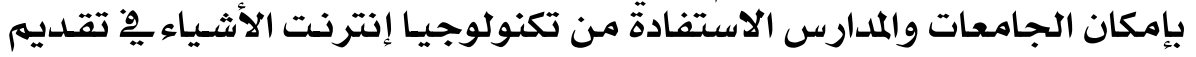

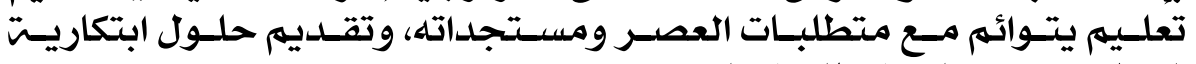

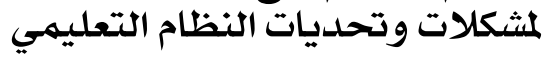

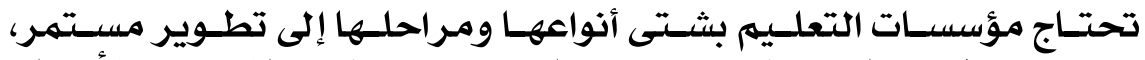

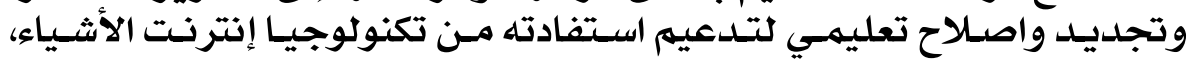

12- Harrington, J. (2018). On the usefulness of "value" in the drfinition of creativity: A commentary. Creativity Research Journal ‘30(1),P.119.

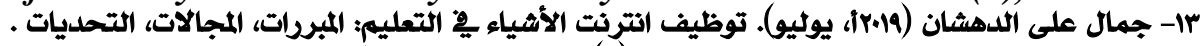

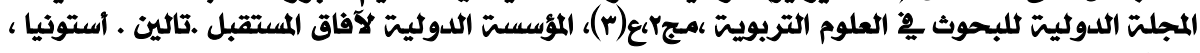




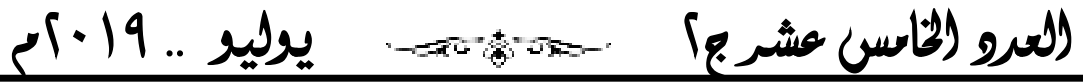

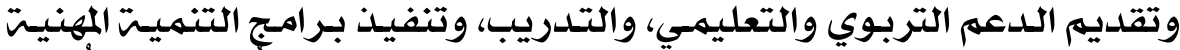

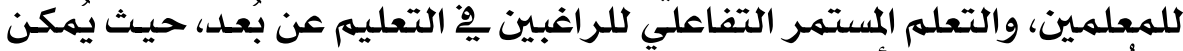

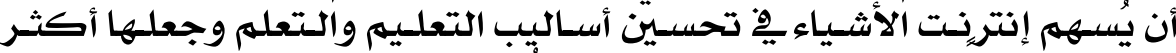

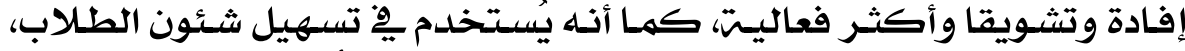

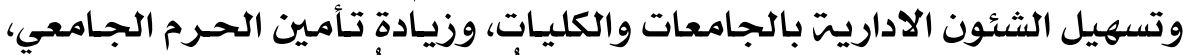

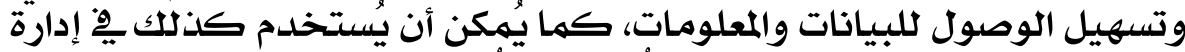

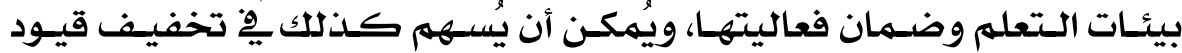

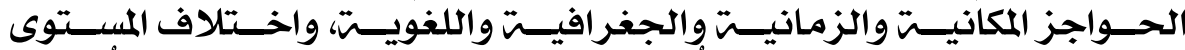

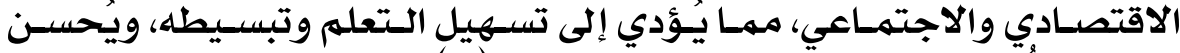

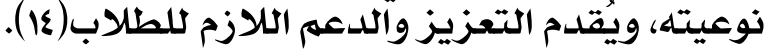

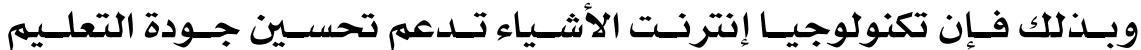

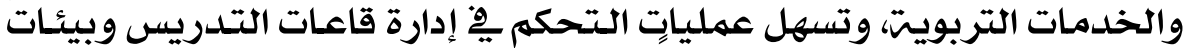

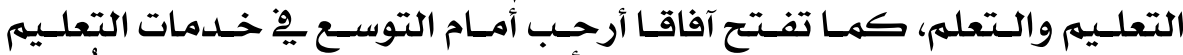

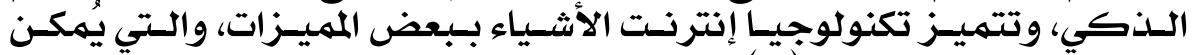

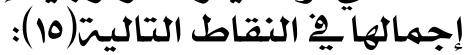

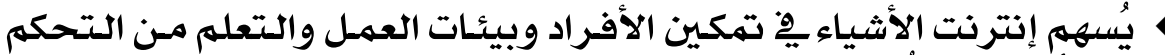

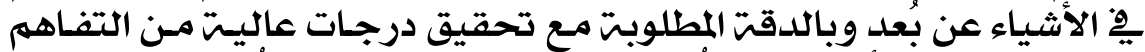

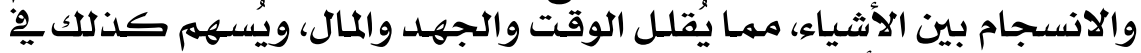

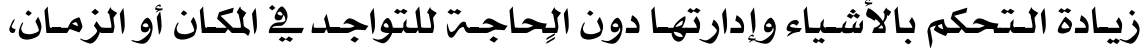

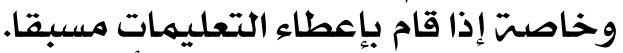

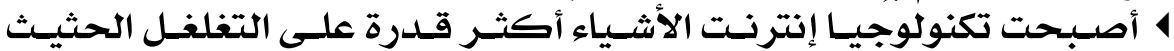

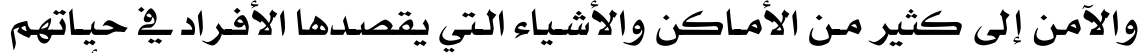

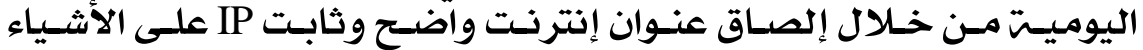

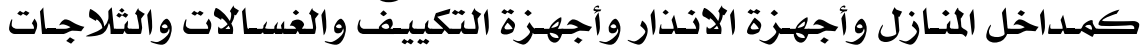

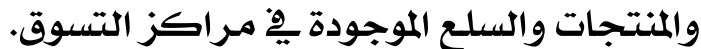

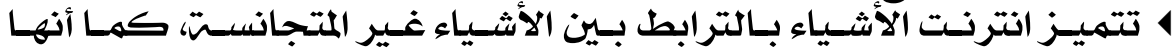

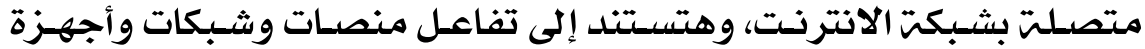

14- Siko, J. P. ‘\& Hess, A. N. (2014). Win-win professional development: Providing meaningful professional development while meeting the needs of all stakeholders.TechTrends, 58 (6),P.93. doi:http://dx.doi.org/ 10.1007/s 11528-014-0809-7

15- For more details review: Salazar, C.; Patel, K; Patel, S.(2016,May). Internet of Things -IOT: Definition, Characteristics, Architecture, Enabling Technologies, Application \& Future Challenges. International Journal of Engineering Science and Computing. Vol.6, No.(5),P. 6125.

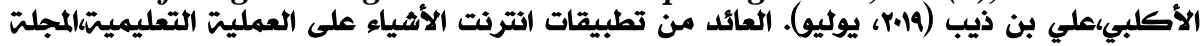

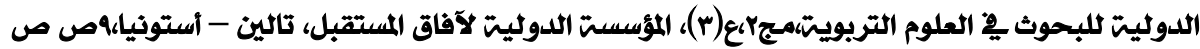


(العرو (الماسم) عشرج) .

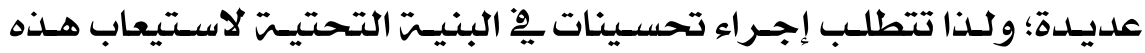
التكنولوجيا.

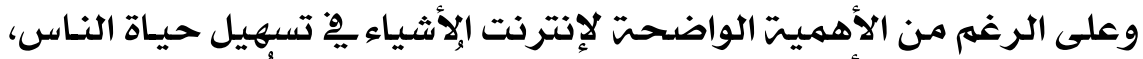

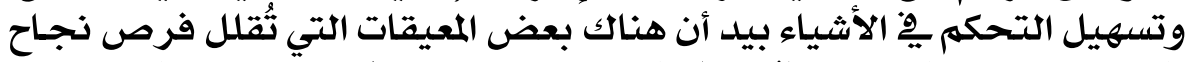

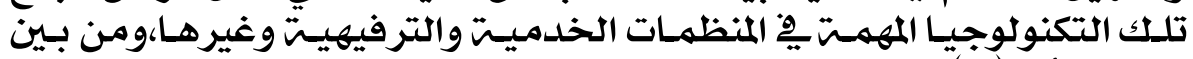
تلكك ما يأتي (17):

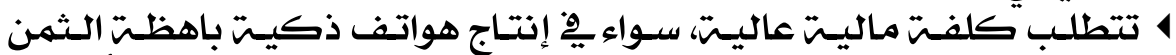

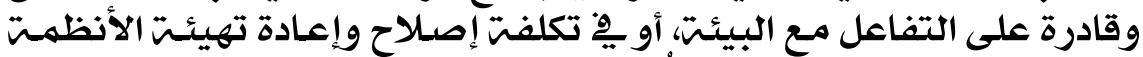

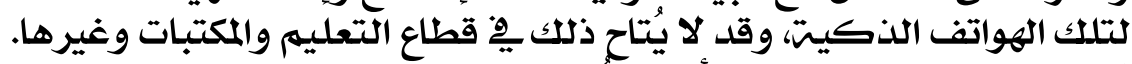

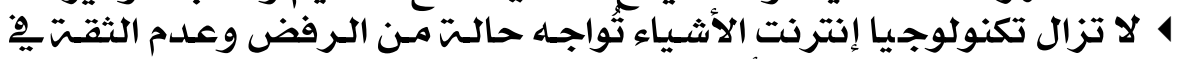

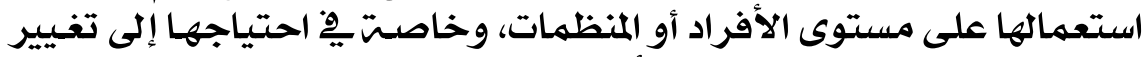

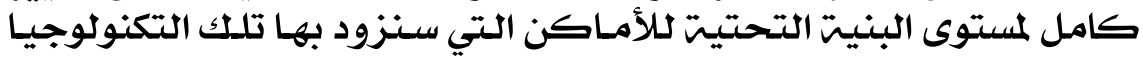
المتقدمت.

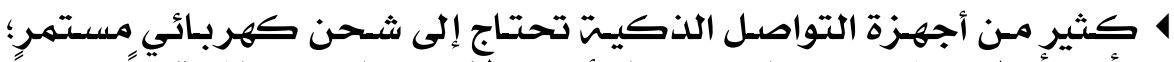

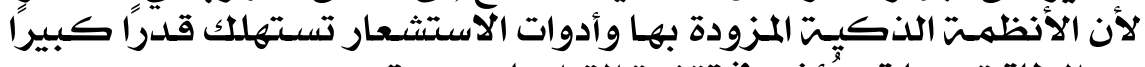

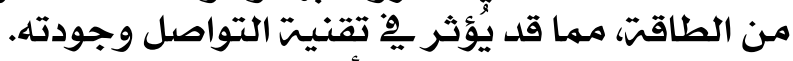

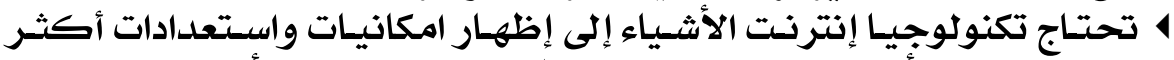

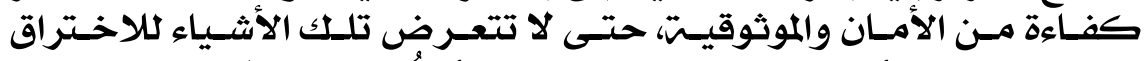

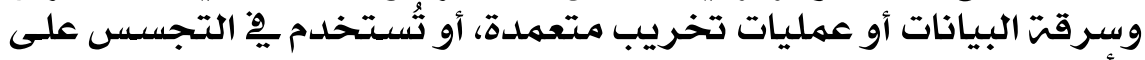

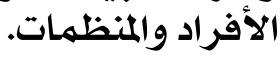

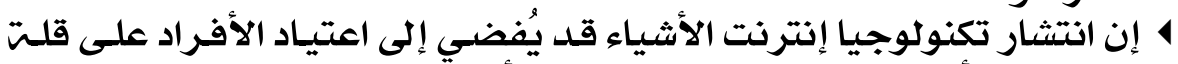

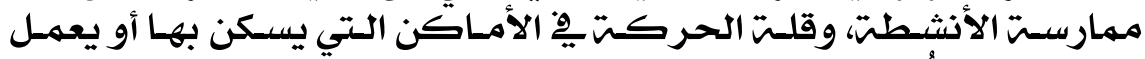

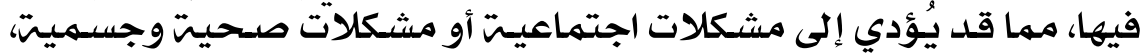

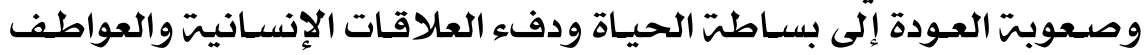
البشريتّا

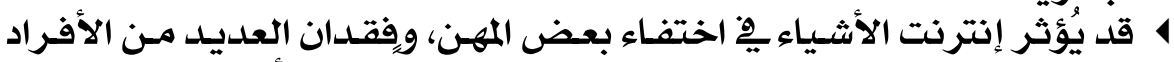

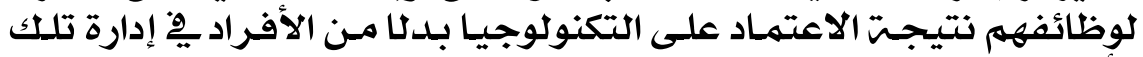
الأشياء والتحكمم فيها.

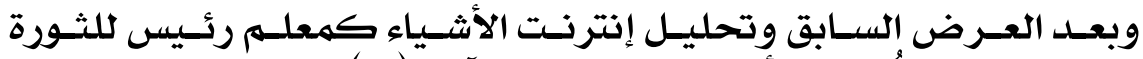
الصناعيت الرابعت يُمكن التأشير على النقلى النقاط الآتيتة(IV):

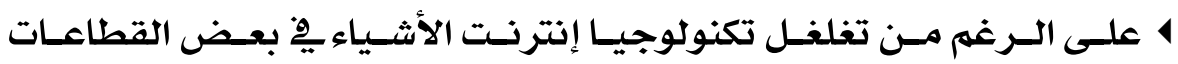

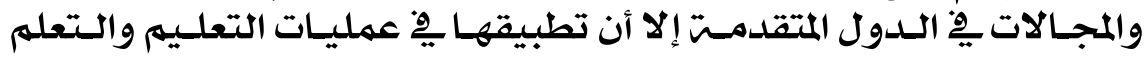

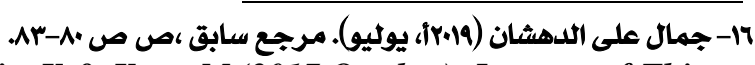

17- Hollier, S ; Mcrae, L; Ellis, K \& Kent ,M.(2017,October). Internet of Things (IOT) Education: Implications for Students with Disabilities. Curtin Learning and Teaching Innovation Grant, Curtin University of Technology, Australia, P.P.3435 


\section{(العرو (الخاس) عشرج) .}

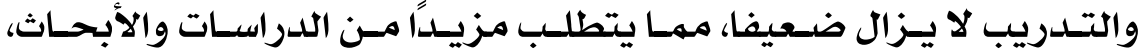

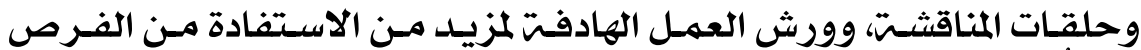

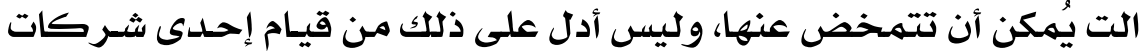

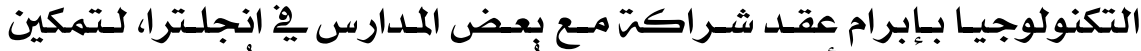

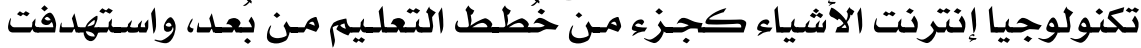

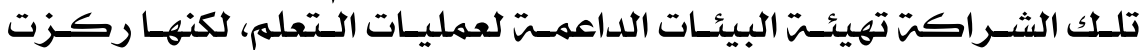

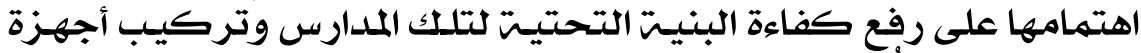

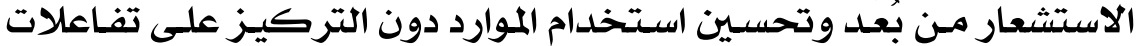

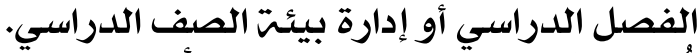

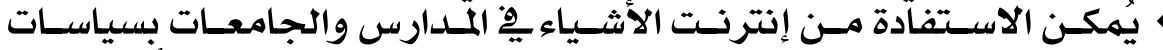

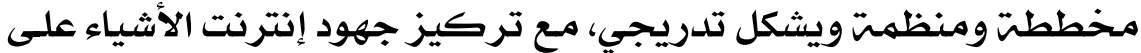

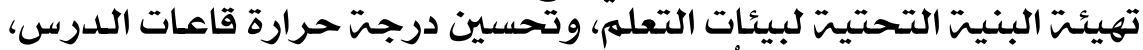

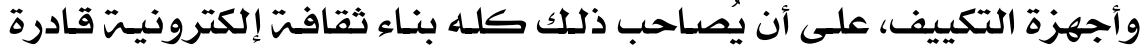

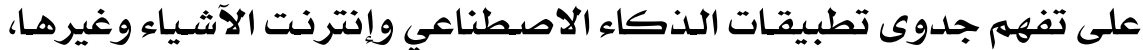

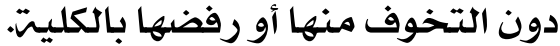

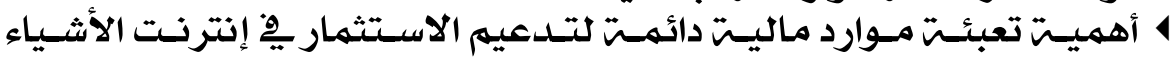

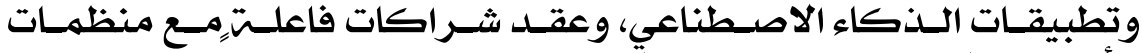

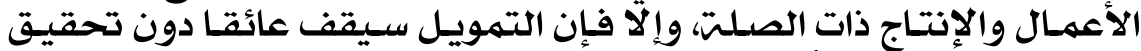

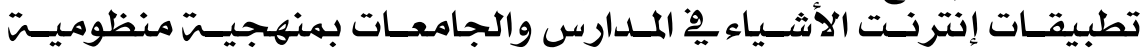
صحيحت.

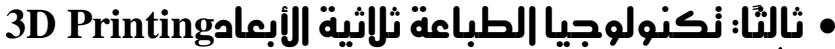

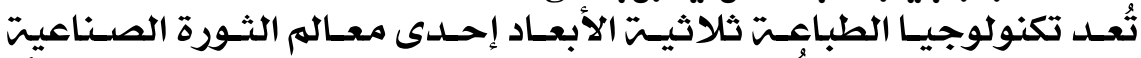

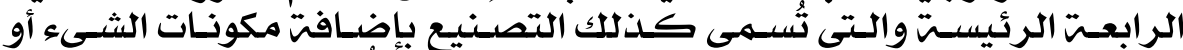

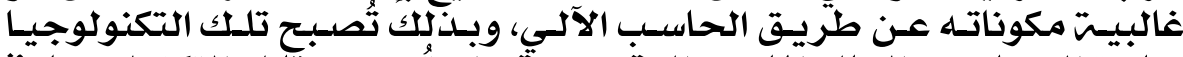

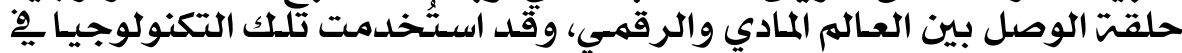

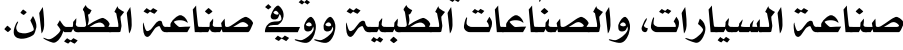

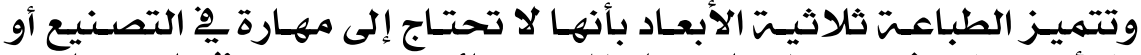

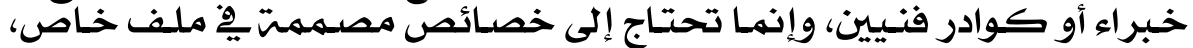

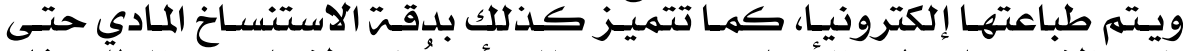

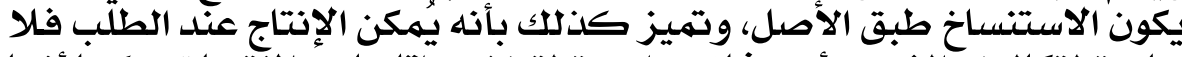

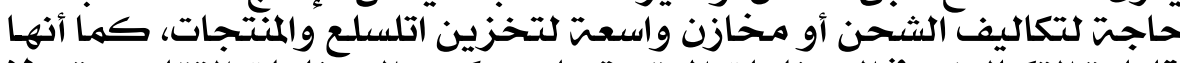

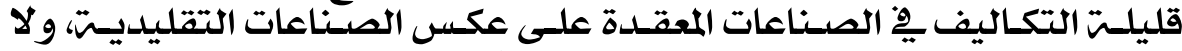

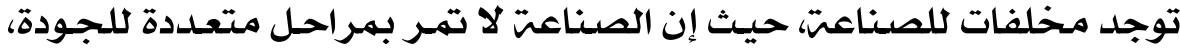

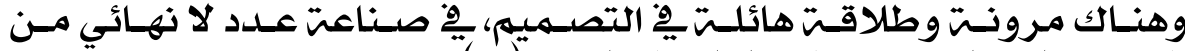

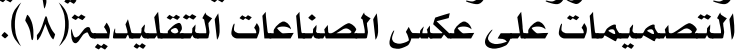

18- Ford, S.; Minshall ,T.( 2016, October). 3D Printing in Education : a Literature Review. Researcg Gate, para 5. At: https://dfab.it/3DPin $\underline{\text { Teachingpreprint }}$ 


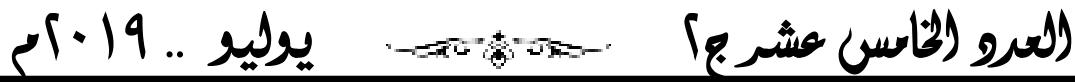

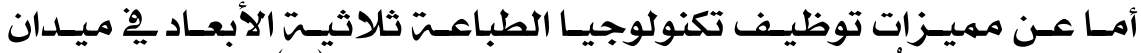

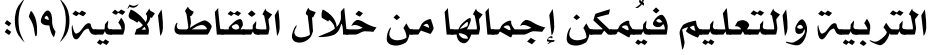

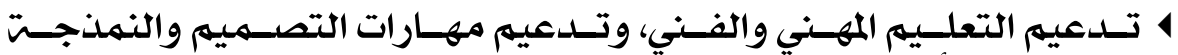

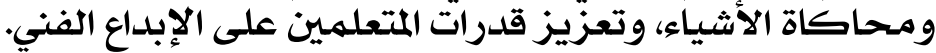

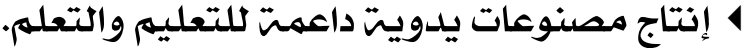

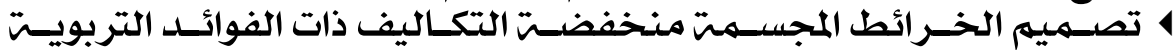

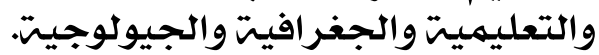

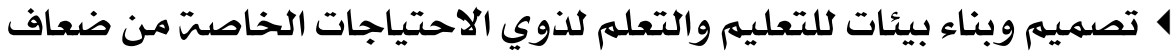

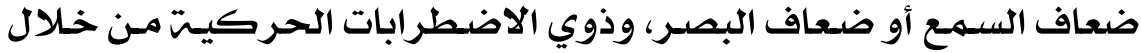

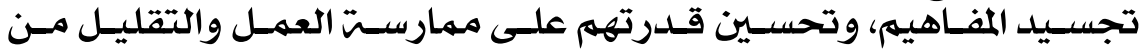
الاغتراب الاجتماعي، وتسهيل التعلهم النشط والتعاوني.

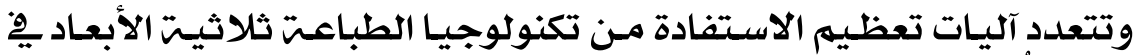

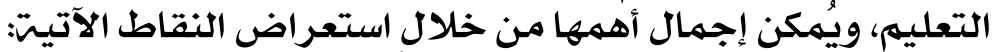

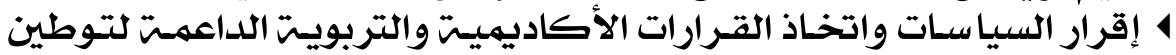

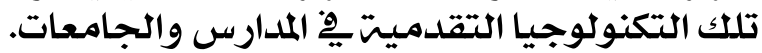

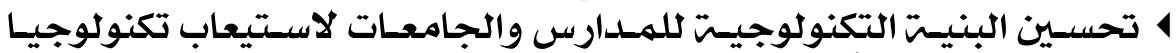
الطباعت ثلاثيت الأبعاد.

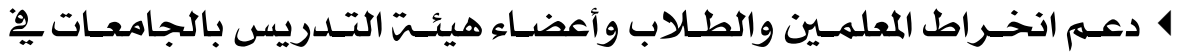

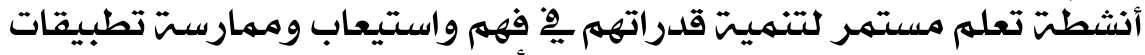

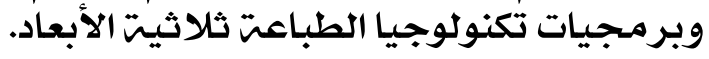

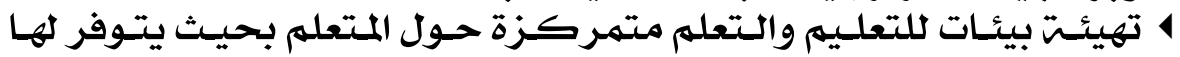

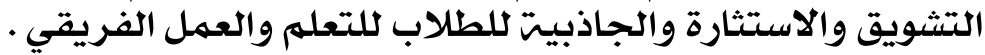

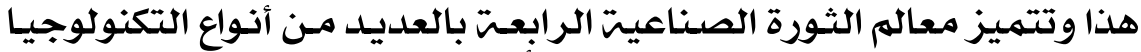

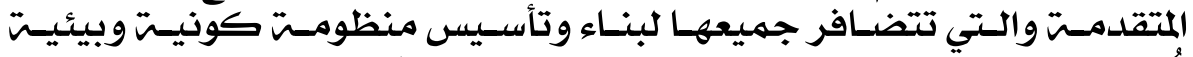

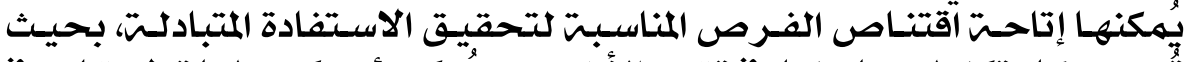

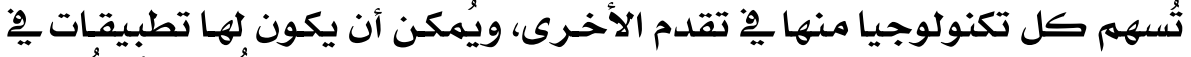

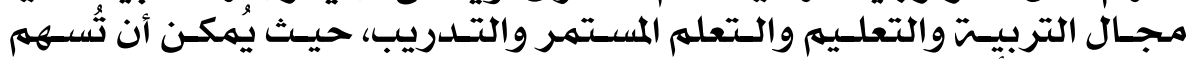

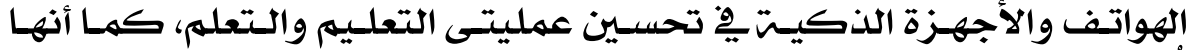

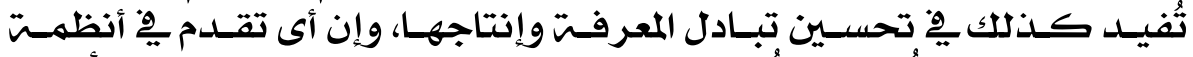

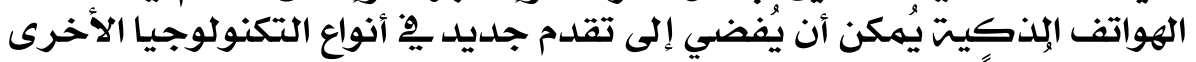

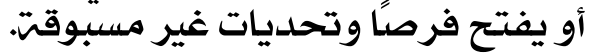

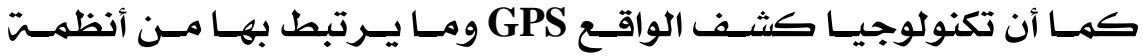

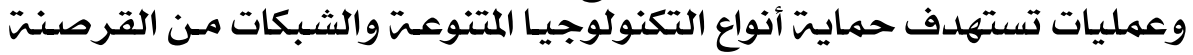

19- Arvanitidi, E.; Drosos, C.; Theocharis, E.; Papoutsidakis, M.(2019, December). 3D Printing and Education . International Journal of Computer Applications(0975-8887), Vol.177, No.24,P.57. 


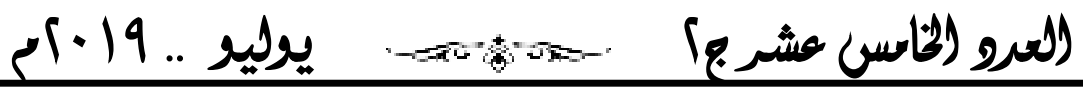

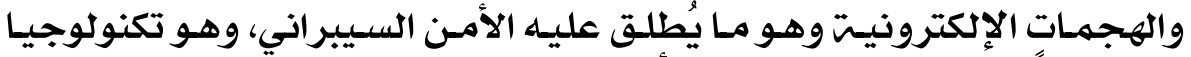

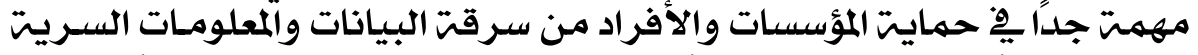

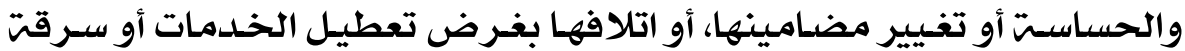
الملكيت الفكريت أو ابتزاز الضيتهايا.

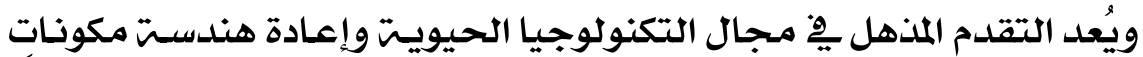

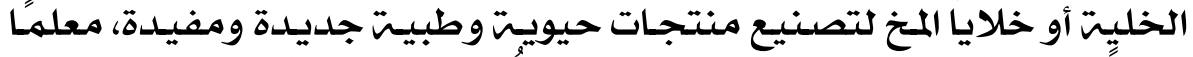

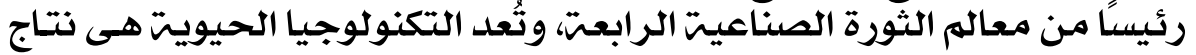

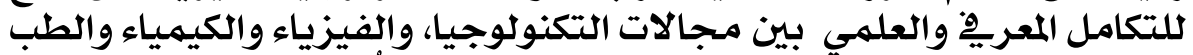

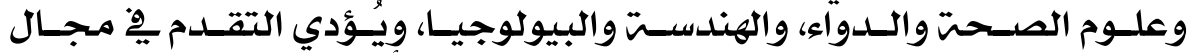

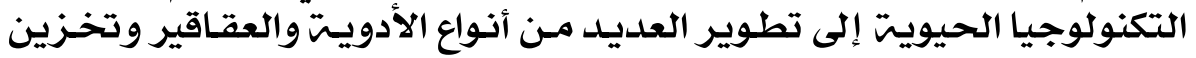

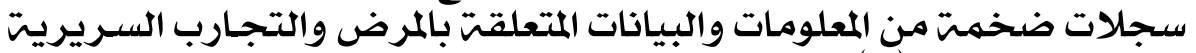

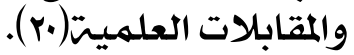

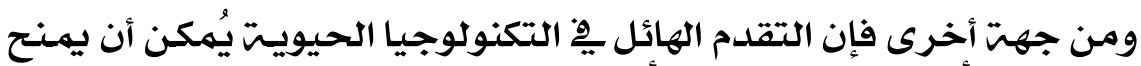

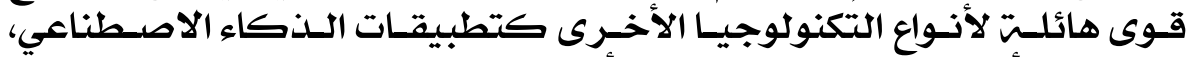

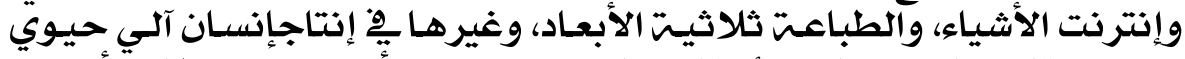

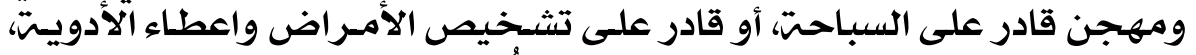

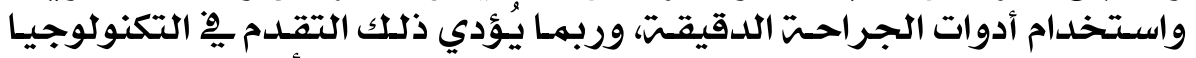

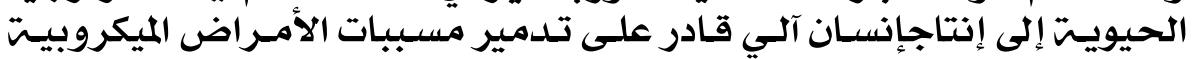
والفيروسيت كمر إلى إنتانسان الكورونا وغيره.

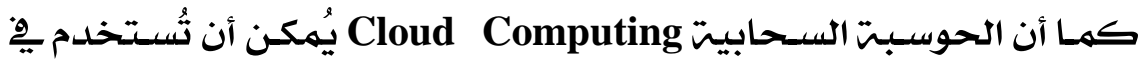

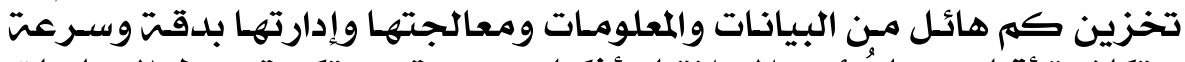

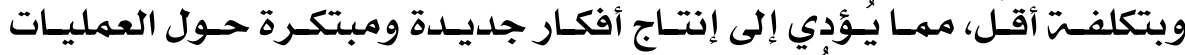

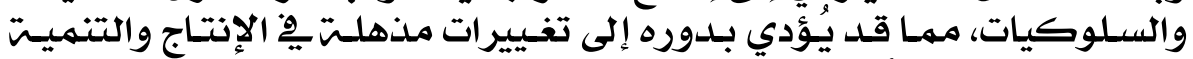

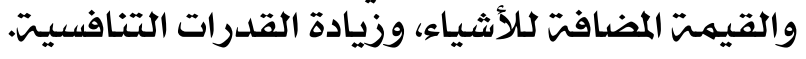

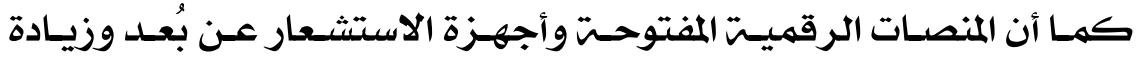

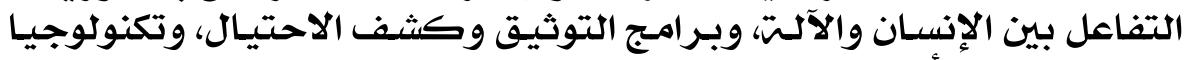

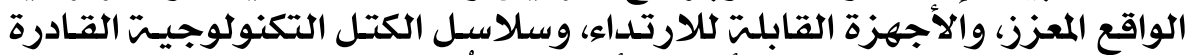

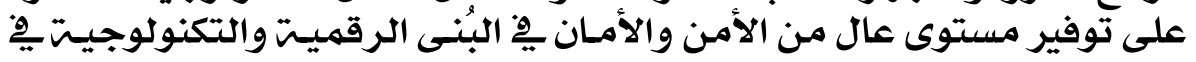

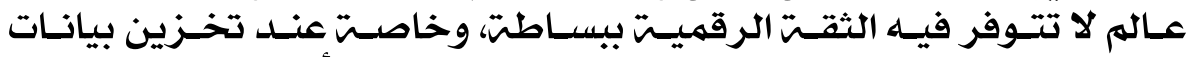

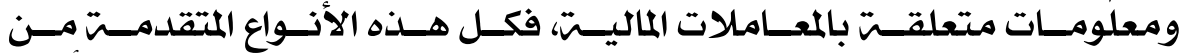

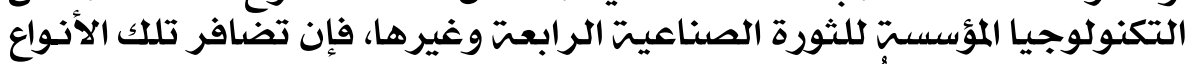

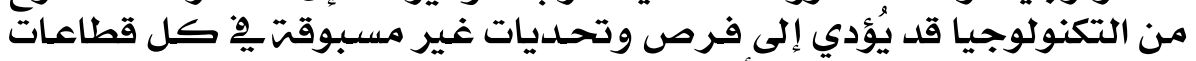

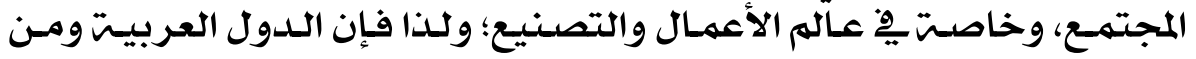

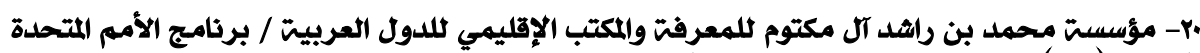

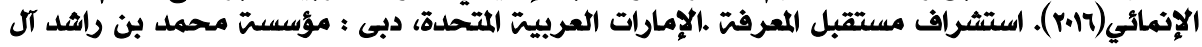




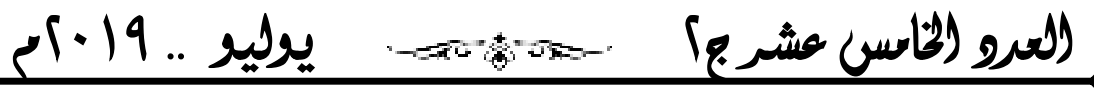

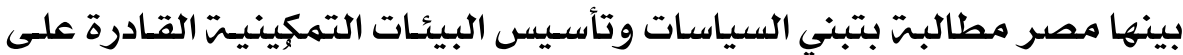

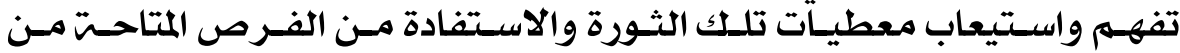

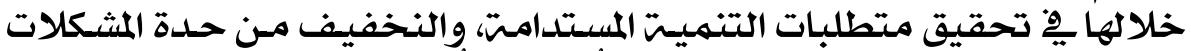

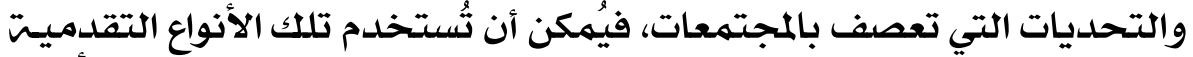

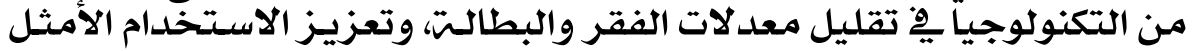

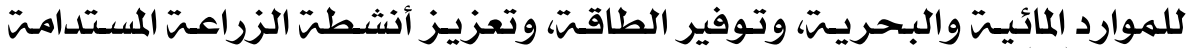
وغيرها(Y) (بارد). • المحور الثـانيه: نـم|عيانه الثـورة الصناعية الرابعة علىه منظومة النعليه الجامعم وكليانه التزبية

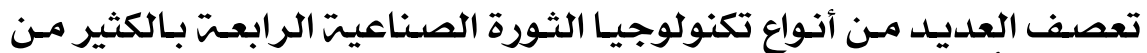

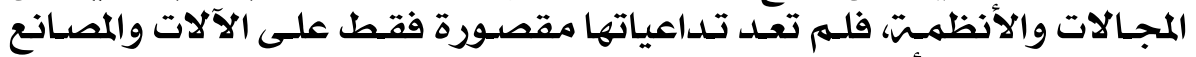

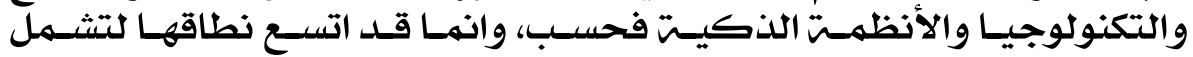

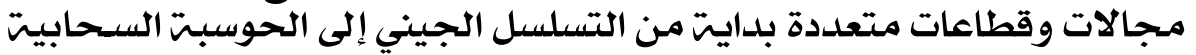

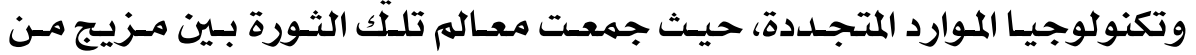

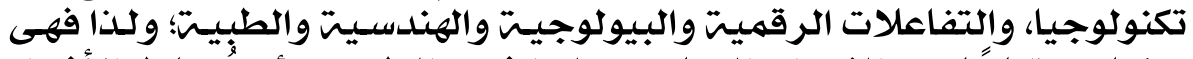

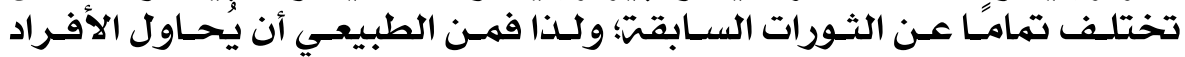

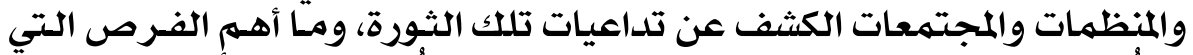

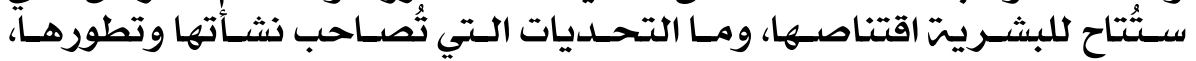

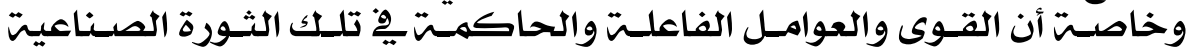

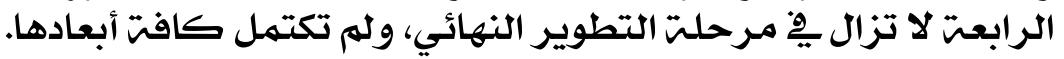

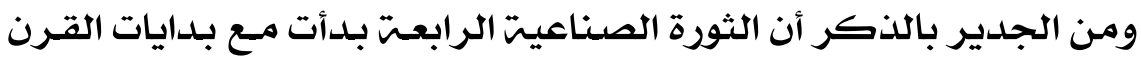

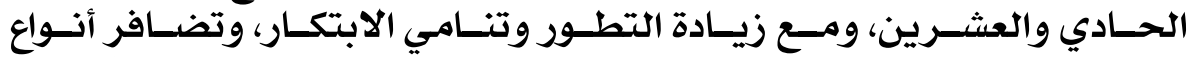

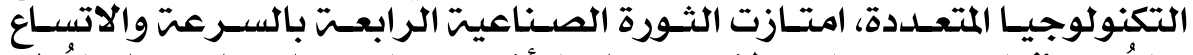

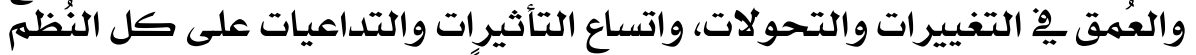

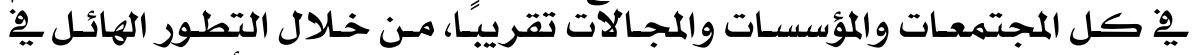

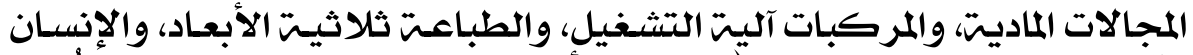

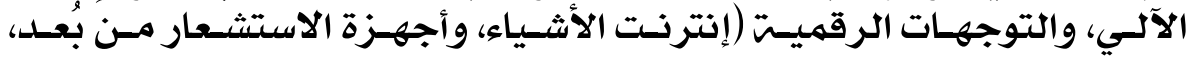

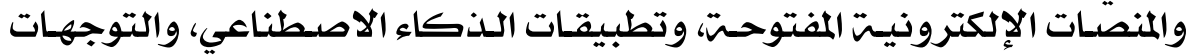

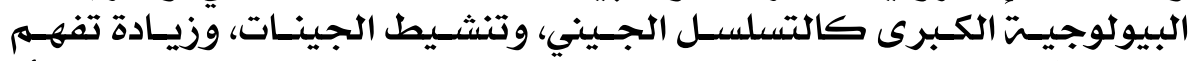

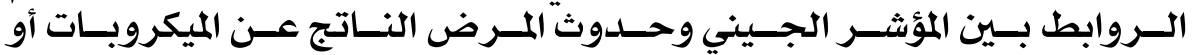

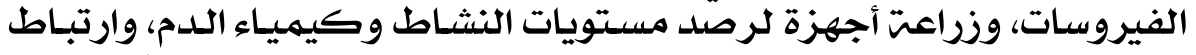

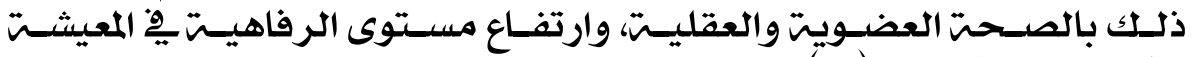

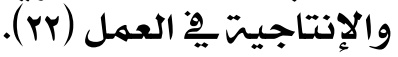

21- Jagirdar, S.(2012, July). Cloud Computing Basics. International Journal of Advanced Research in Computer and Communication Engineering, Vol.1, Issue $5,344$.

22- Klaus Schwab,2016,Opcit, p.p26-27. 
(العرو (لخاس) عشرج) .

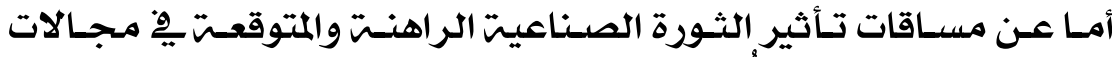

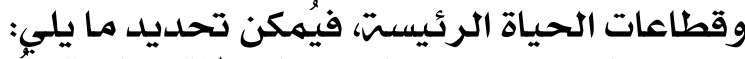

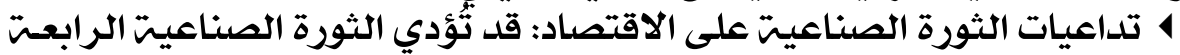

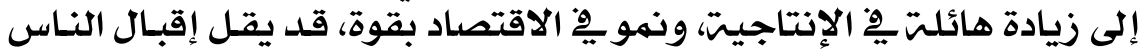

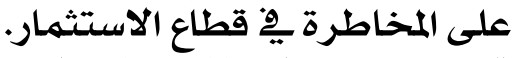

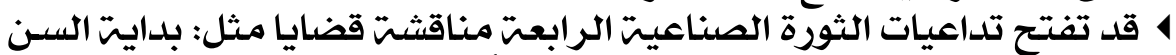

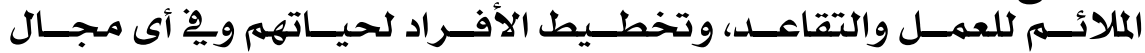

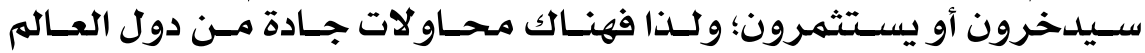

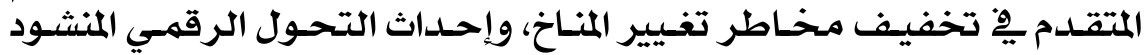

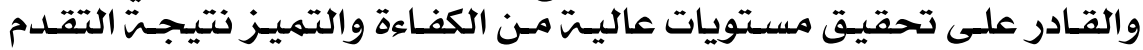
التتكنولوجي الهادئل.

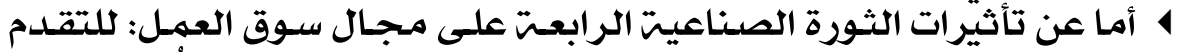

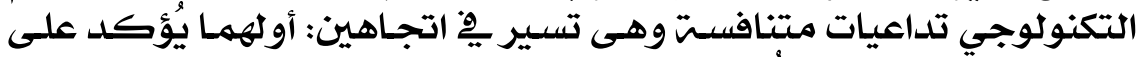

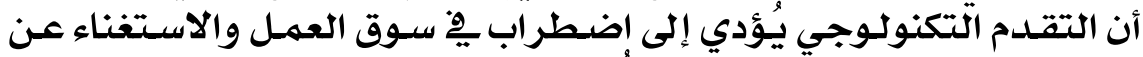

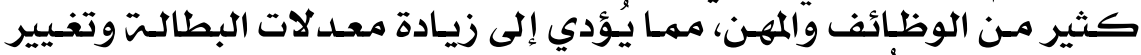

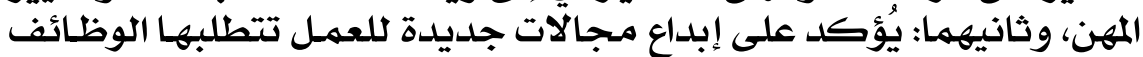

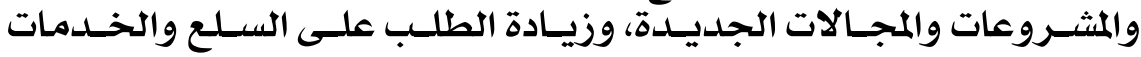
الجديلدة والمتججددة.

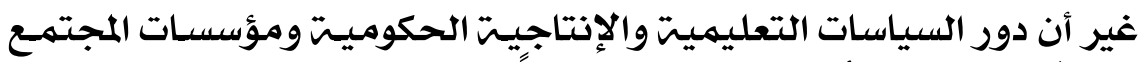

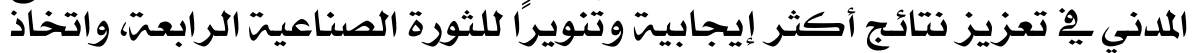

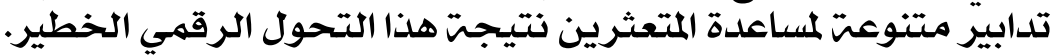

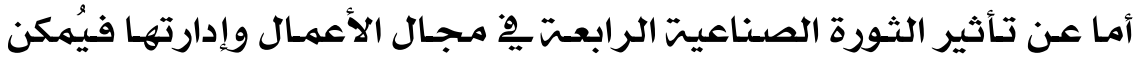

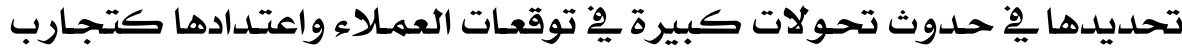

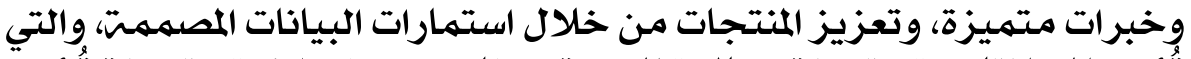

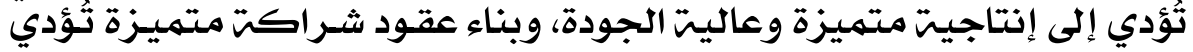

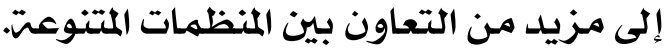

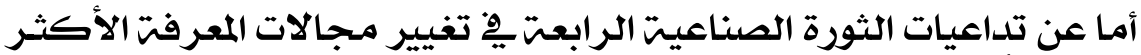

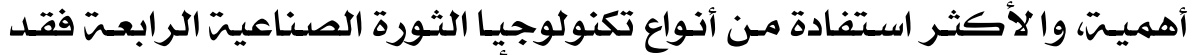

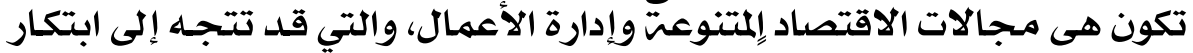

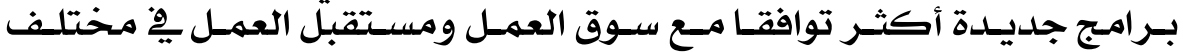
القطاعات.

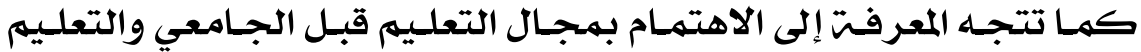

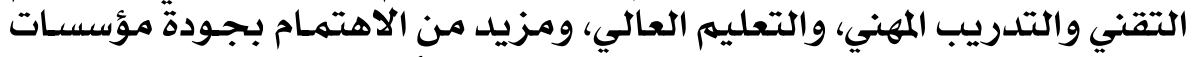

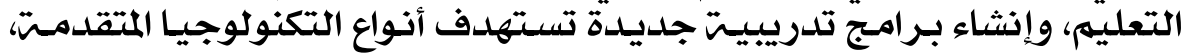

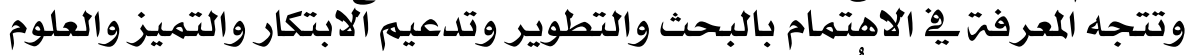

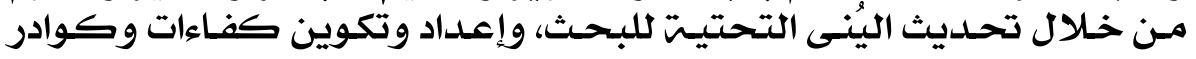




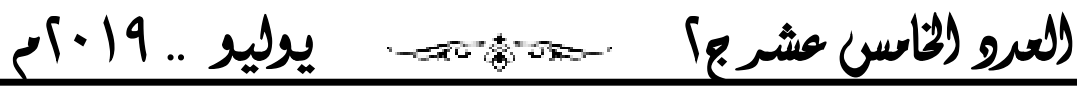

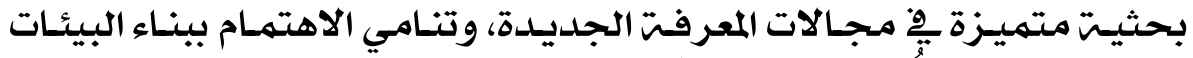

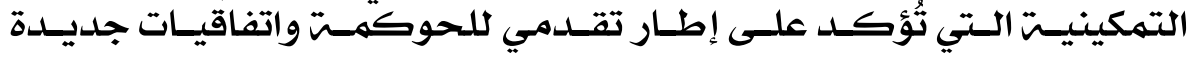

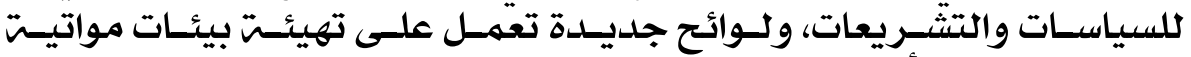

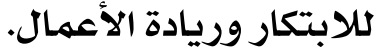

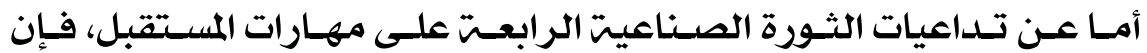

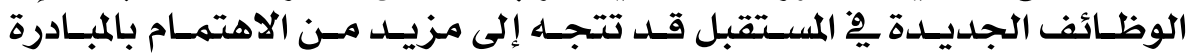

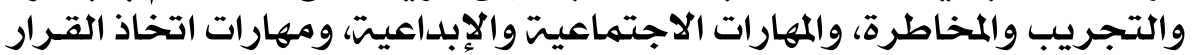

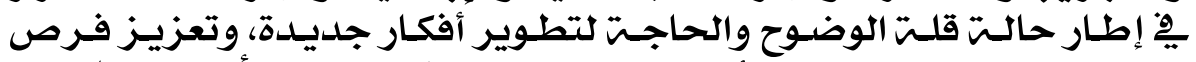

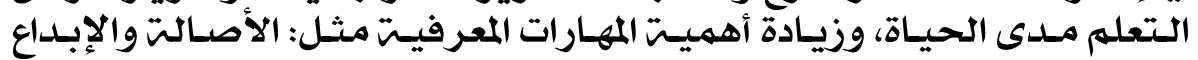

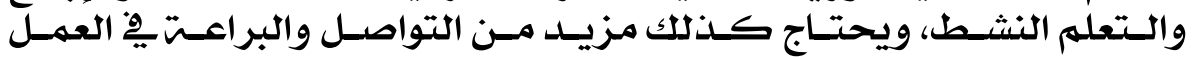

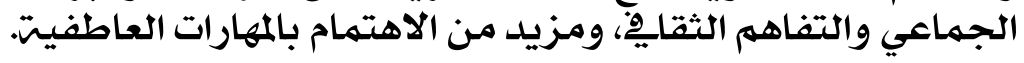

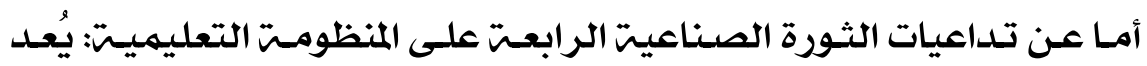

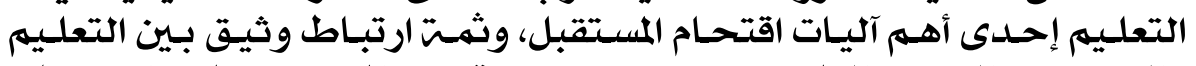

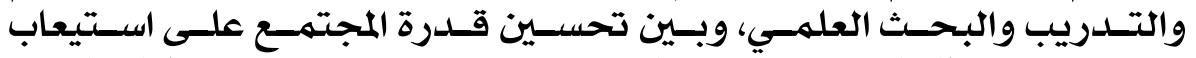

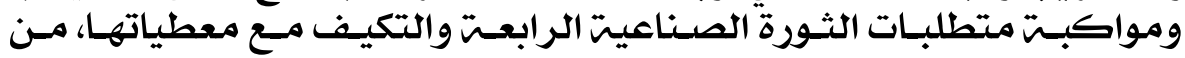

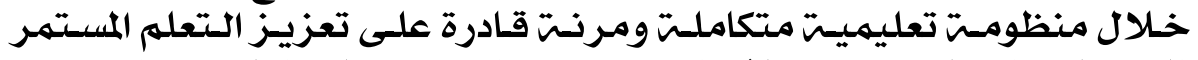

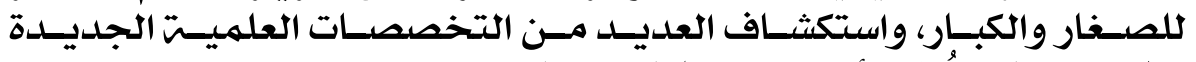

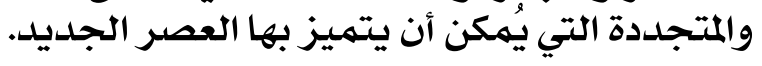

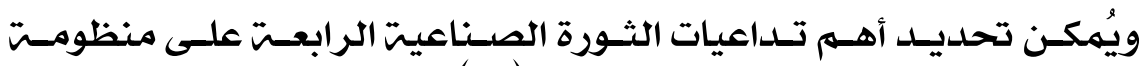

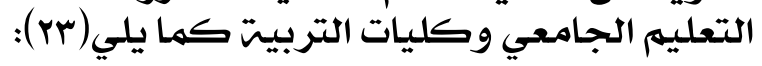

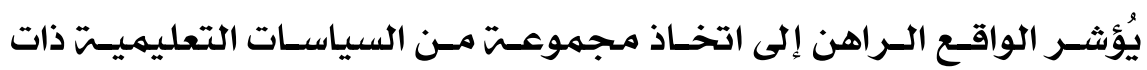

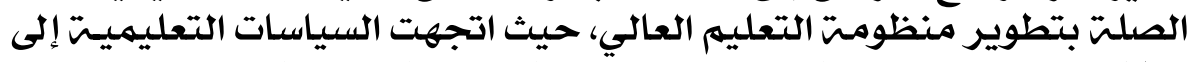

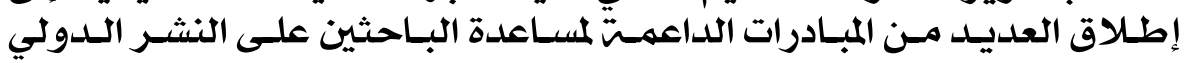

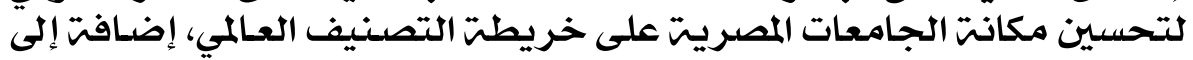

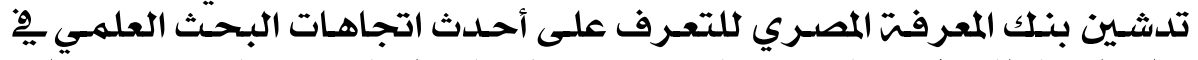

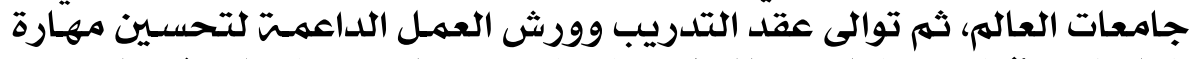

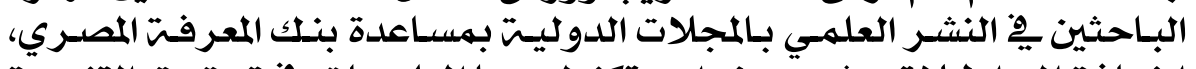

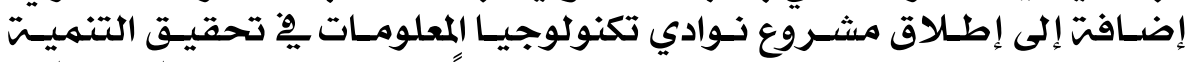

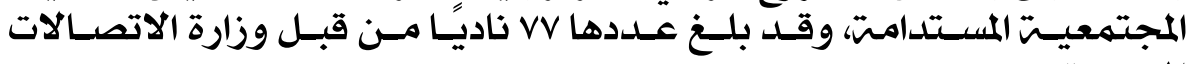
المصريت.

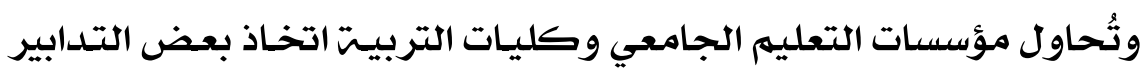

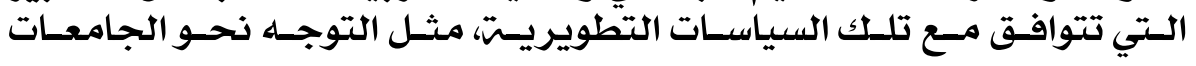

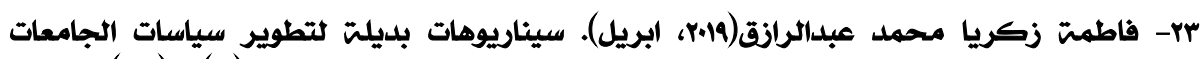

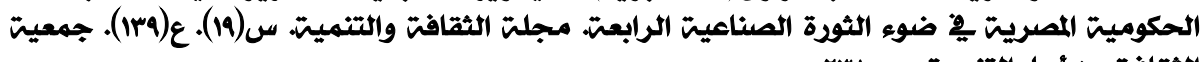




\section{(العرو (لأاسم) عشرج) .}

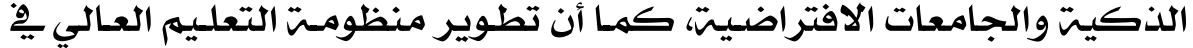

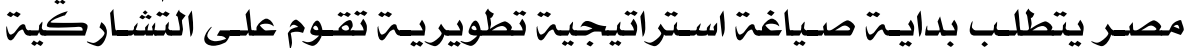

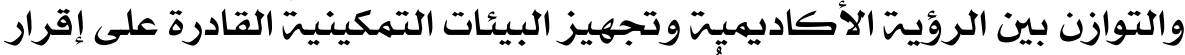

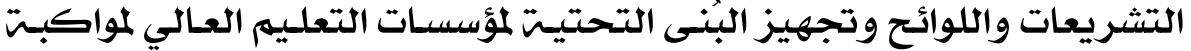

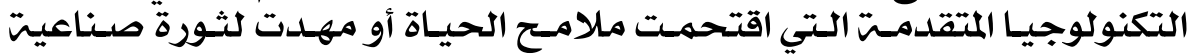
رابعت.

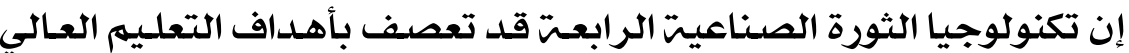

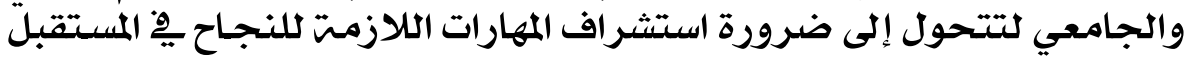

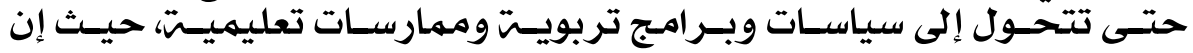

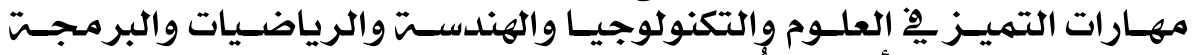

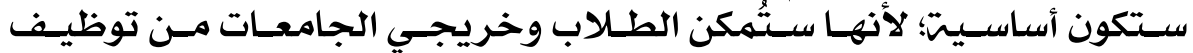

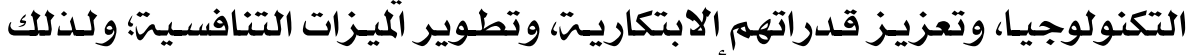

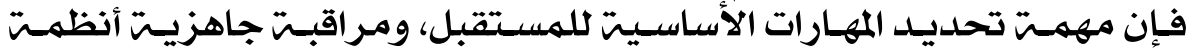

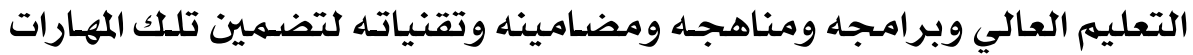

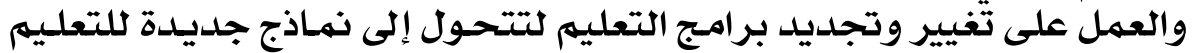

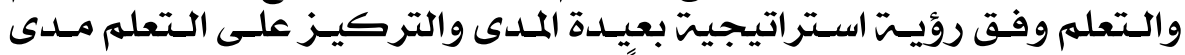

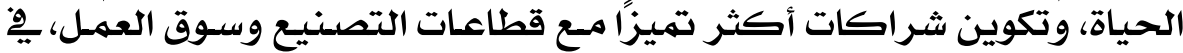

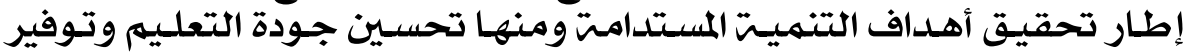

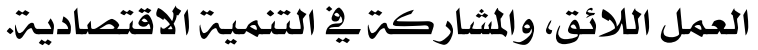

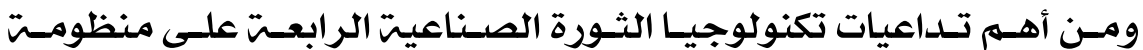

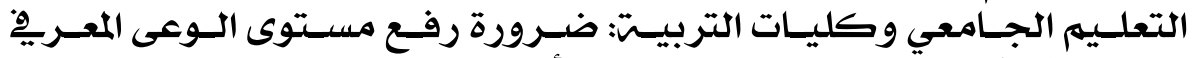

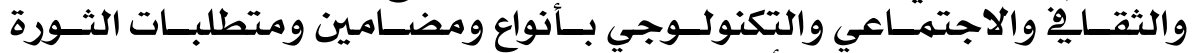

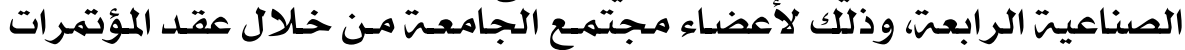

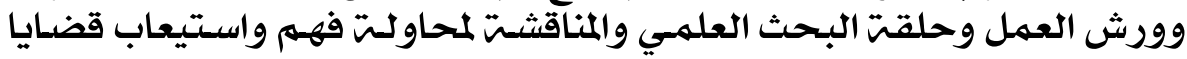

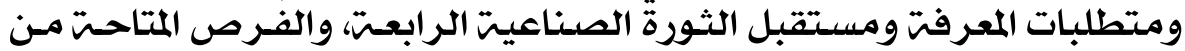

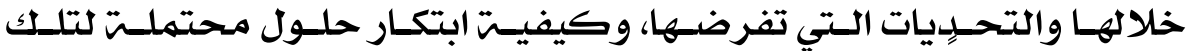

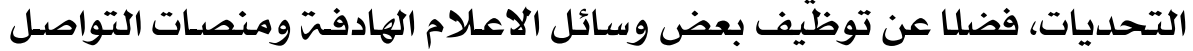

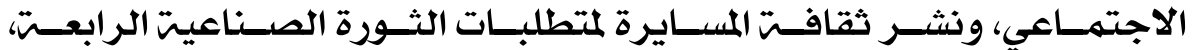

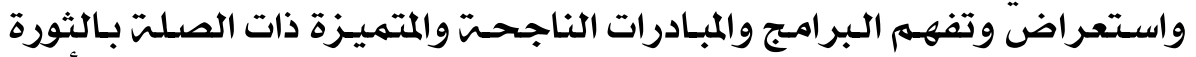

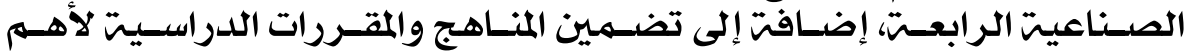

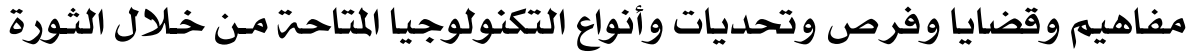
الصناعيت الرابعت.

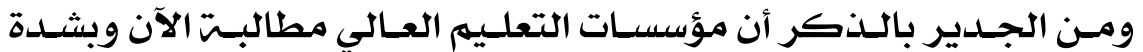

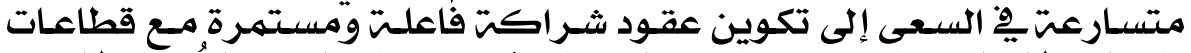

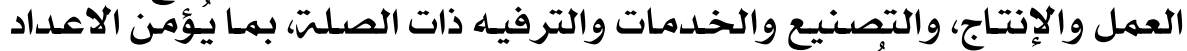

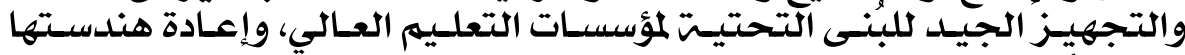

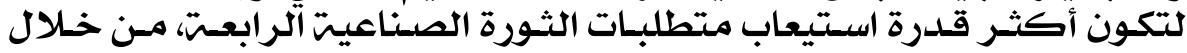




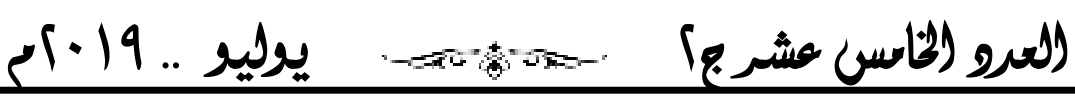

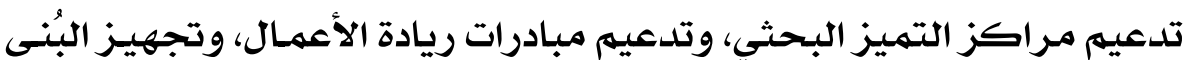

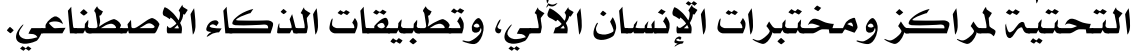

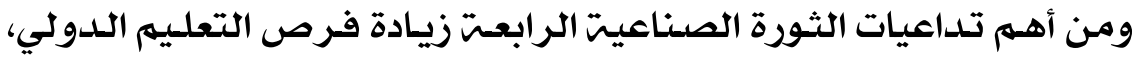

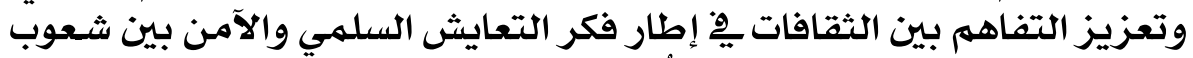

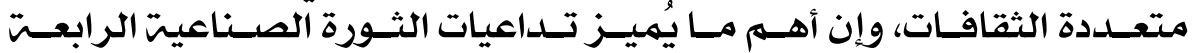

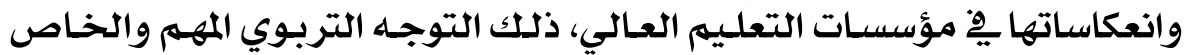

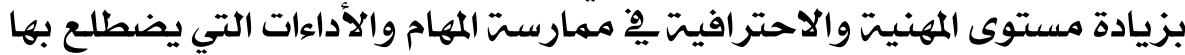

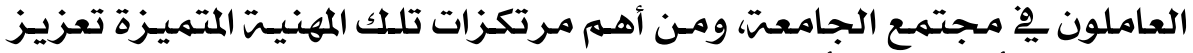

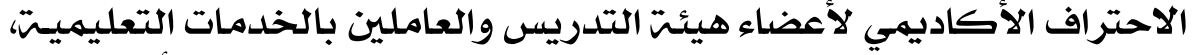

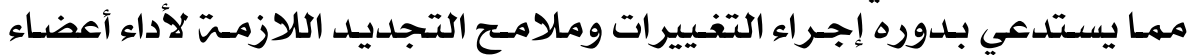

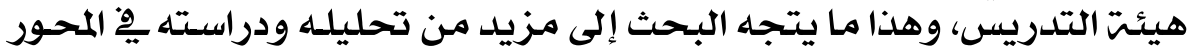
التالي.

• المحور الثالث : رؤية مقتزحة لأهم الأدوار الجديدة والهنجدمة لأنمضاء لهيئة

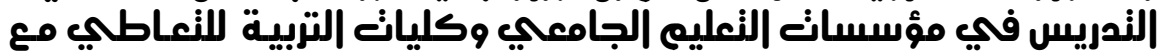

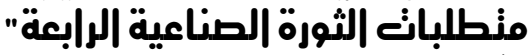

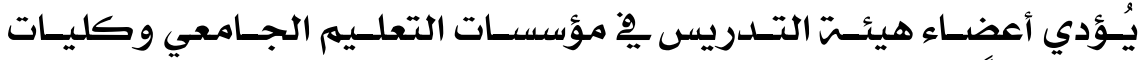

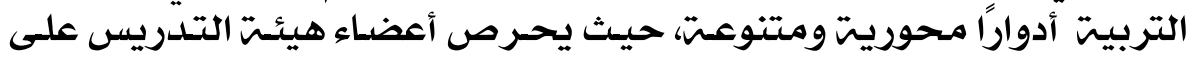

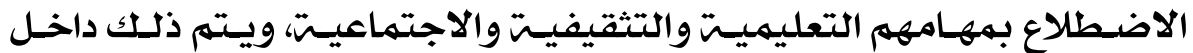

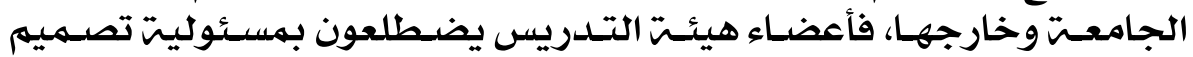

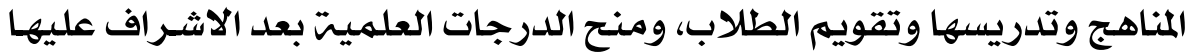

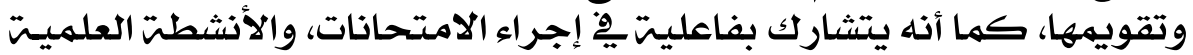

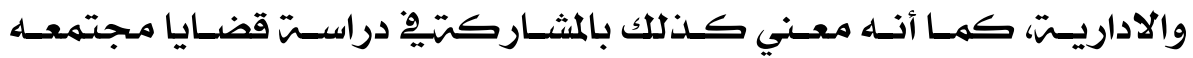

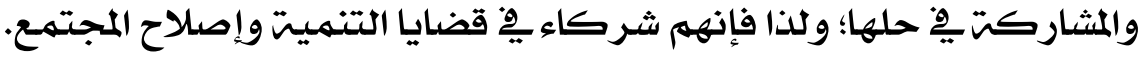

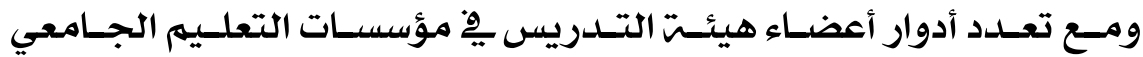

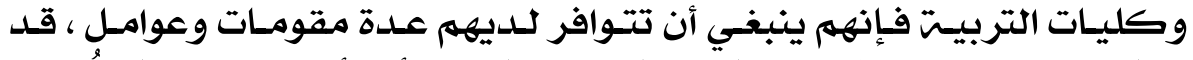

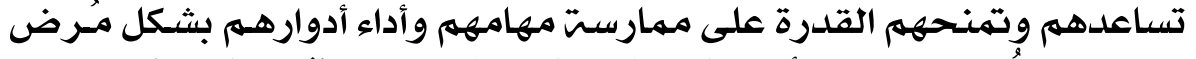

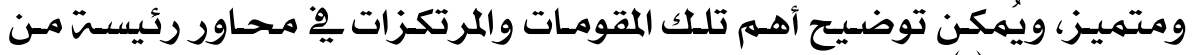
خلال الثكل (1): تونئن

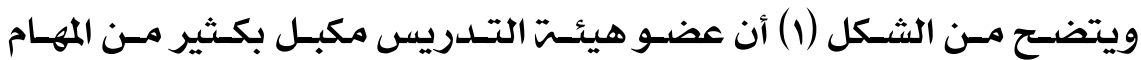

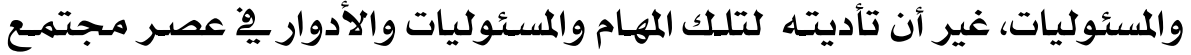

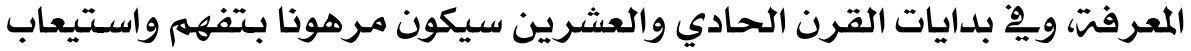

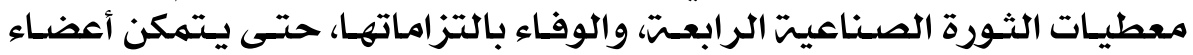

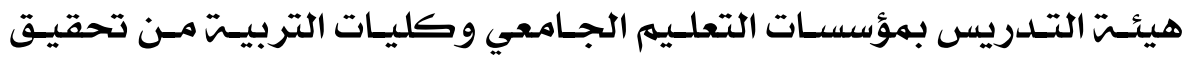

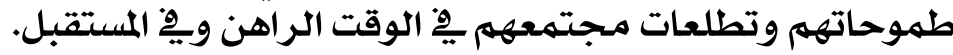




\section{(العرو (الخاسر) عشرج) .}

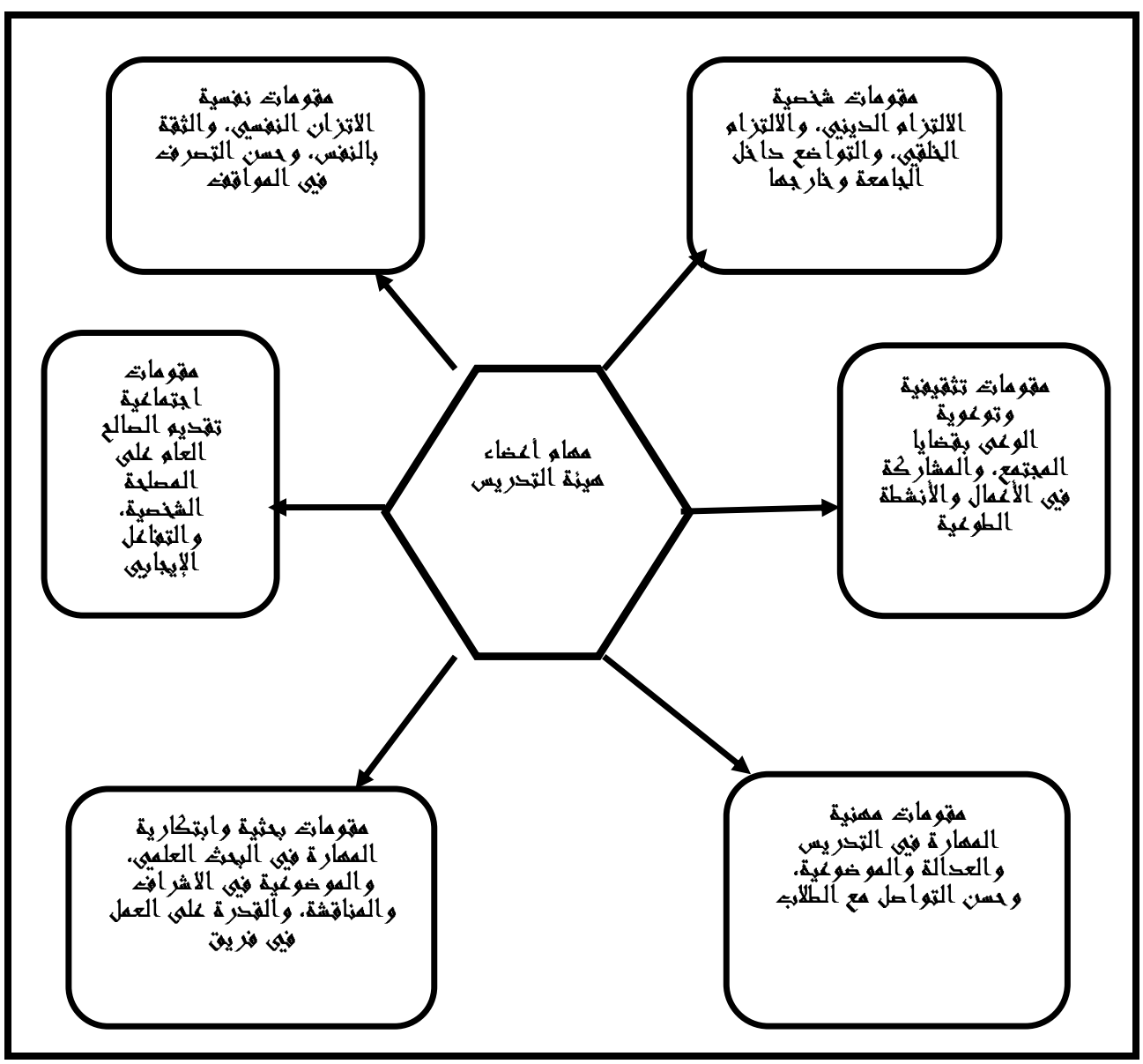

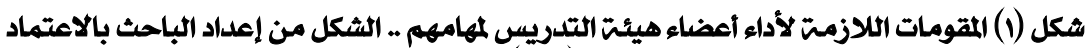

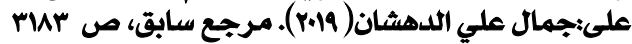

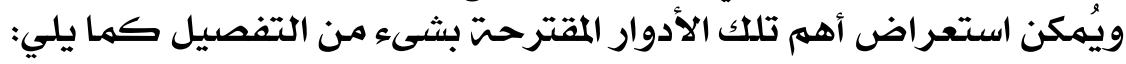

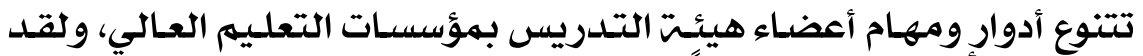

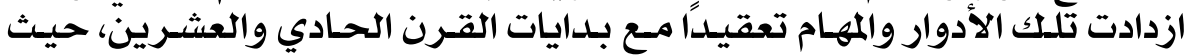

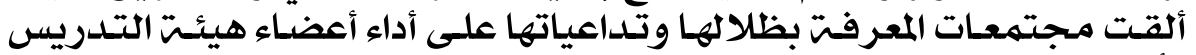

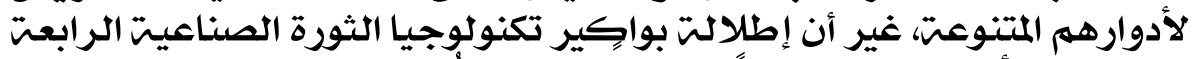

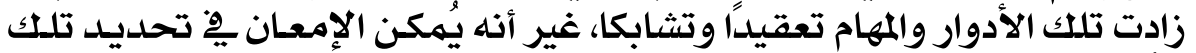

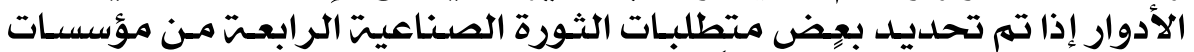

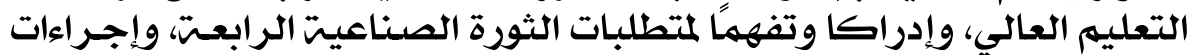

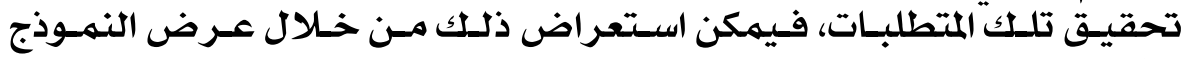


(العرو (الخاس) عشرج) .

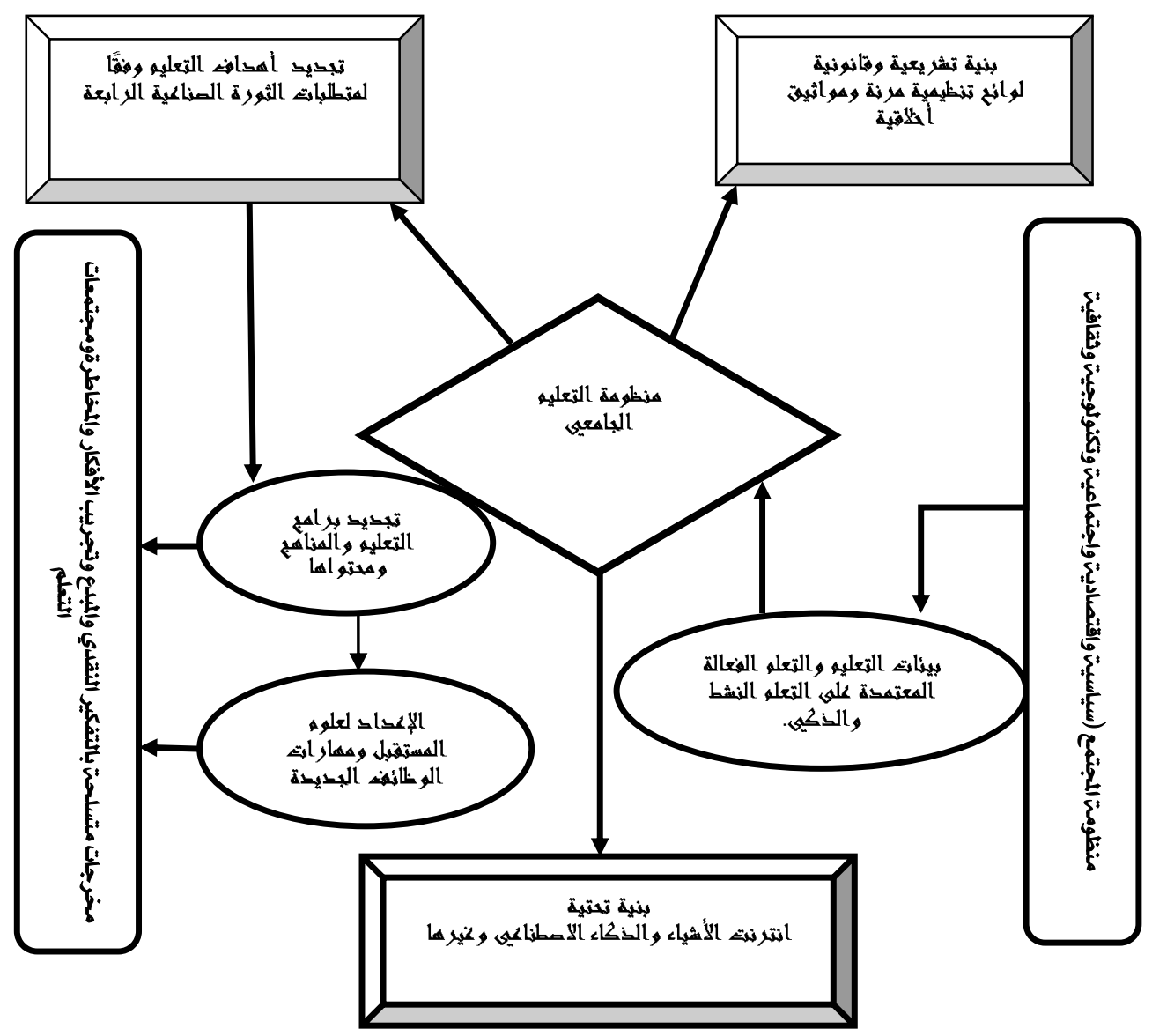

ثكل(Y) أبرز متطلبات الثورة الصناميت الرابعت من منظومت التعليم الجامعي وكليات التربيت الثكل من

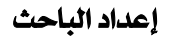

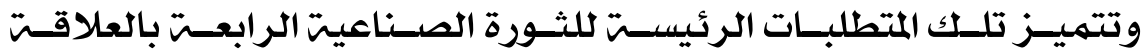

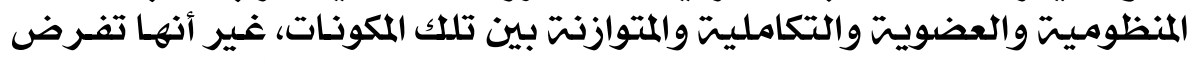

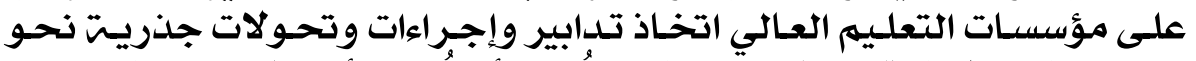

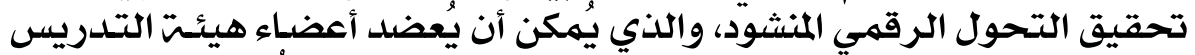

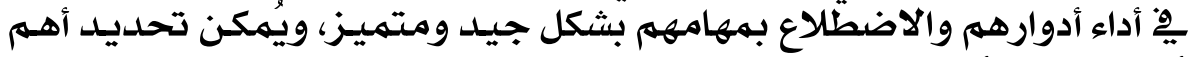

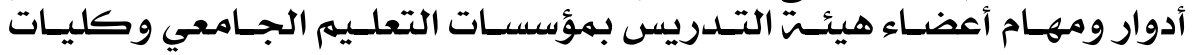

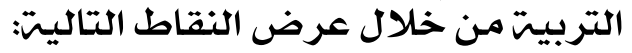

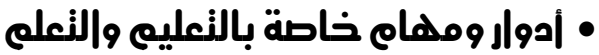

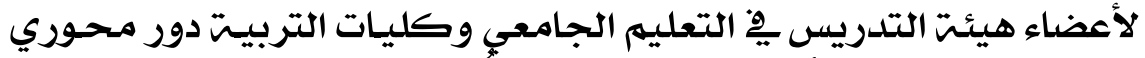

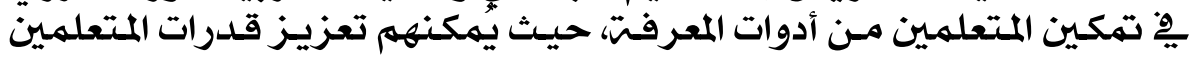




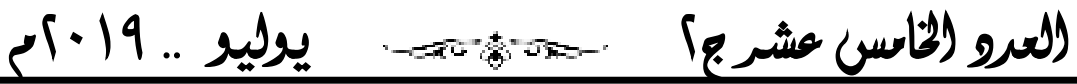

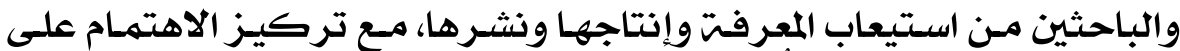

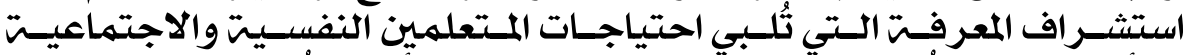

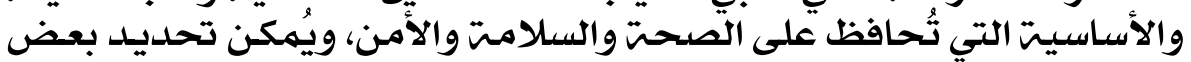

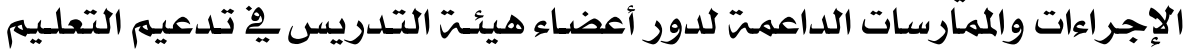

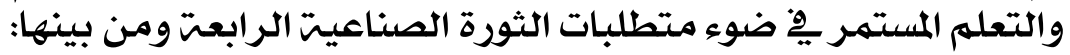

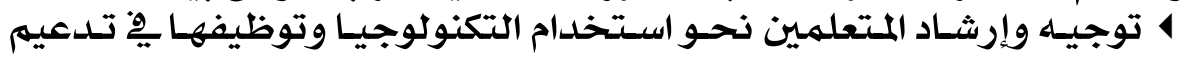
التعلهم وتسهيله وارثياد.

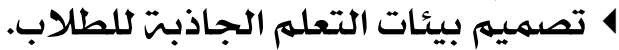

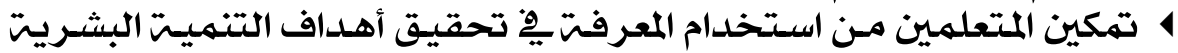

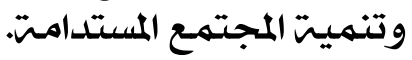

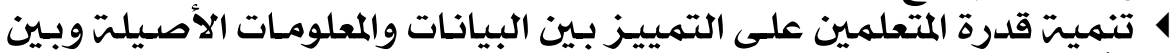

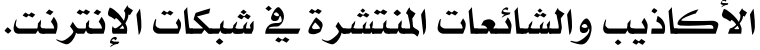

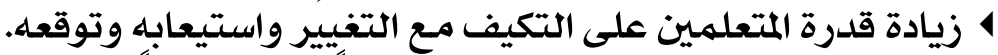

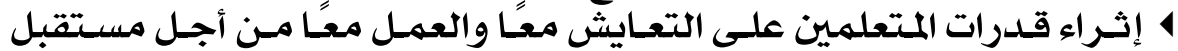

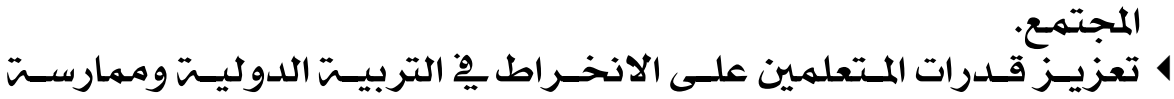

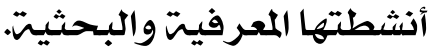

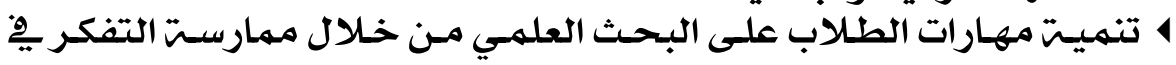

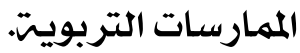

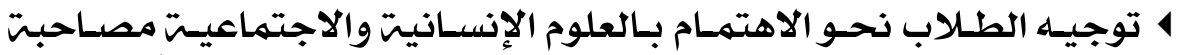

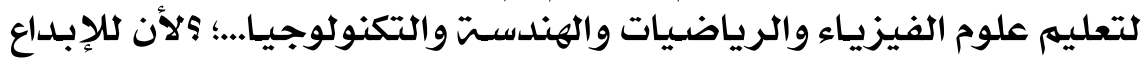

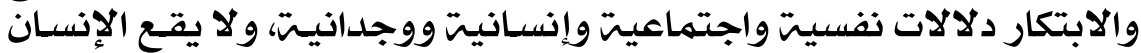

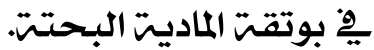

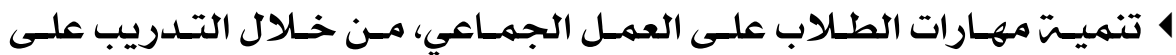

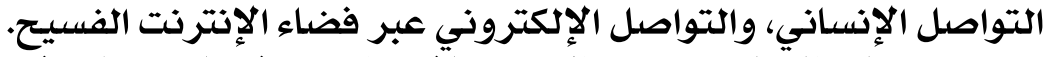

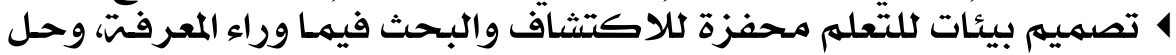

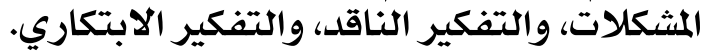

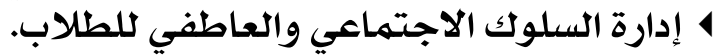

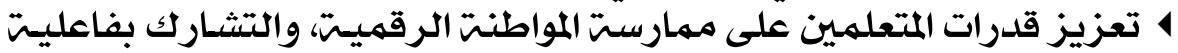

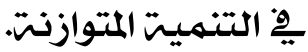

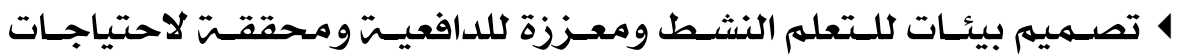

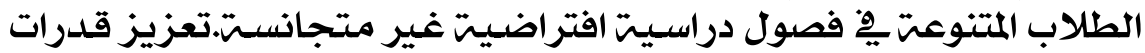

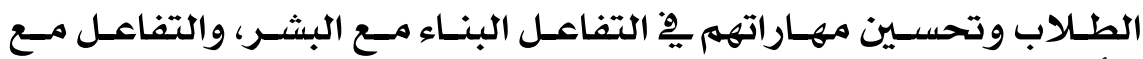

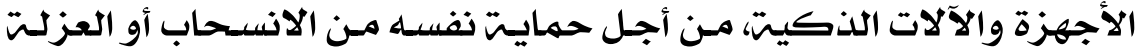
الاجتماعيت.

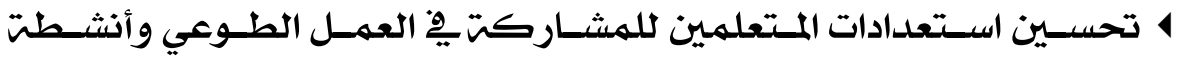




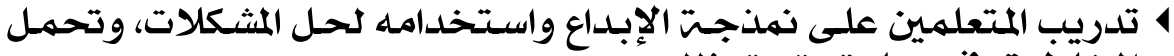

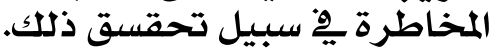

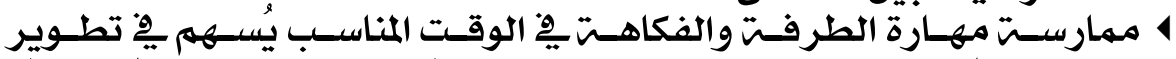

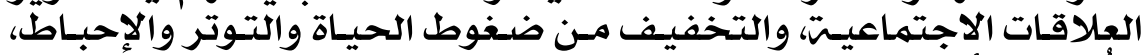

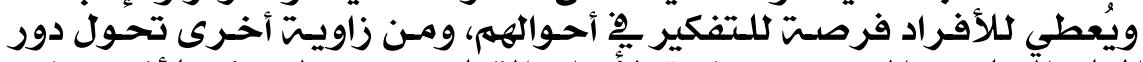

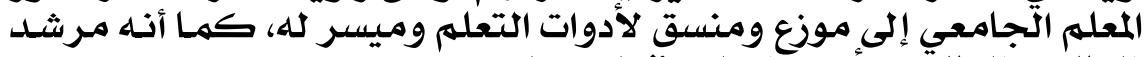

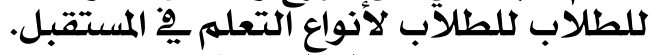

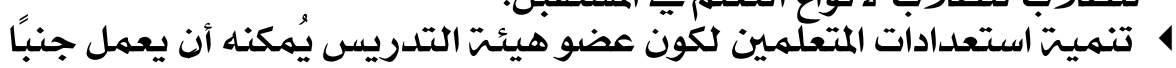

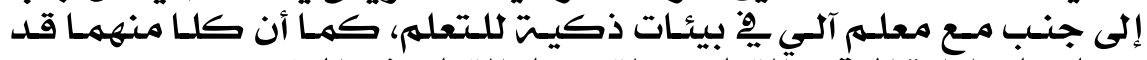

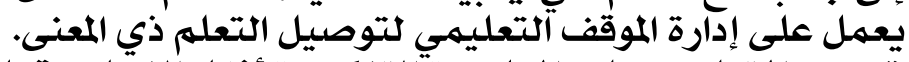

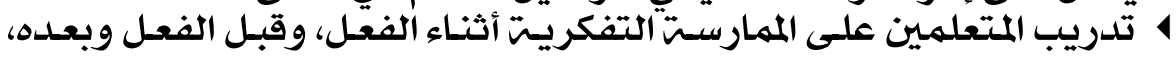

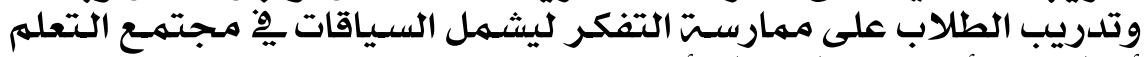

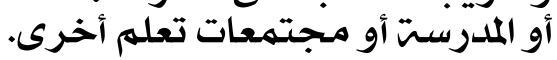

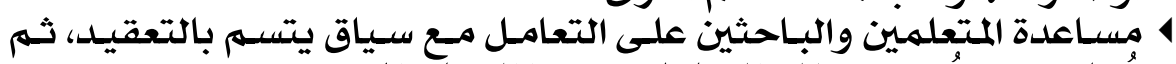

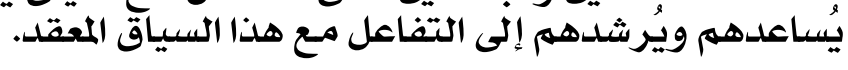

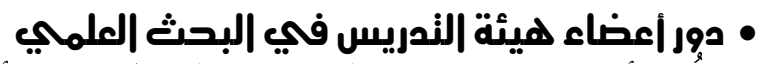

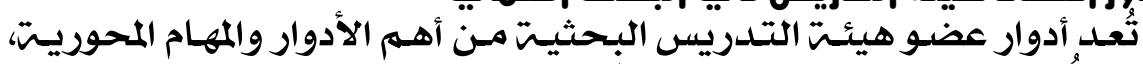

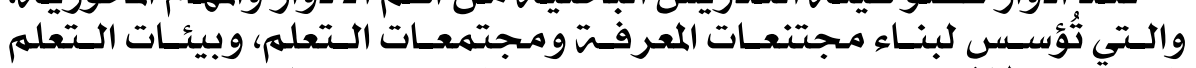

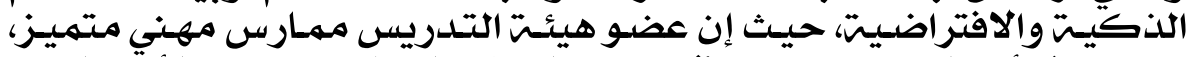

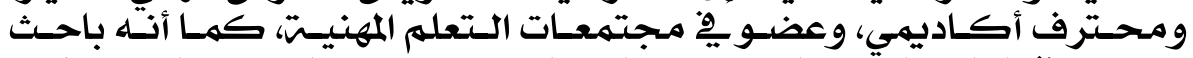

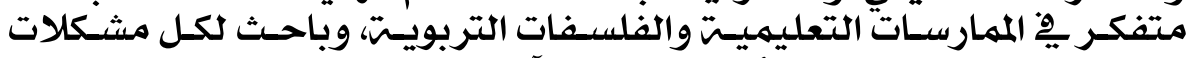

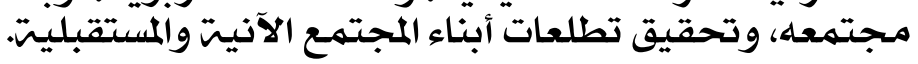

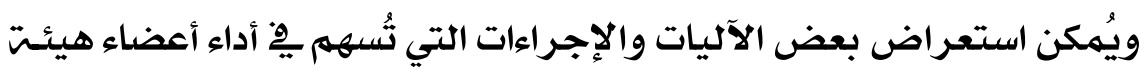

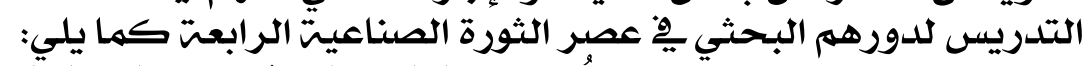

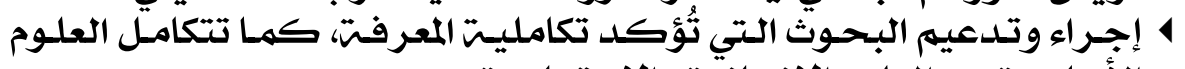

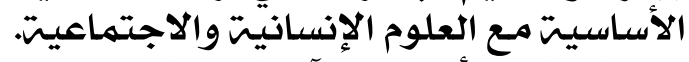

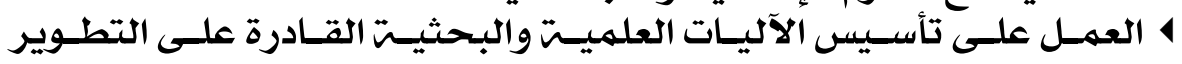

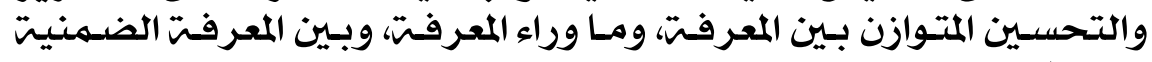

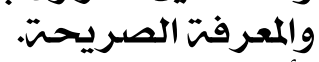

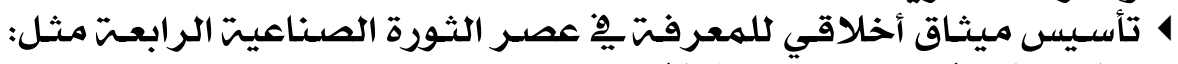

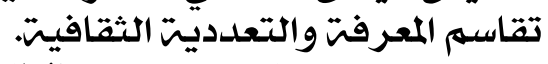

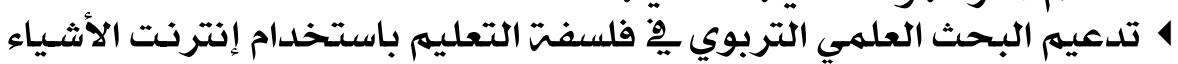

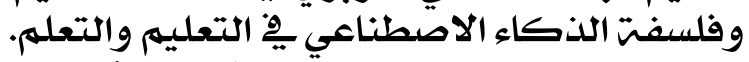

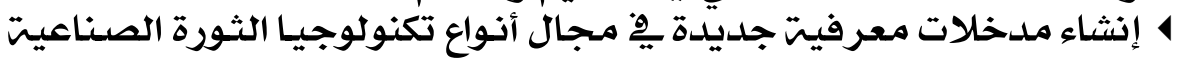
الرابعتش.

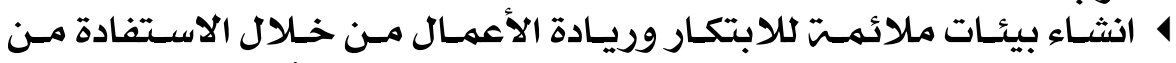

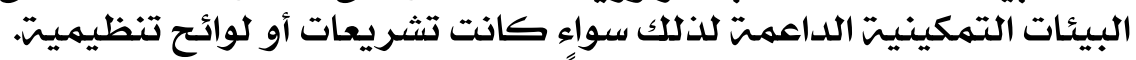

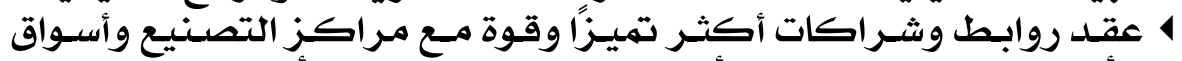

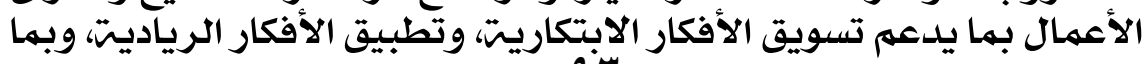
و 


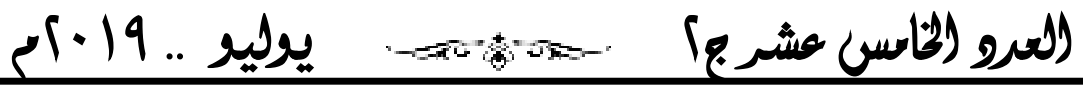

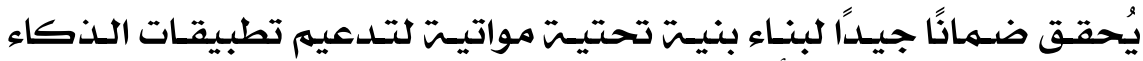

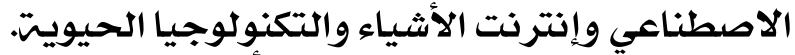

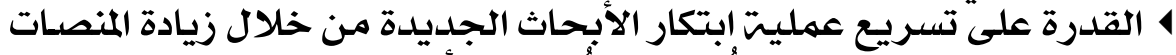

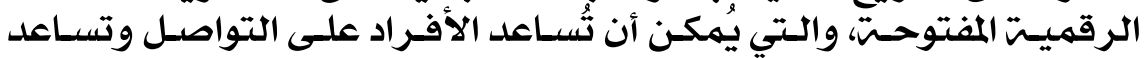

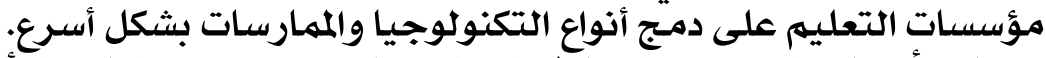

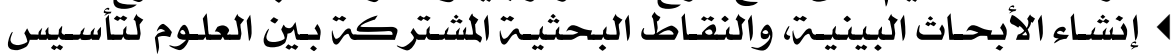

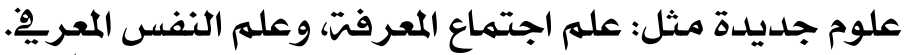

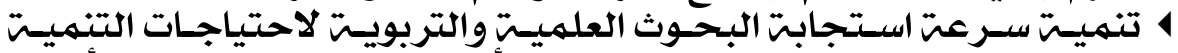

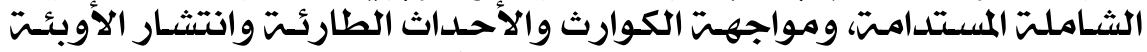

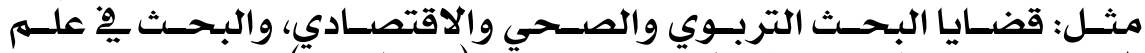

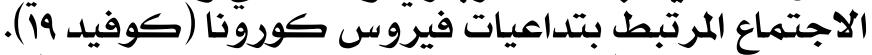

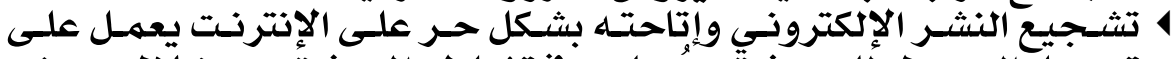

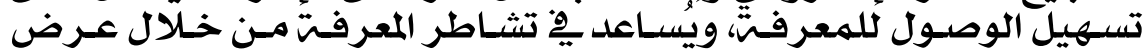

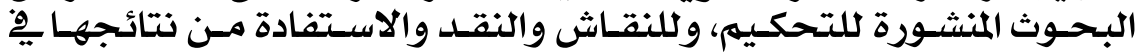
إنشاء معارف جلديدة.

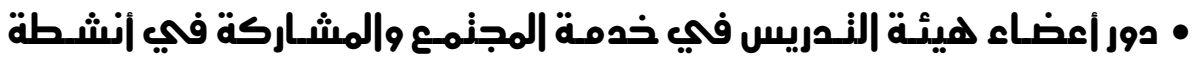

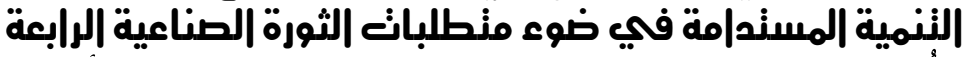

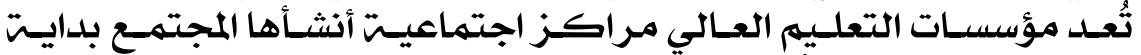

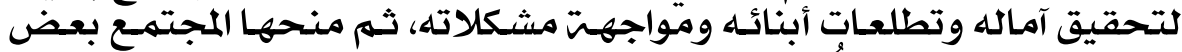

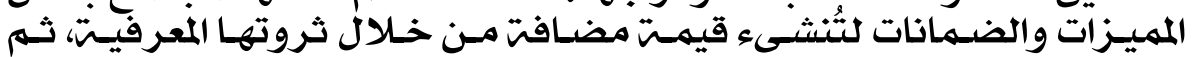

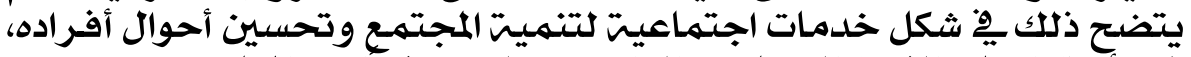

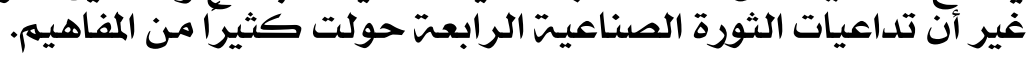

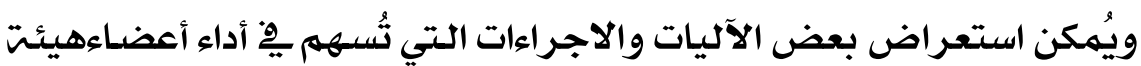

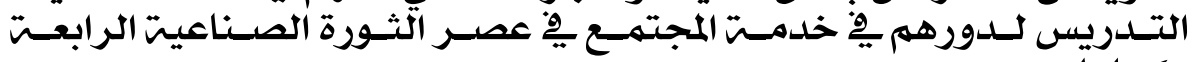
كبها بلمي:

4 التوسـع هِّ إجراء الدراسـات التي تُعالج قضايا المجتهـع وتحدياته.

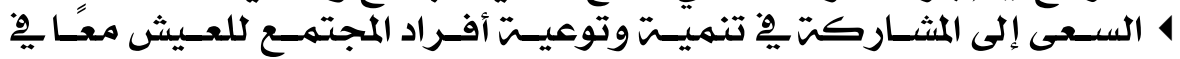

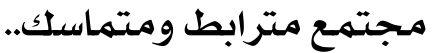

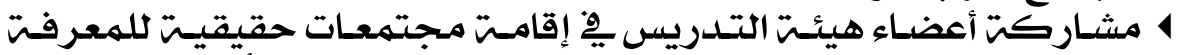

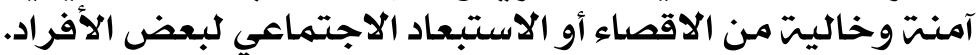

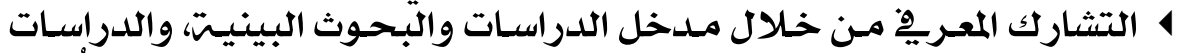

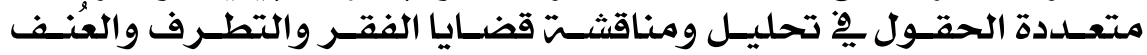
وتغييرات المناخ.

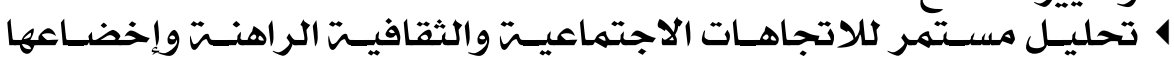

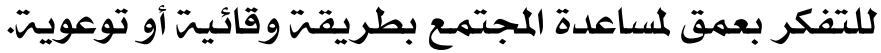

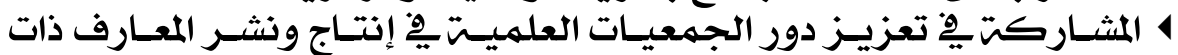

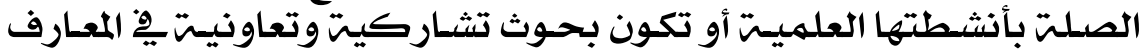

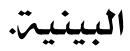




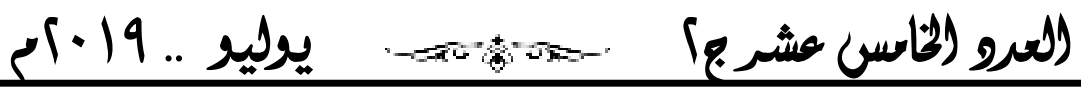

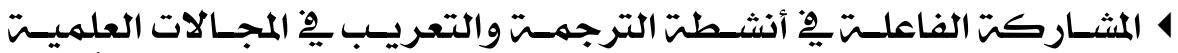

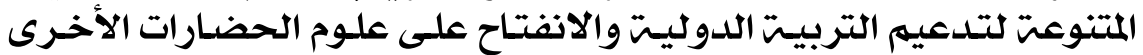
وثقافاتهم.

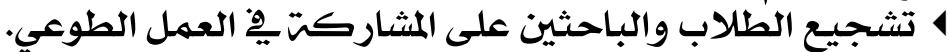

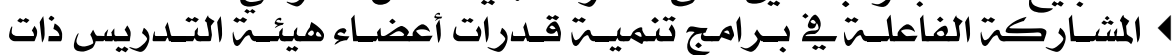

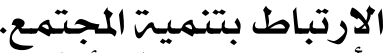

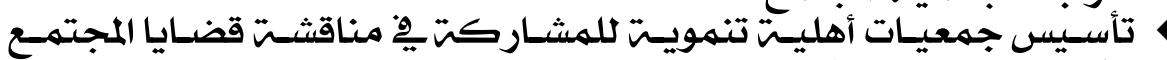

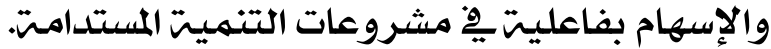

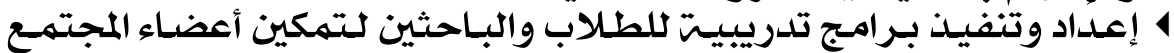

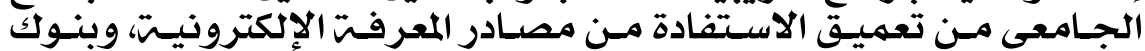

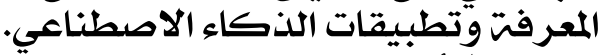

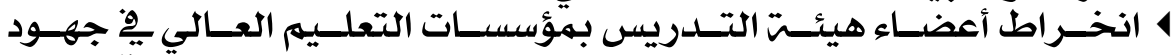

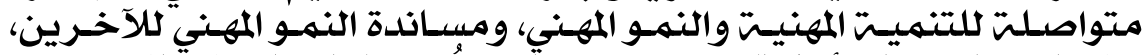

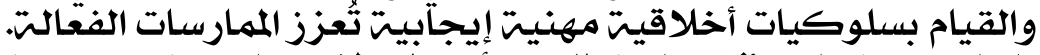

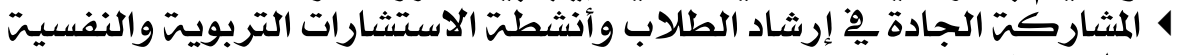
والاجتمعاعيت.

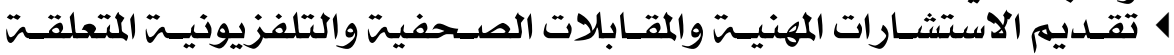

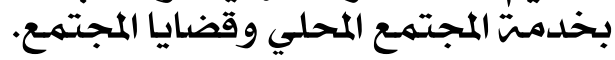

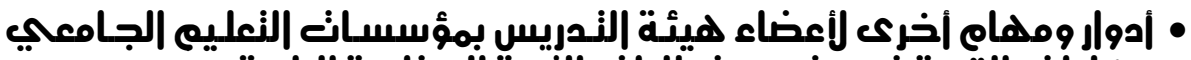

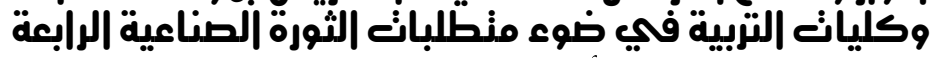

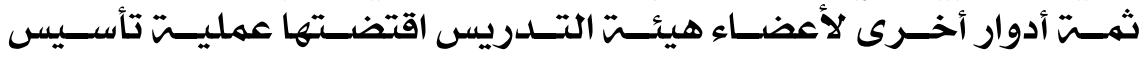

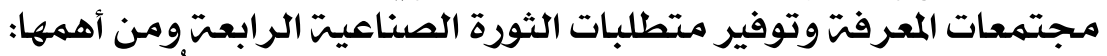

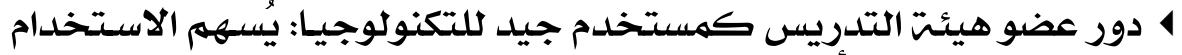

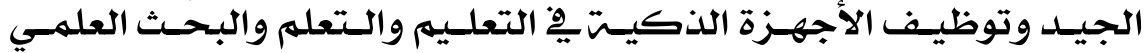

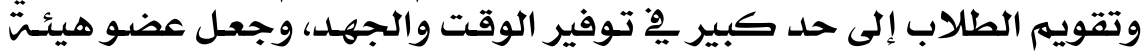

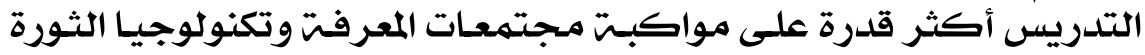
الصناعيت الرابعت.

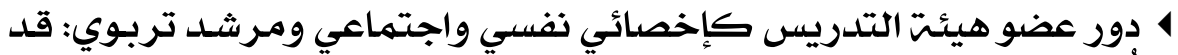

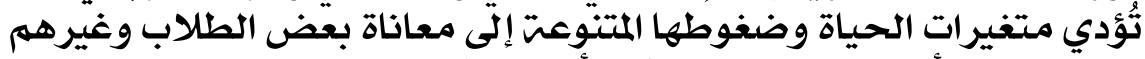

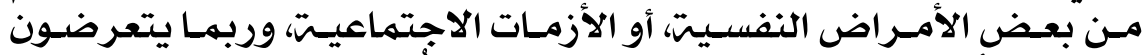

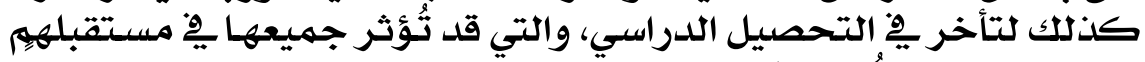

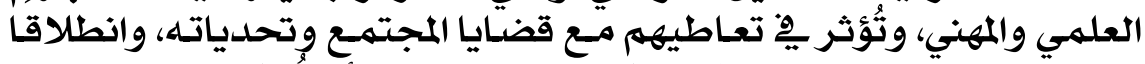

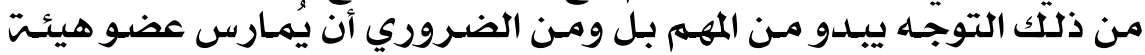

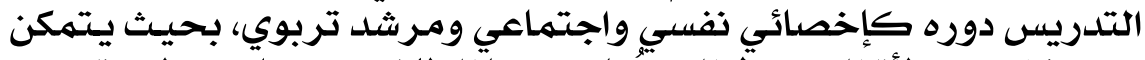

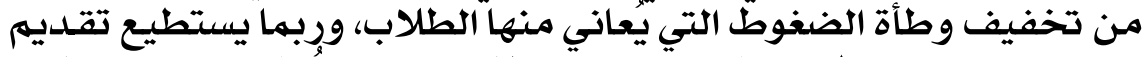

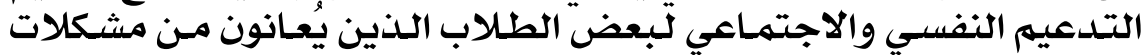

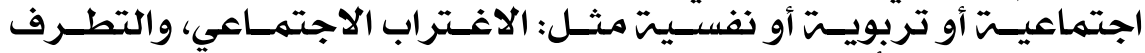

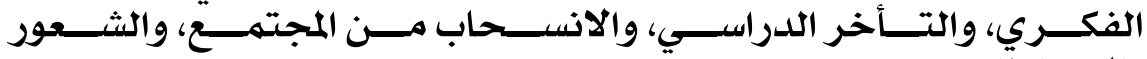

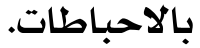




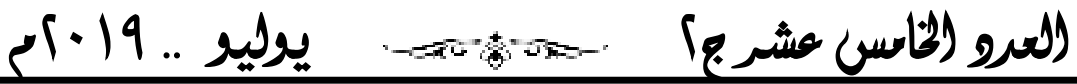

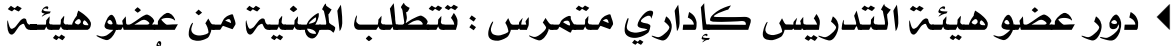

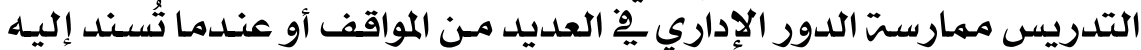

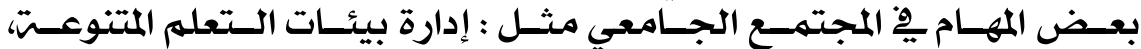

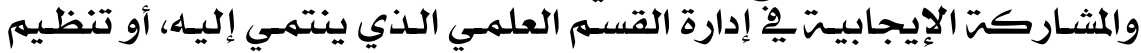

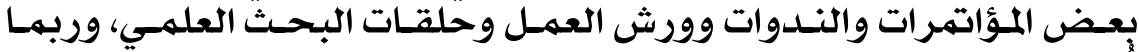

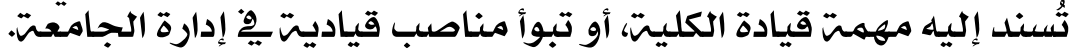

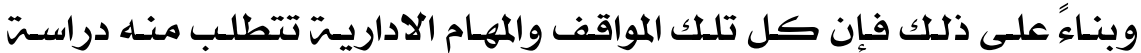

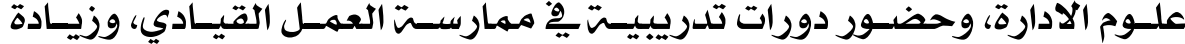

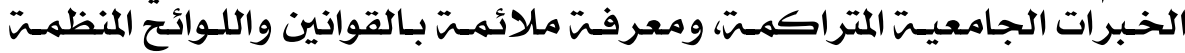
للعمل بالجأمعتة.

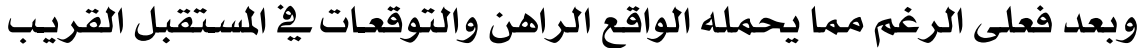

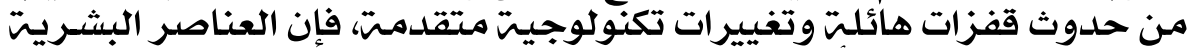

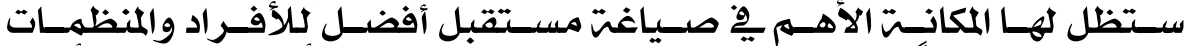

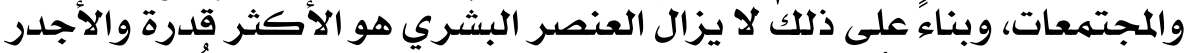

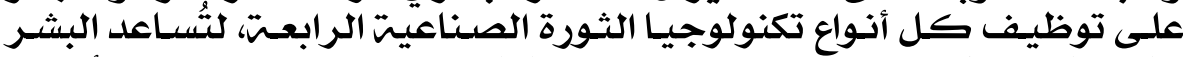

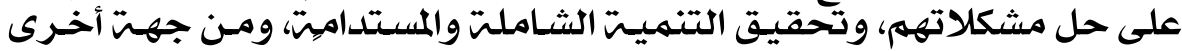

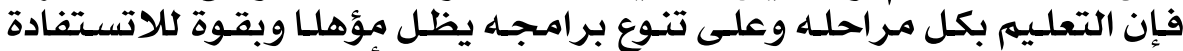

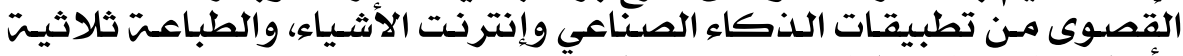

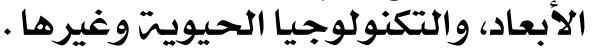

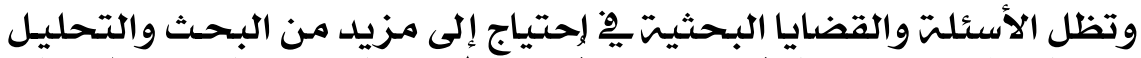

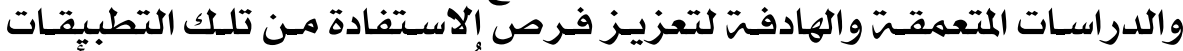

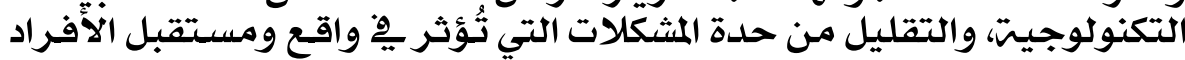

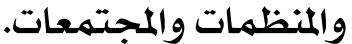

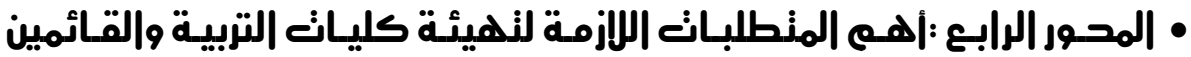

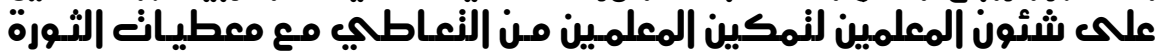
|لصناعية الرابعة الهونة

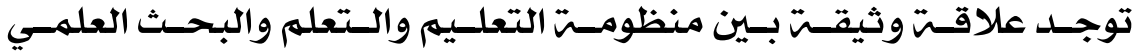

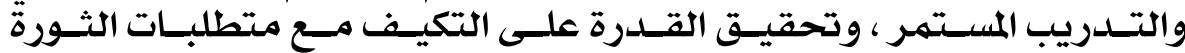

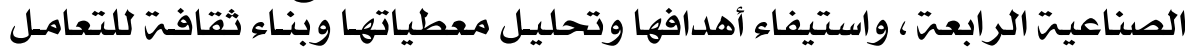

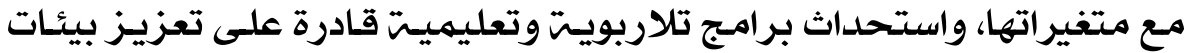

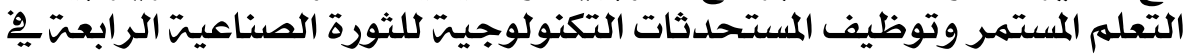

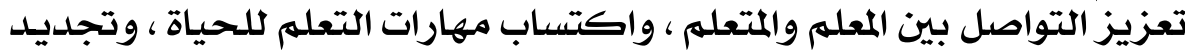

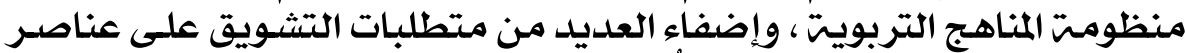

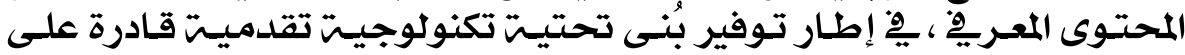

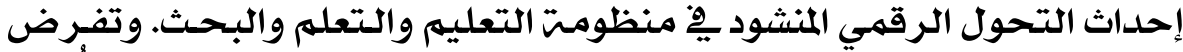

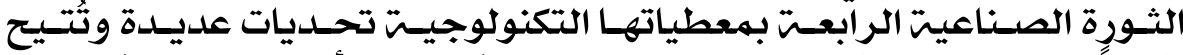

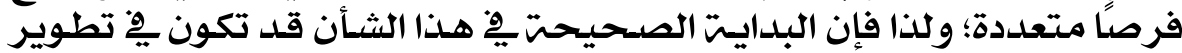




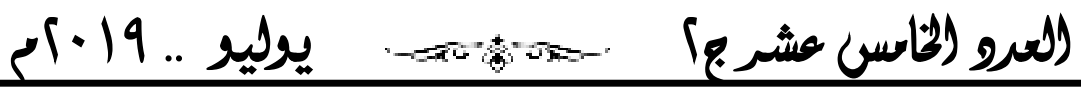

كليـات التربيـت وتحسـين قدرتها على الاسـتجابت لمتطلبـات الثورة الصسناعيت

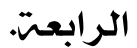

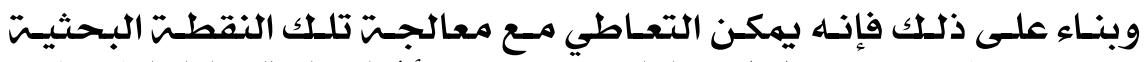

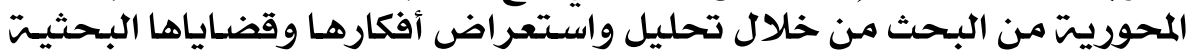

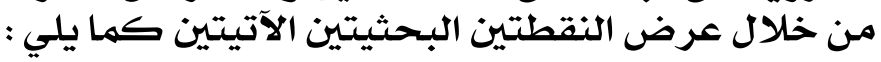
I - منطابانه نمكين الهملهين ذانه ارنباط بسياسانه فمالة لنمكين الهملهين

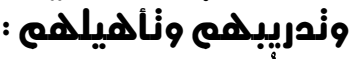

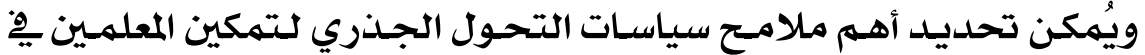

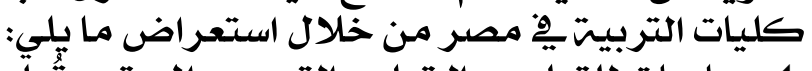

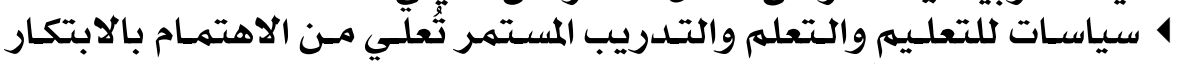

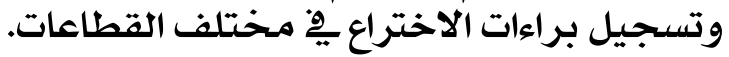

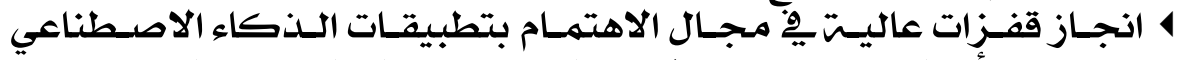

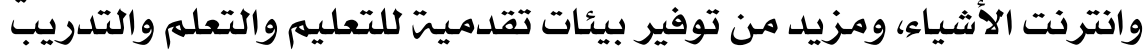

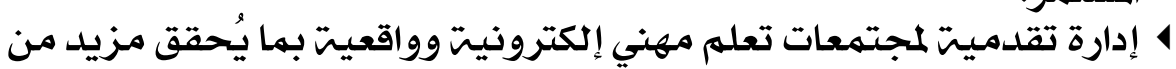

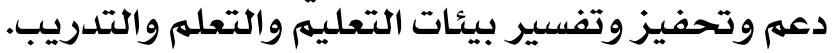

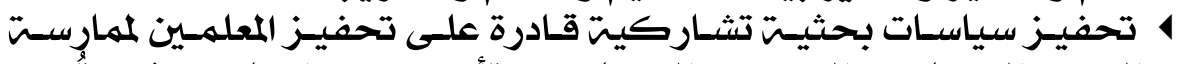

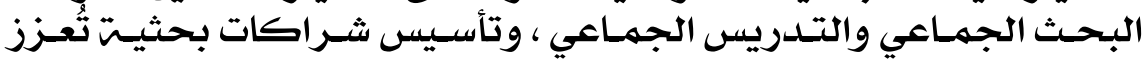

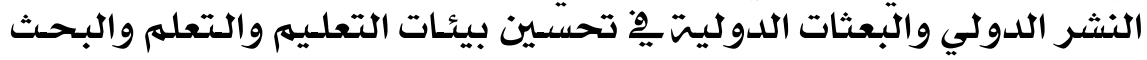

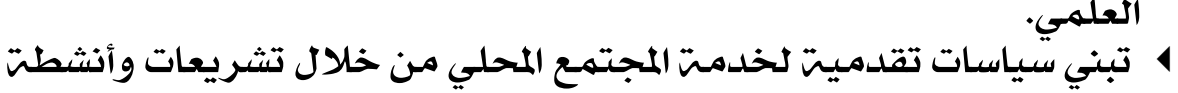

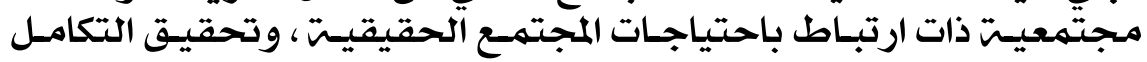

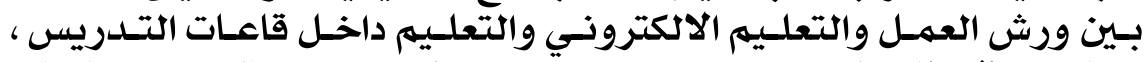

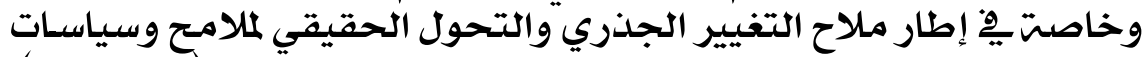

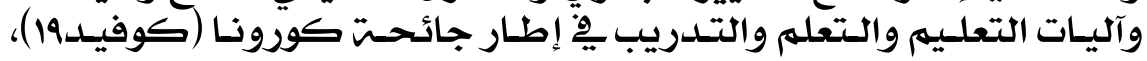

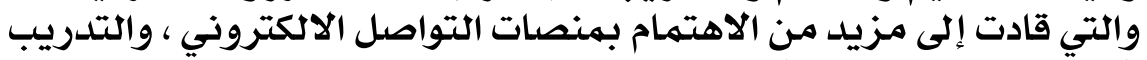

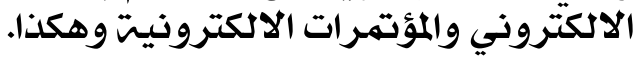

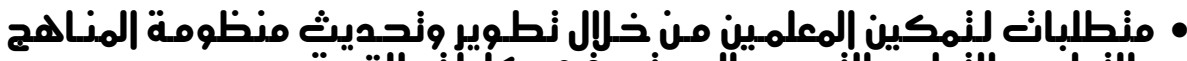

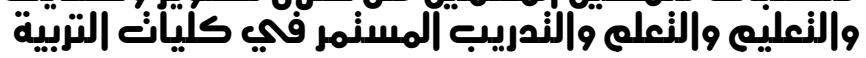

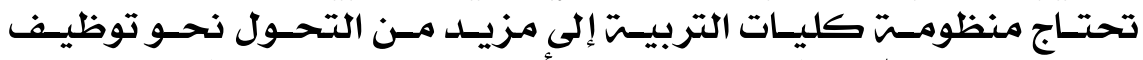

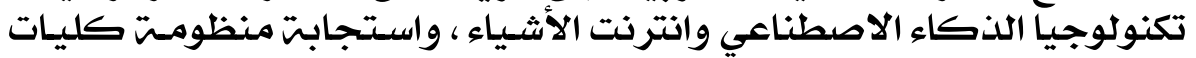

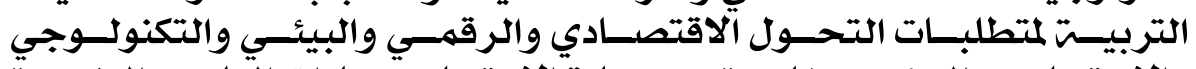

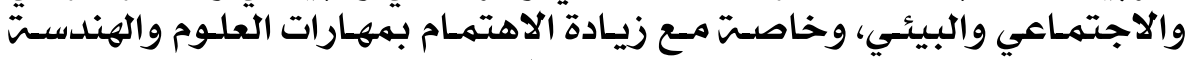

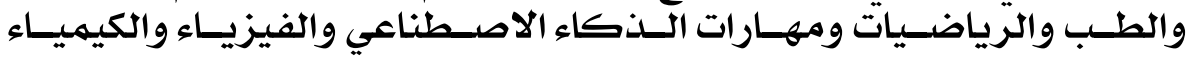

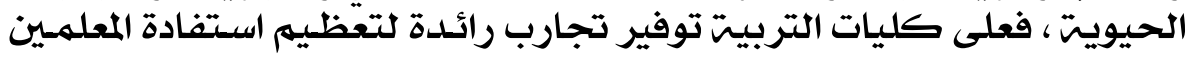

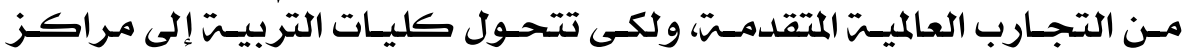

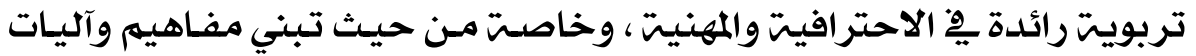
$9 \mathrm{~V}$ 
(العرو (الخامس عشرج) .

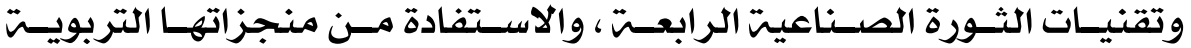

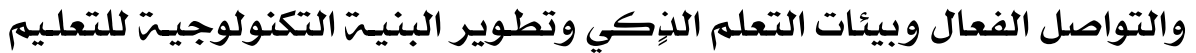

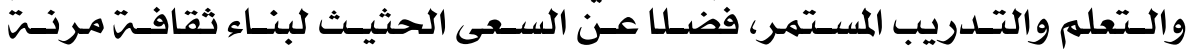

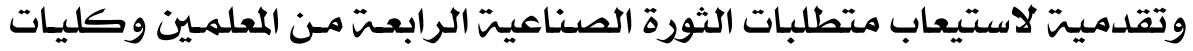

إعداد المحلهم.

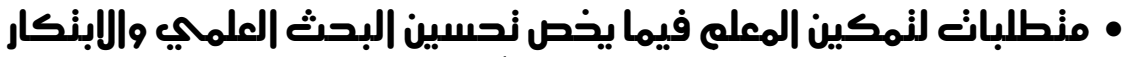

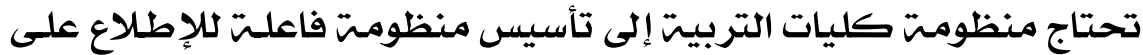

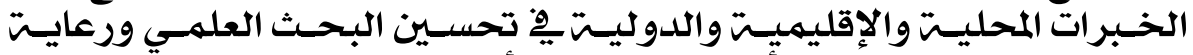

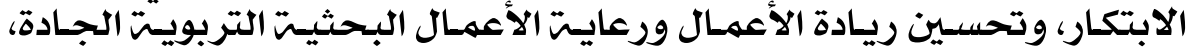

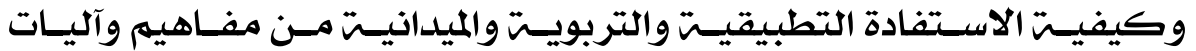

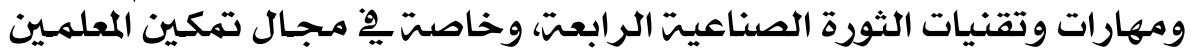

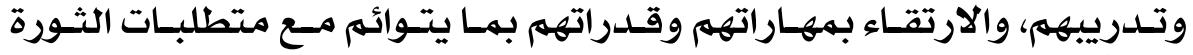

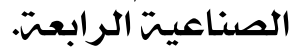

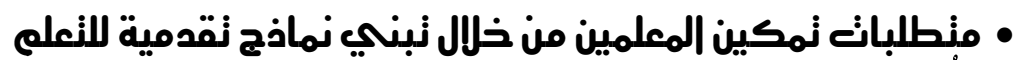

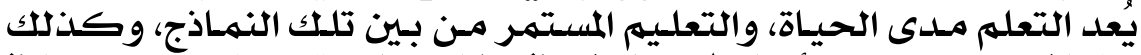

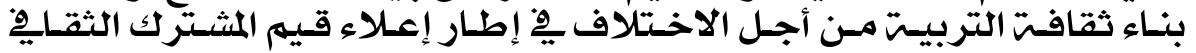

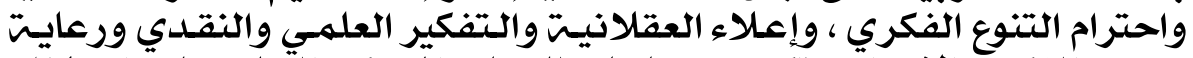

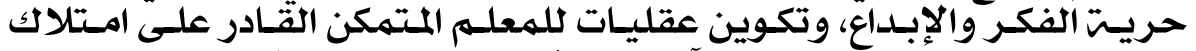

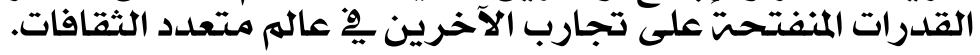

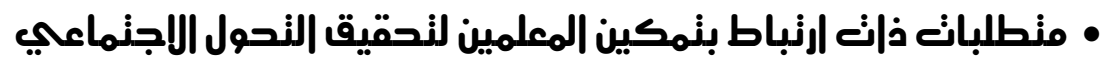

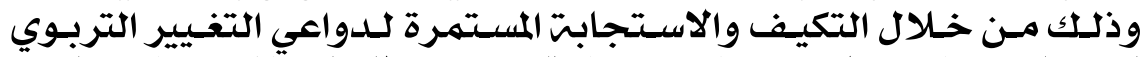

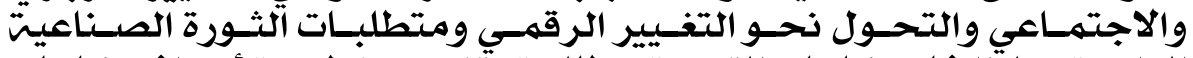

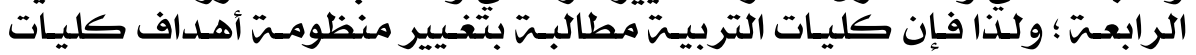

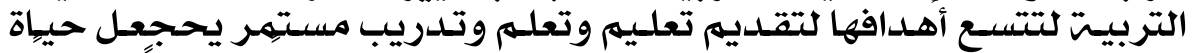

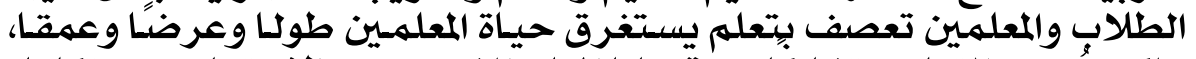

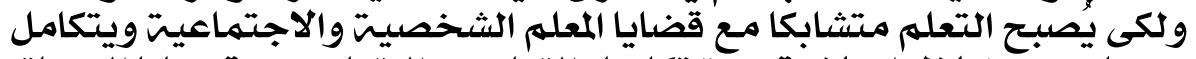

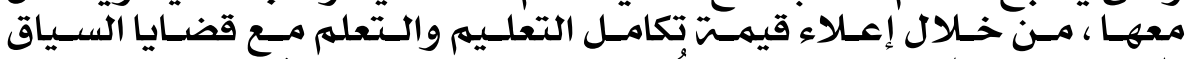

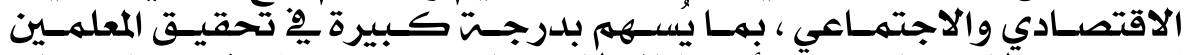

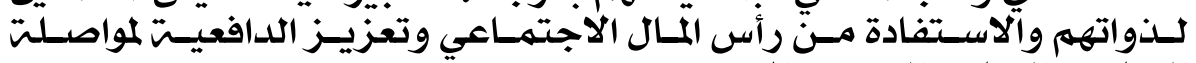

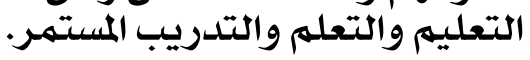

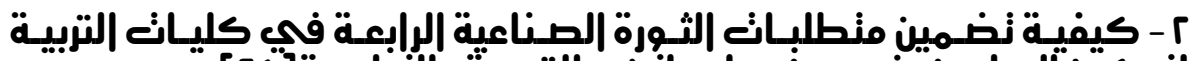

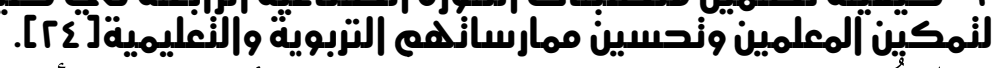

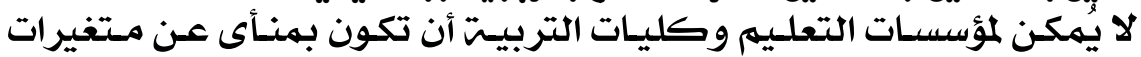

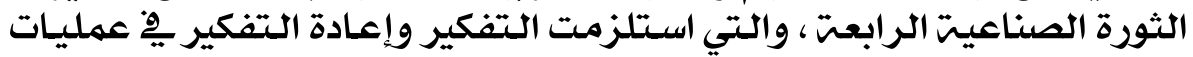

24- Bernard Marr: 8 Things Every School Must Do To Prepare for the 4th Industerial Revolution Retreaved From https://www.forbes .com/sites/ bernardmarr/2019/05/22/8-things-every-school-must-do-to-prepare-for-the-4thindustrial-revolution/\#1fccd8ab670c

$9 \wedge$ 


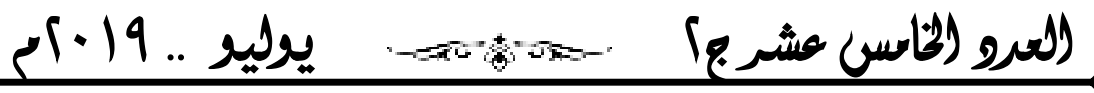

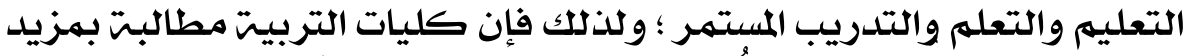

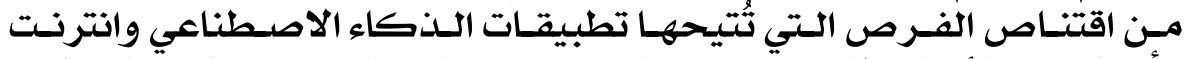

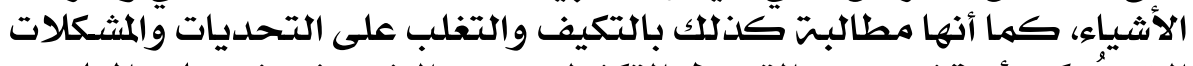

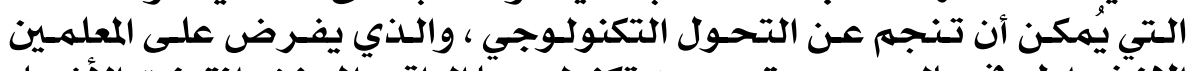

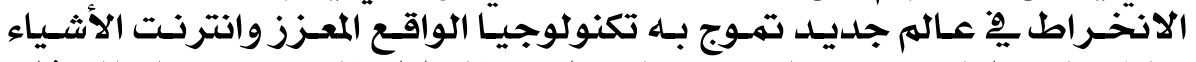

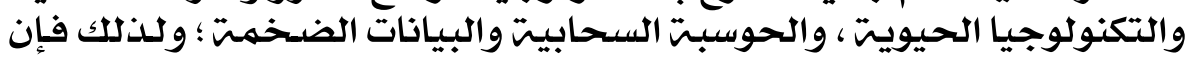

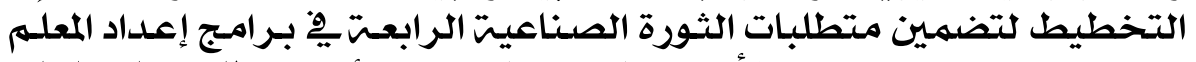

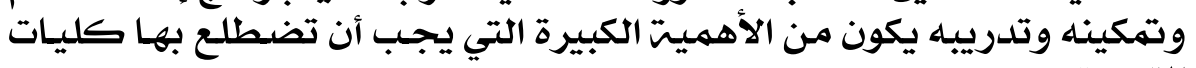
التربينت التهينه

ومن أهم آليات تضمـين متطلبات الثورة الصناعيت بكليات التربيت ما يلي:

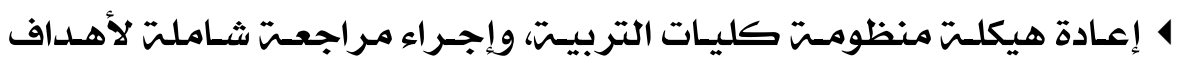

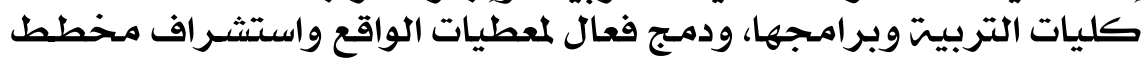

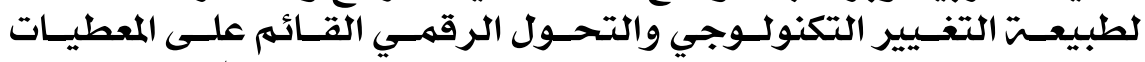

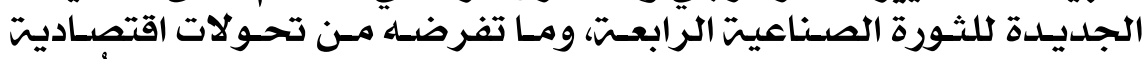

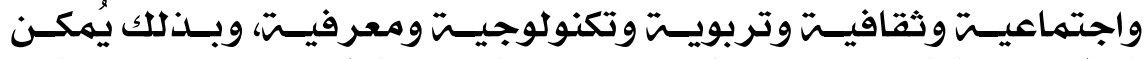

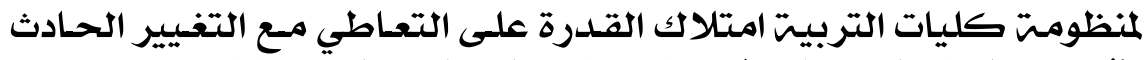

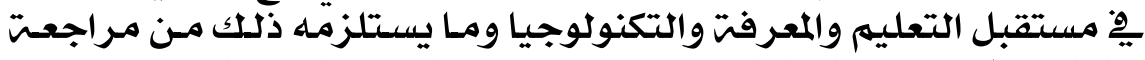

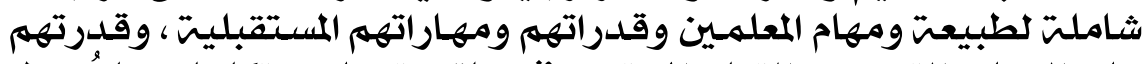

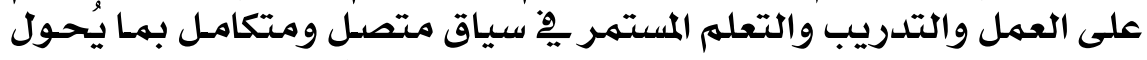

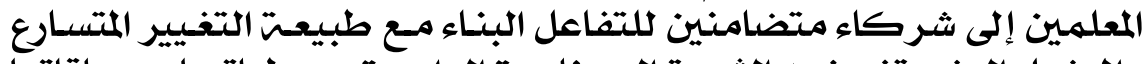

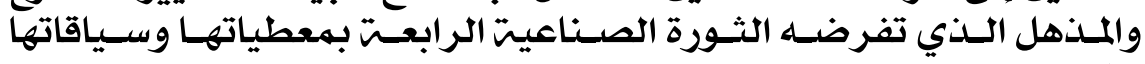

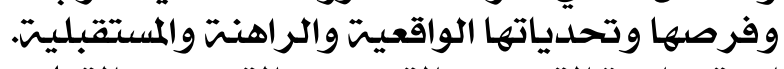

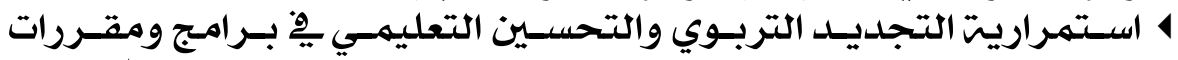

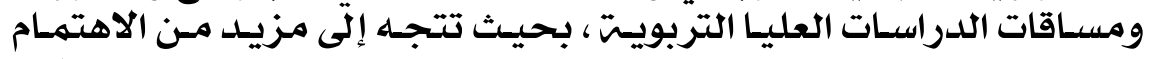

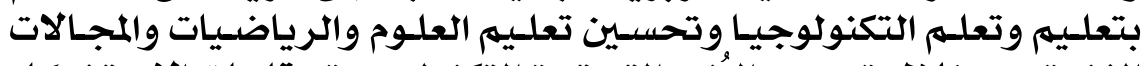

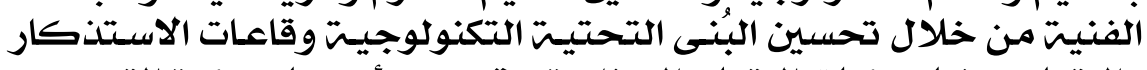

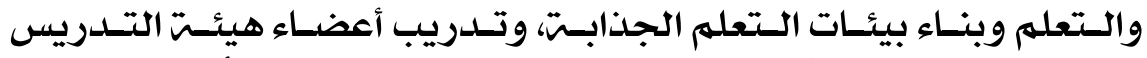

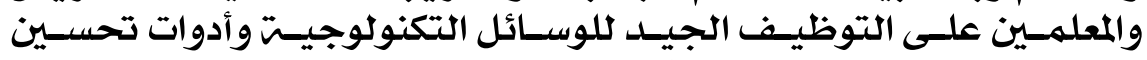

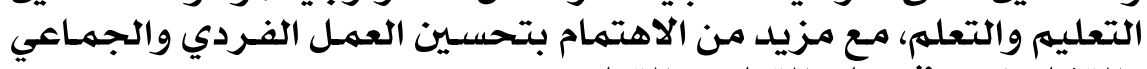

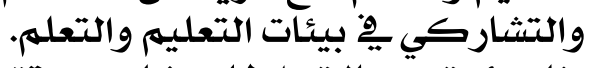

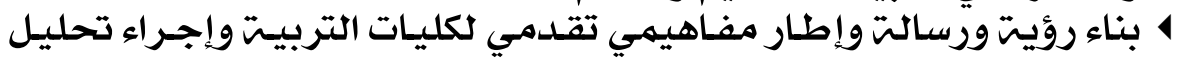

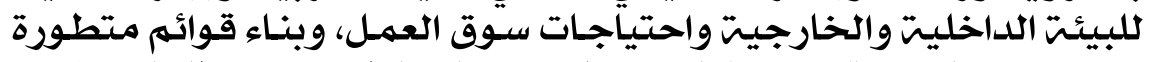

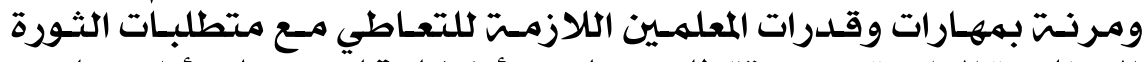

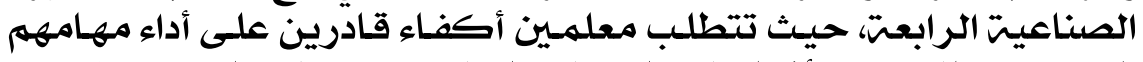

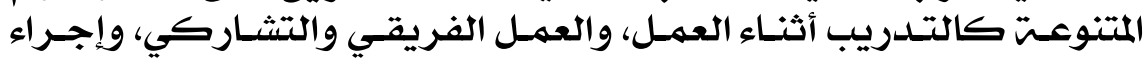

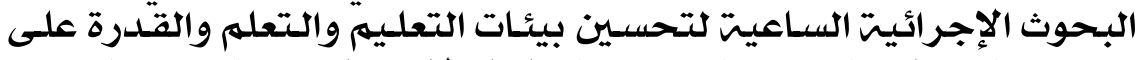

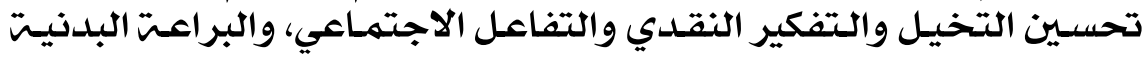

99 


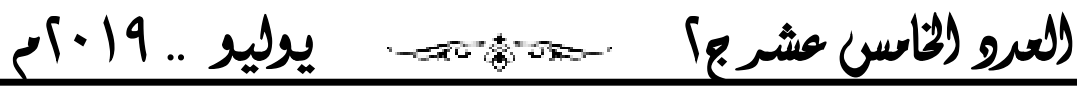

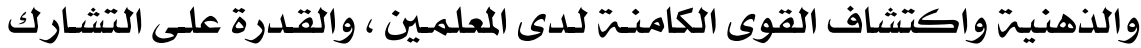

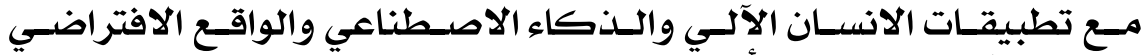

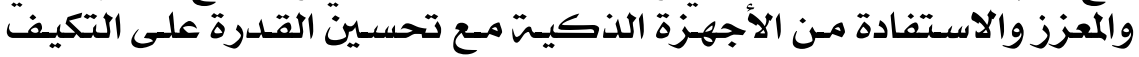

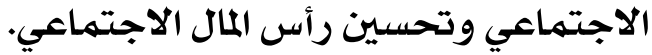

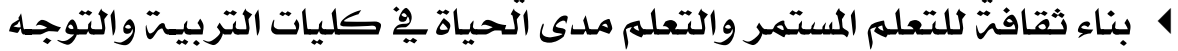

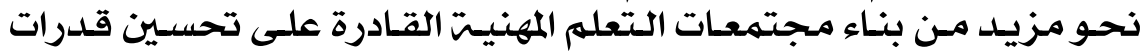

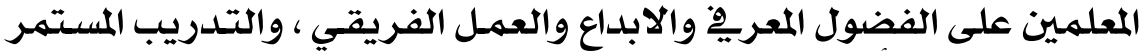

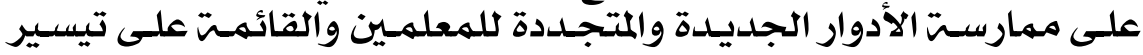

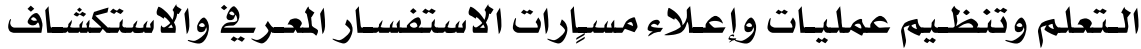

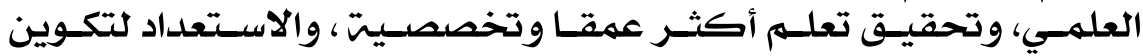

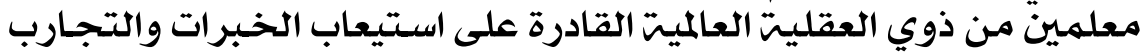

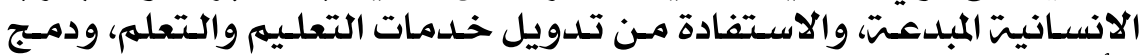

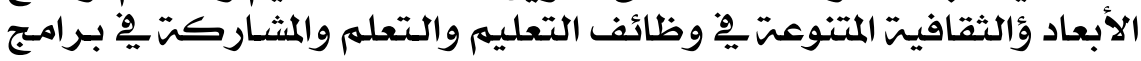

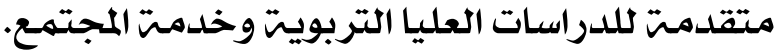

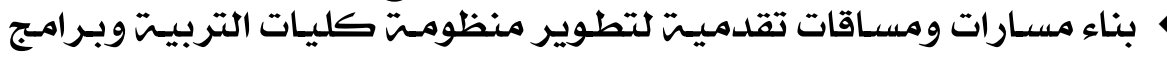

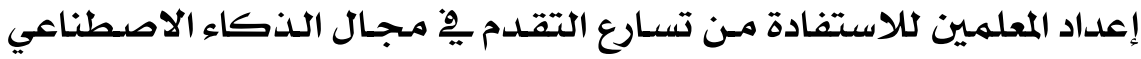

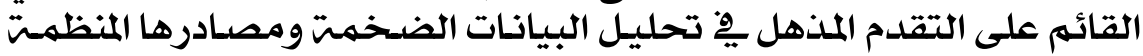

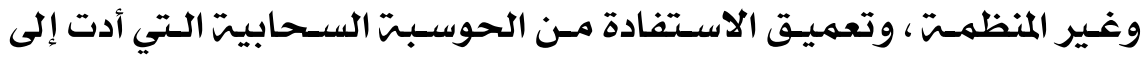

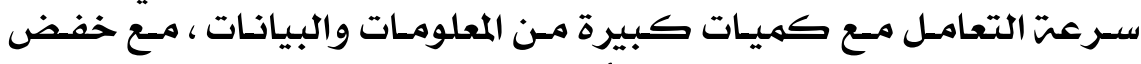

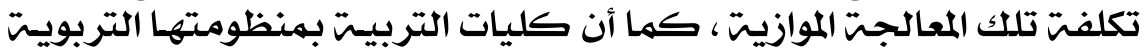

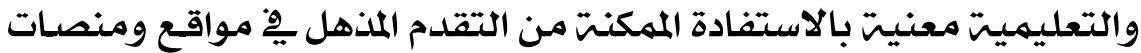

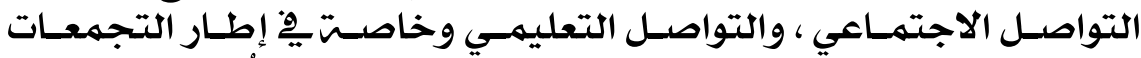

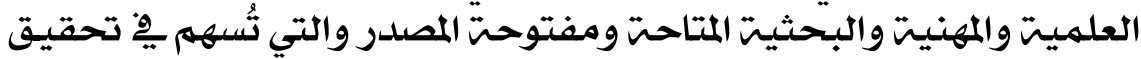

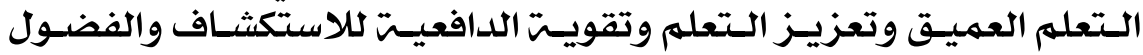

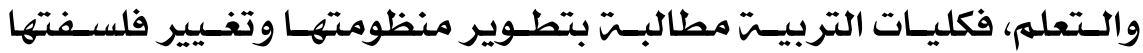

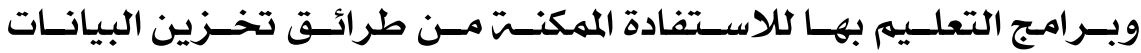

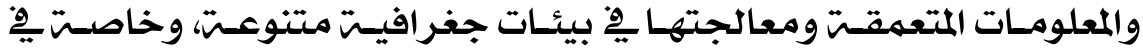

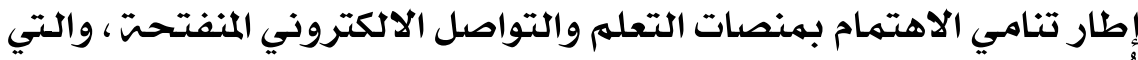

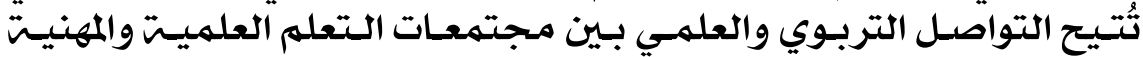

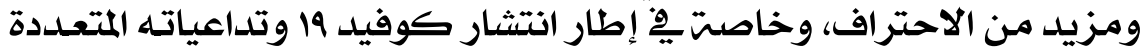

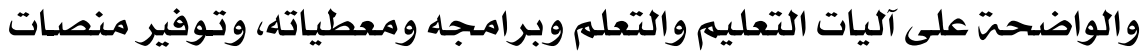

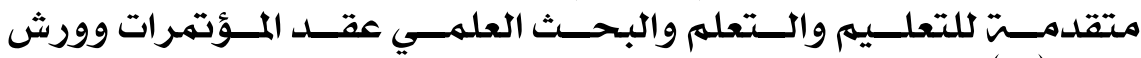

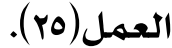

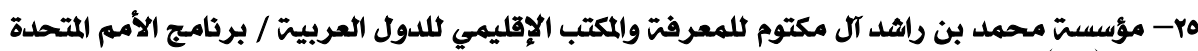

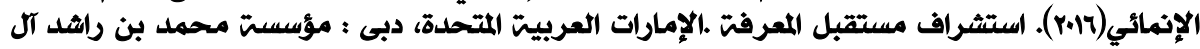


(العرو (الخامس عشرج) .

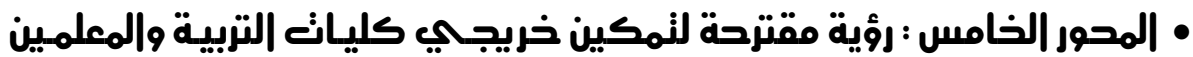

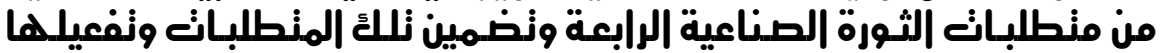
في العملية النعليمية نطبيقا وممارسة

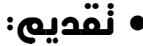

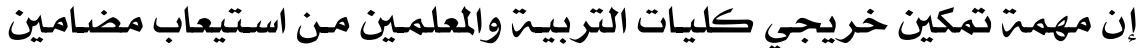

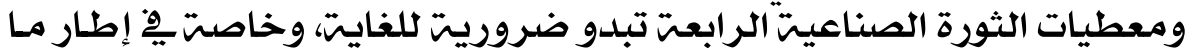

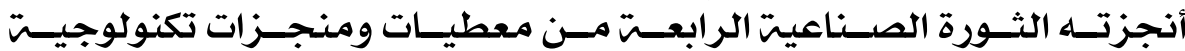

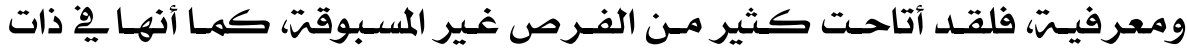

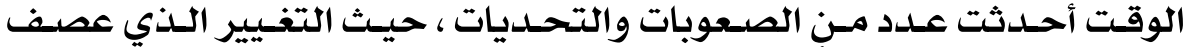

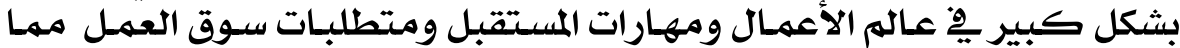

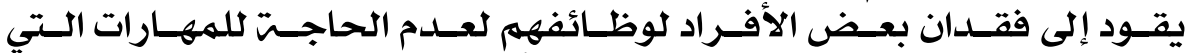
يهتلكونها، فالتكنولوجيا الحديثت كفيلت بأدائها.

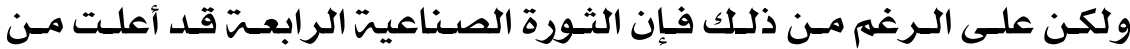

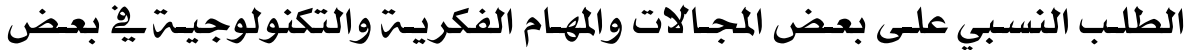

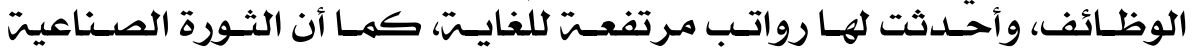

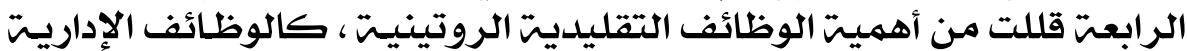

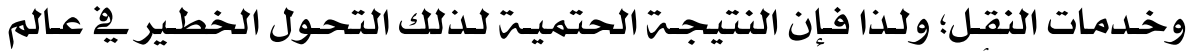

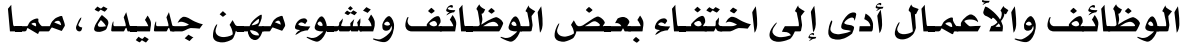

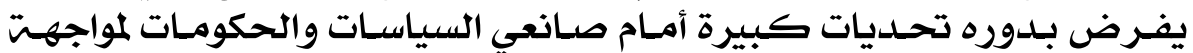

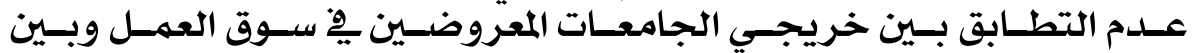

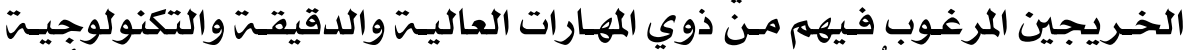

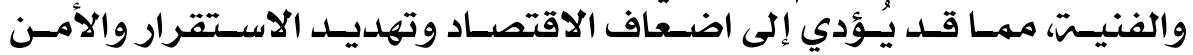
الاجتمعاعي.

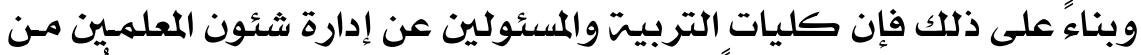

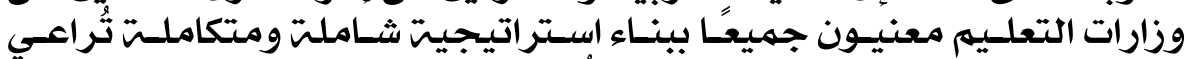

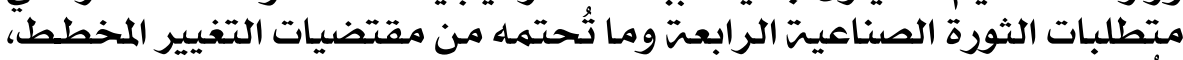

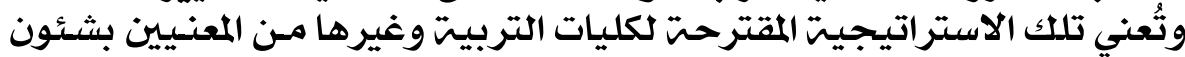

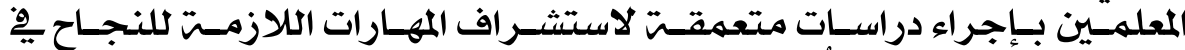

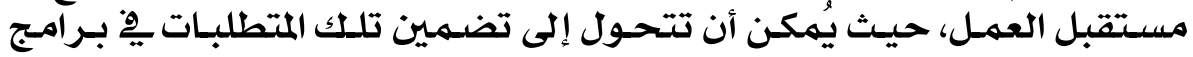

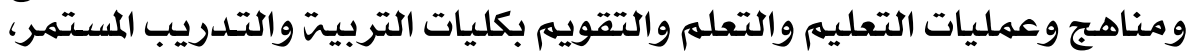

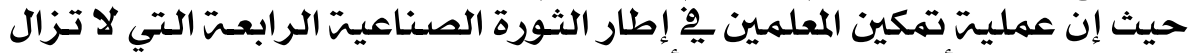

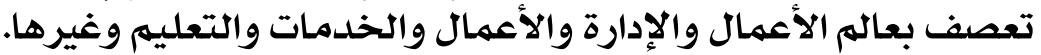

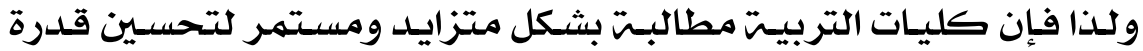

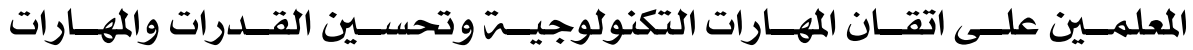

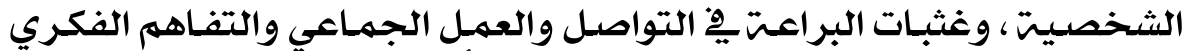

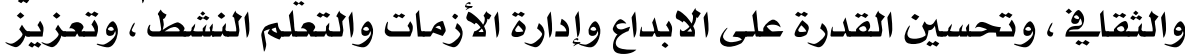




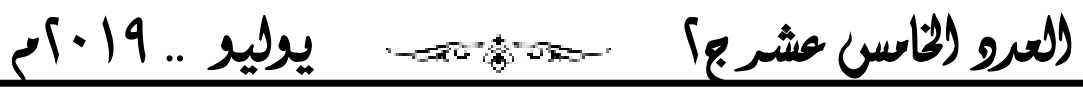

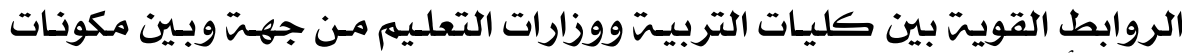

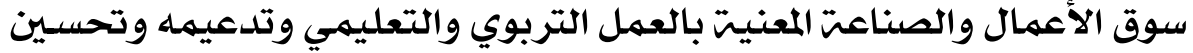

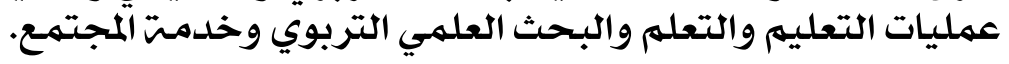

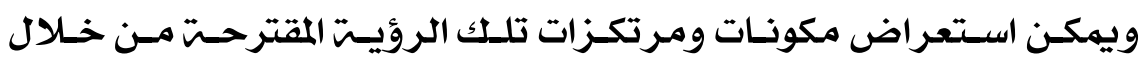

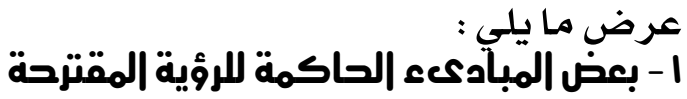

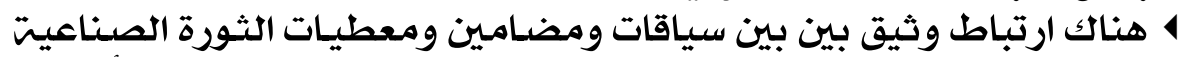

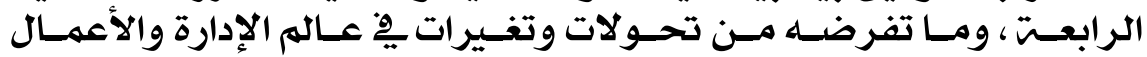

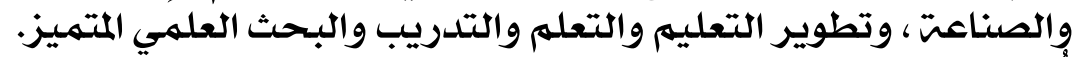

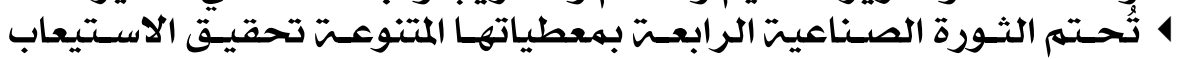

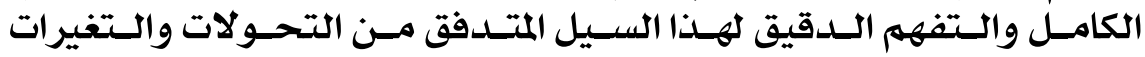

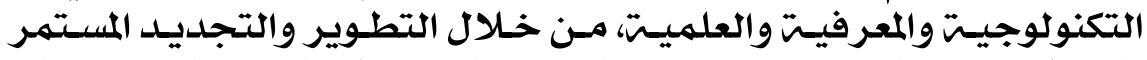

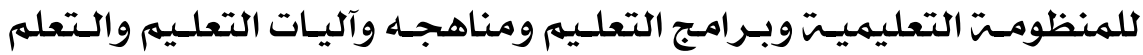

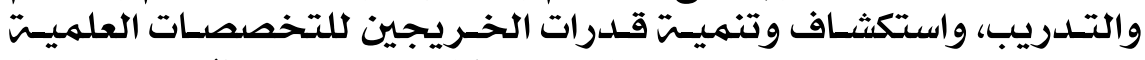

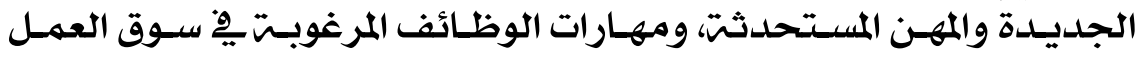

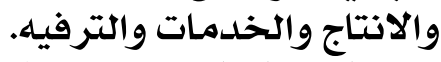

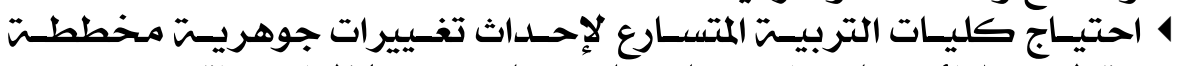

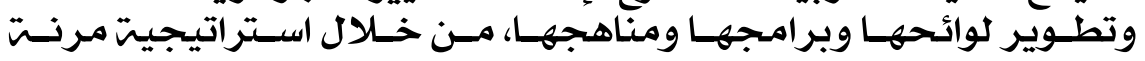

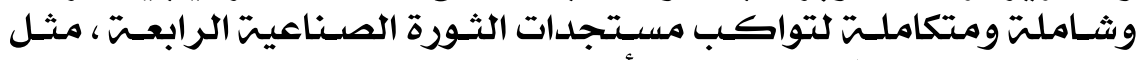

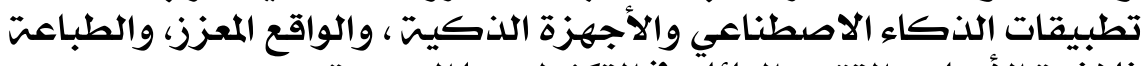

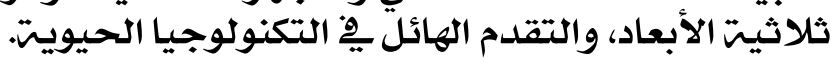

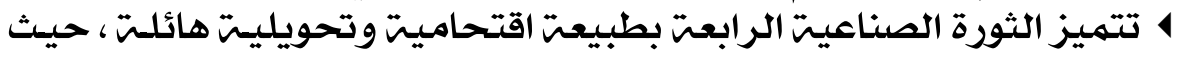

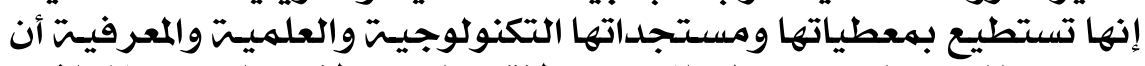

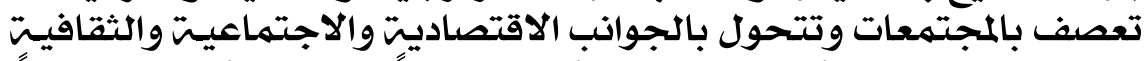

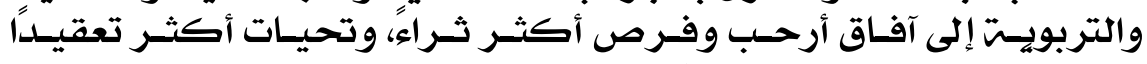

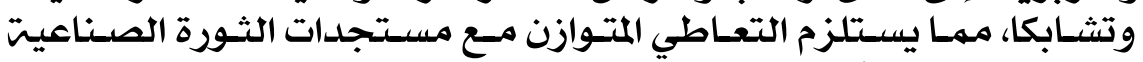

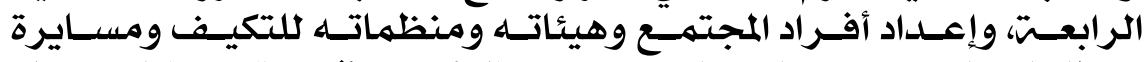

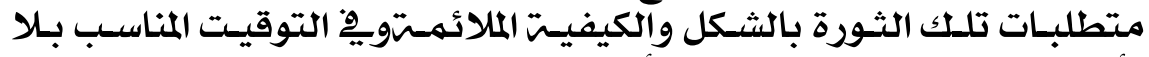

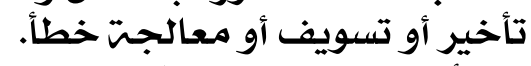

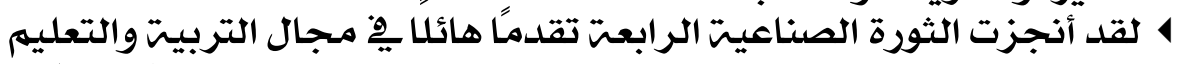

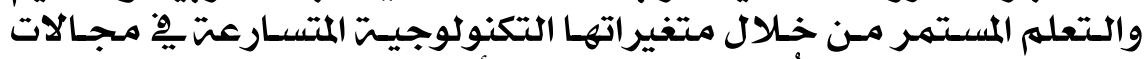

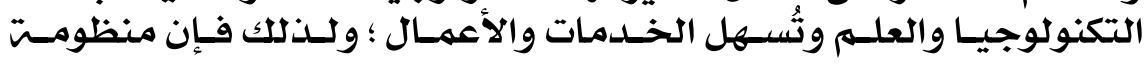

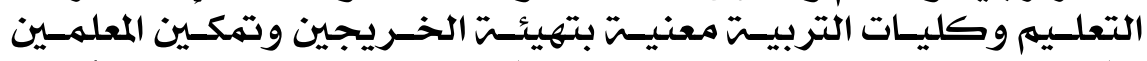

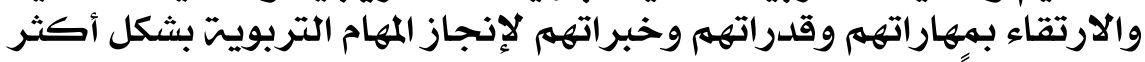
جودة وتميزًا.

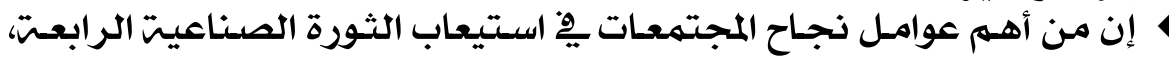

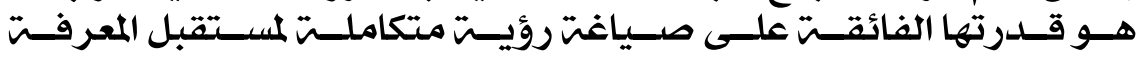




\section{(لعرو (لخاس) عشرج) .}

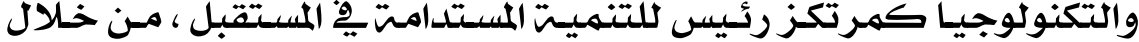

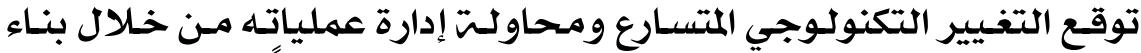

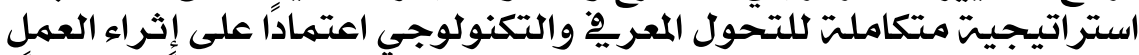

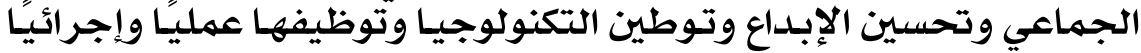

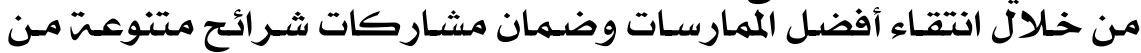

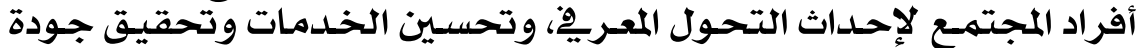

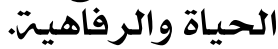

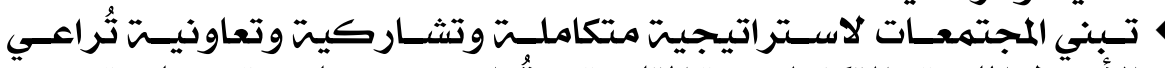

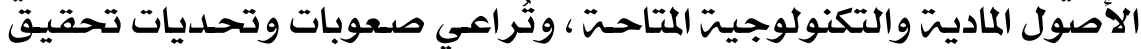

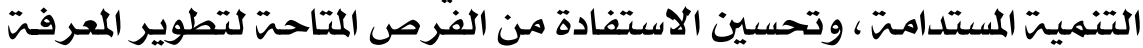

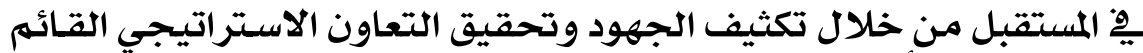

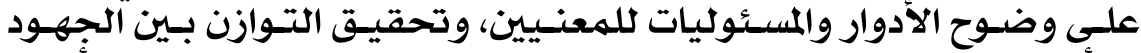

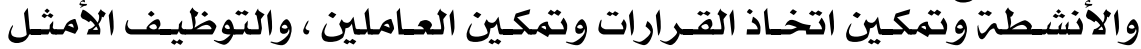

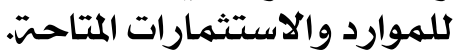

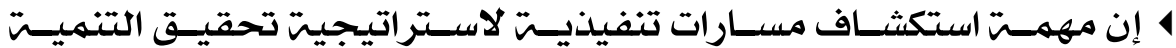

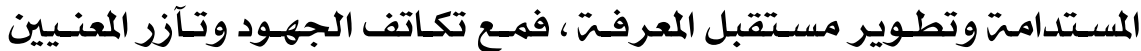

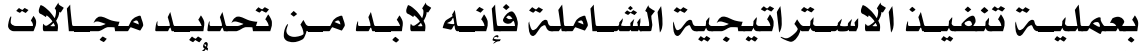

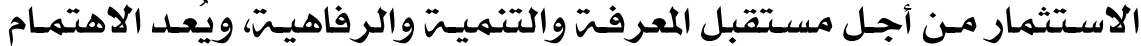

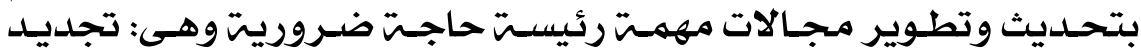

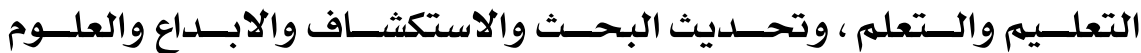

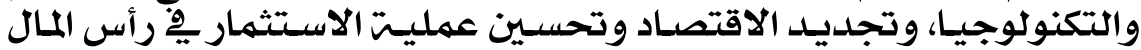

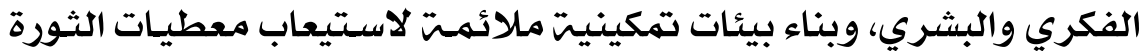

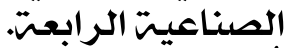

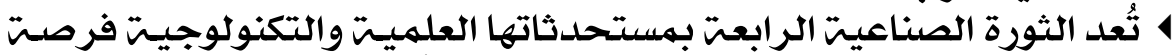

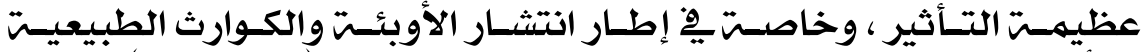

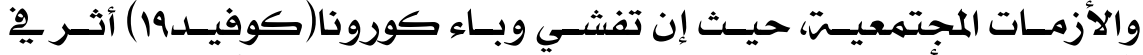

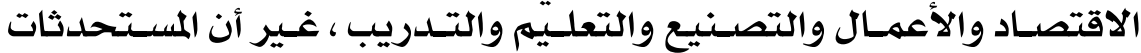

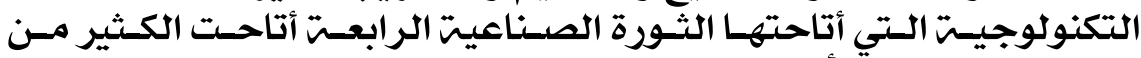

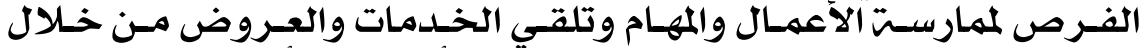

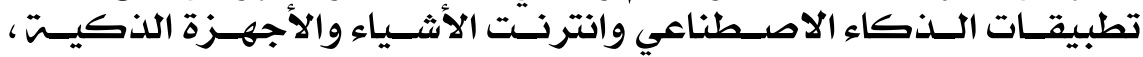

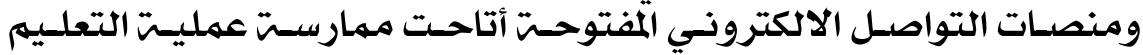

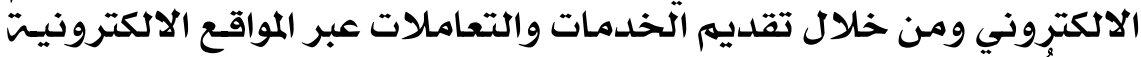
، مما يقلل من انتشار الوباء إلى حلد ما.

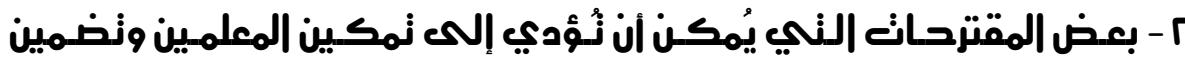

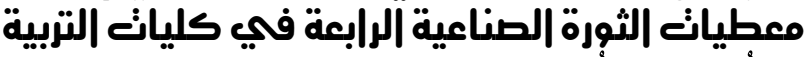

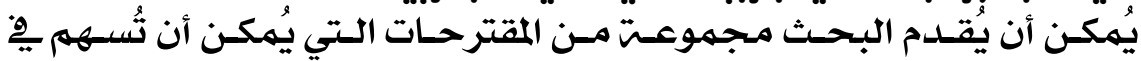

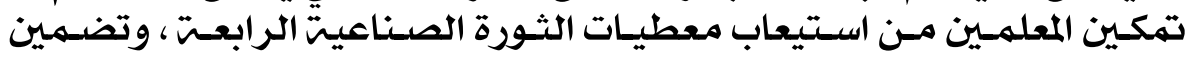


(العرو (الخاس) عشرج) .

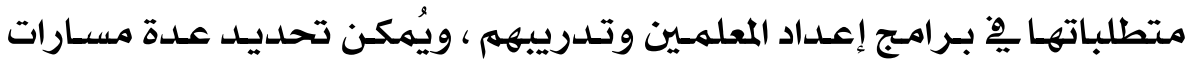

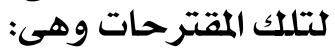

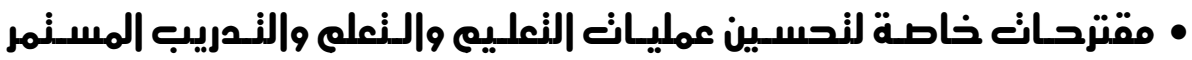

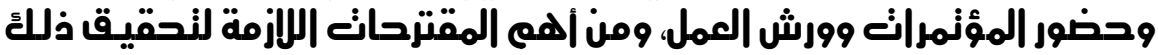

ما يلي:

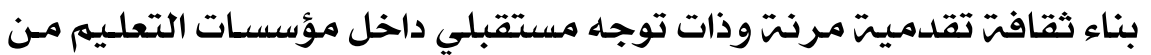

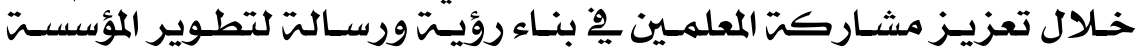

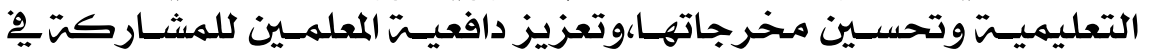

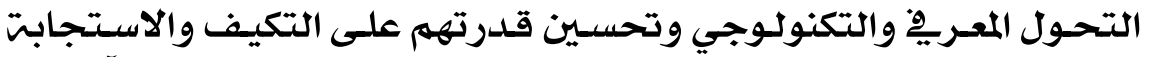

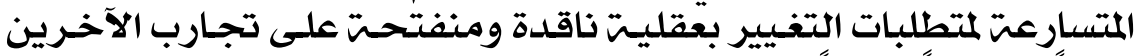

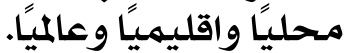

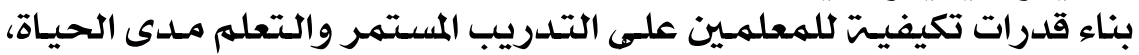

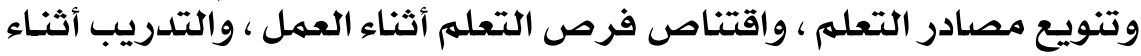

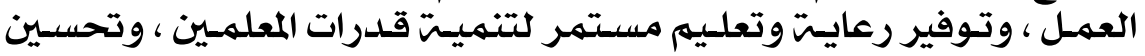

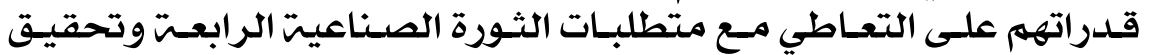
متطلبات مدرست المستقبل.

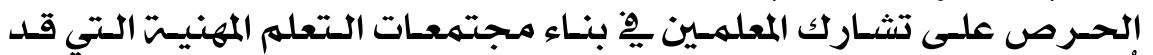

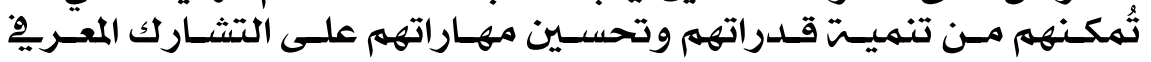

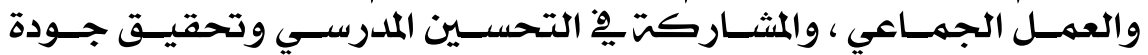
المؤسسات التعليميتي.

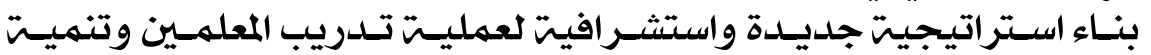

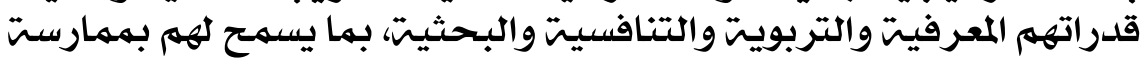

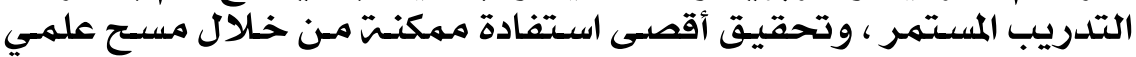

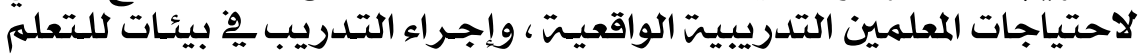

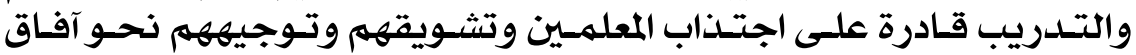

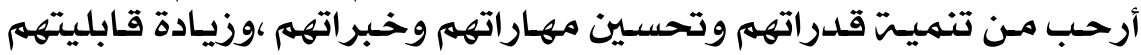

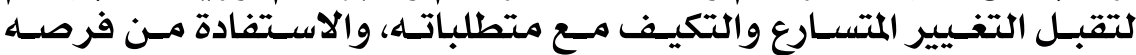

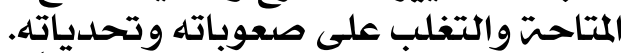

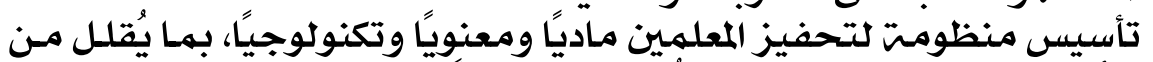

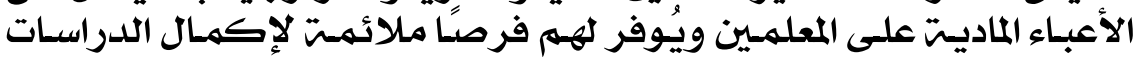

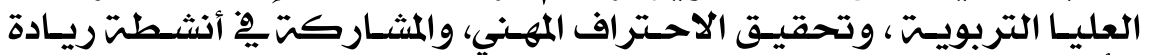

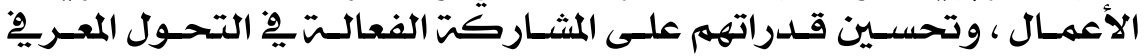
والتكنولوجي.

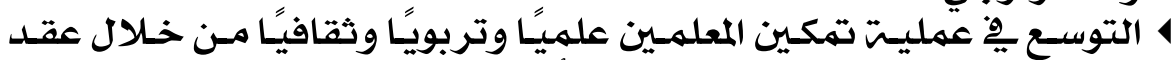

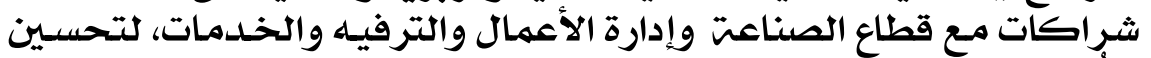

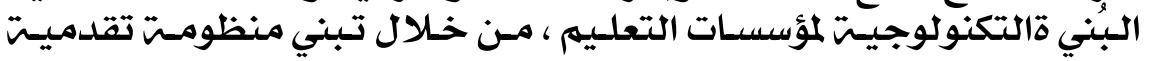

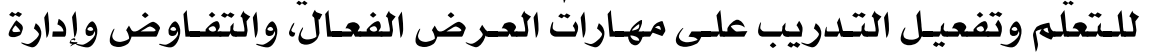

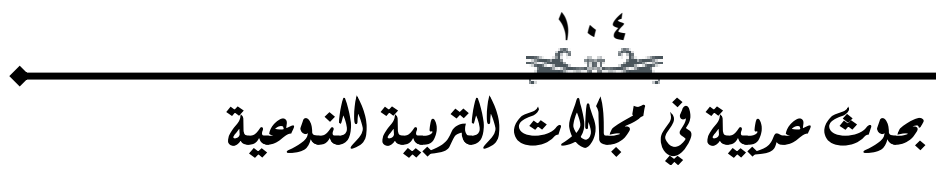




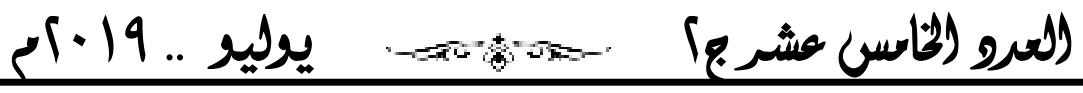

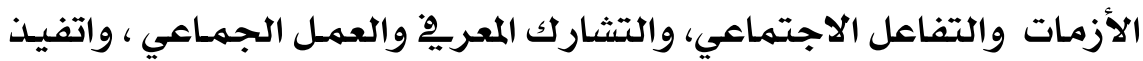
مشروعات رياديت ناجحت والتهاعت وفيالت.

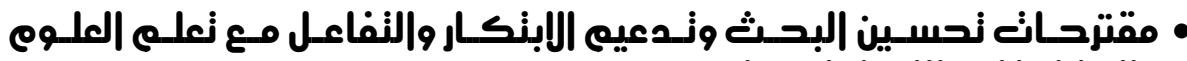

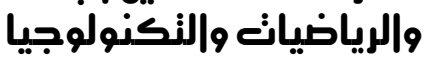

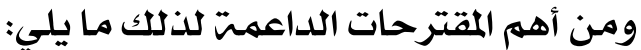

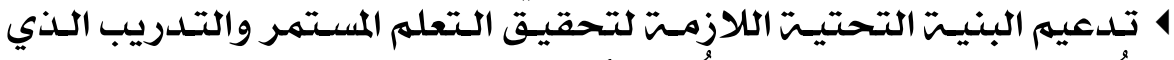

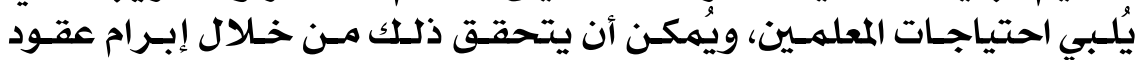

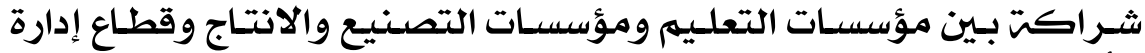

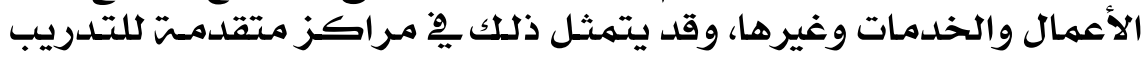

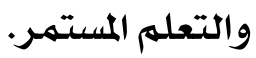

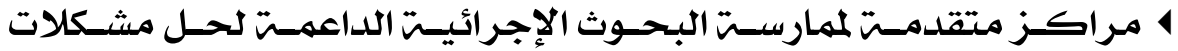

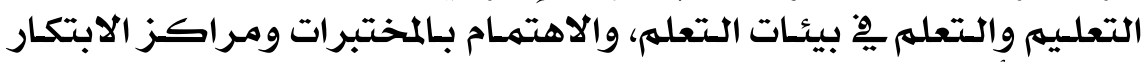

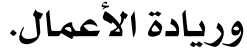

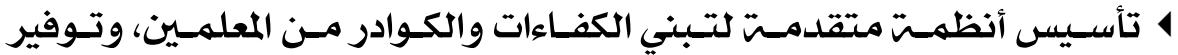

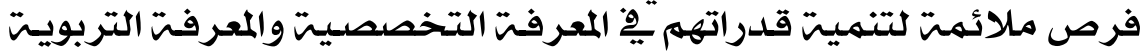

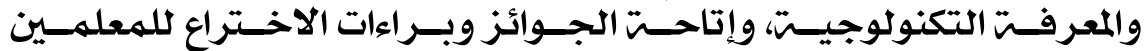

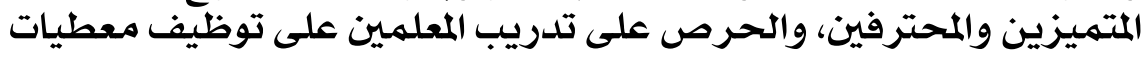

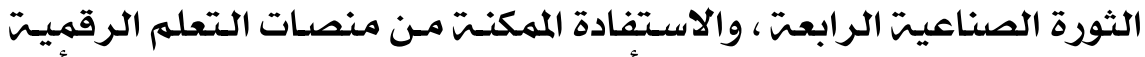

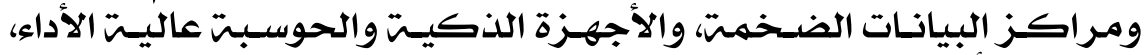
وانترنت الأشياء وغيرانير الفها.

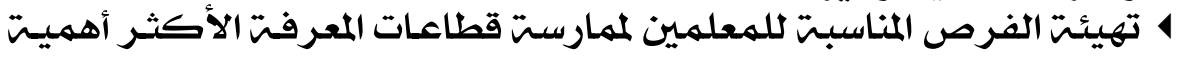

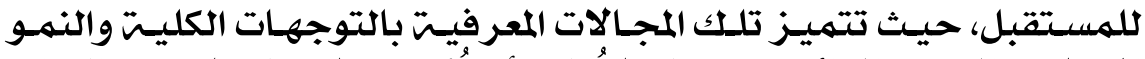

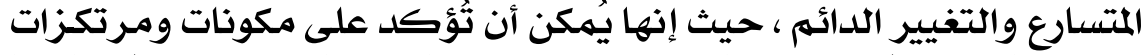

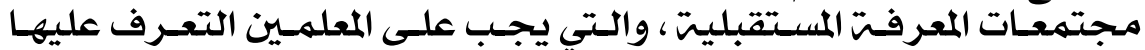

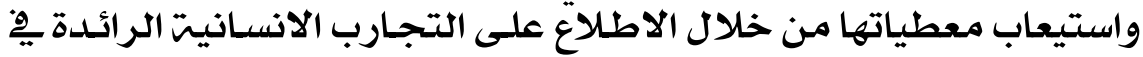

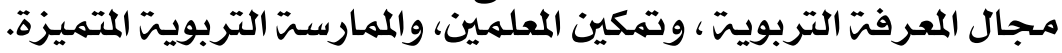

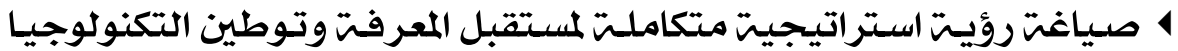

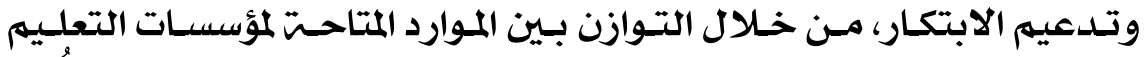

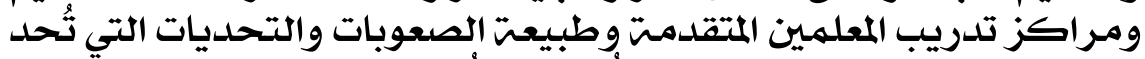

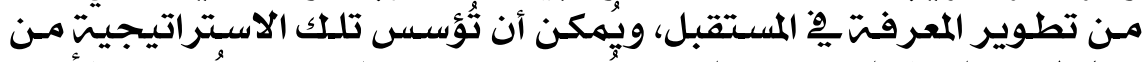

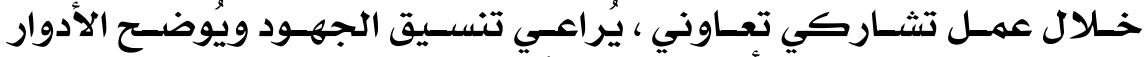

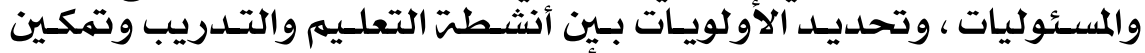

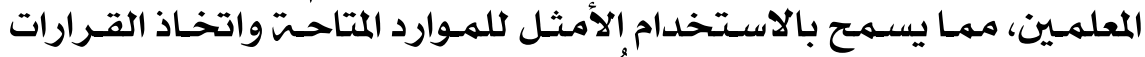

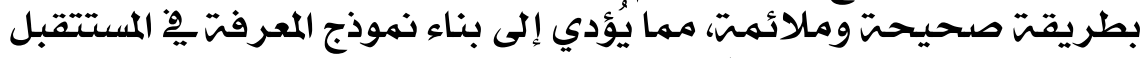

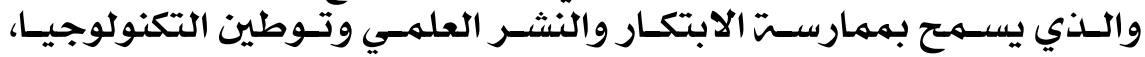

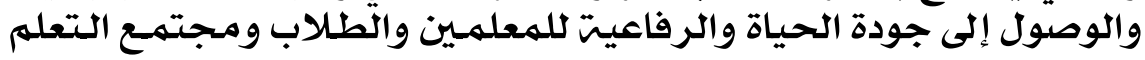




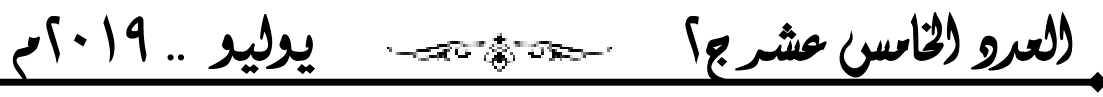

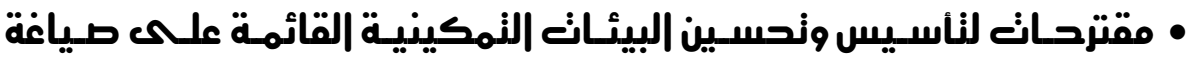

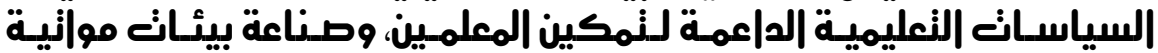

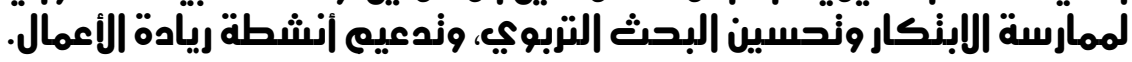

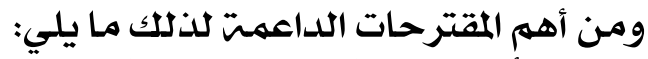

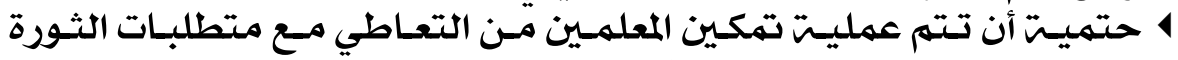

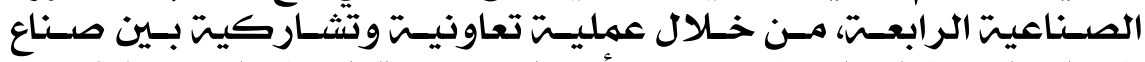

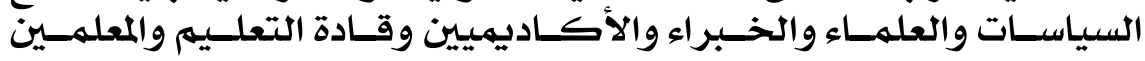

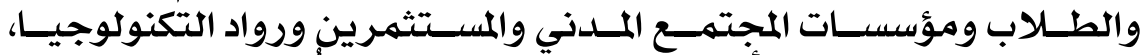

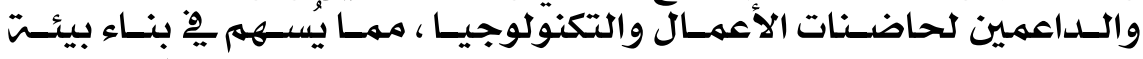

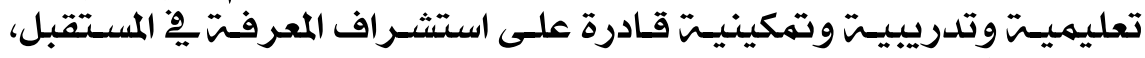

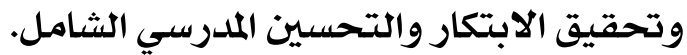

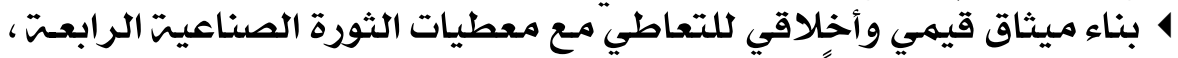

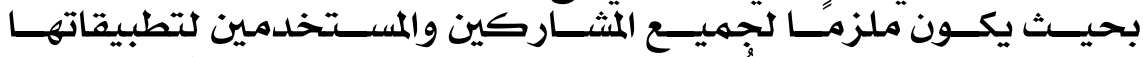

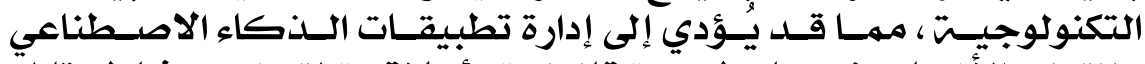

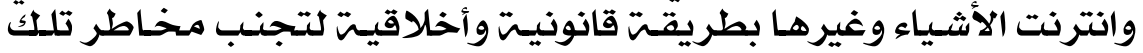

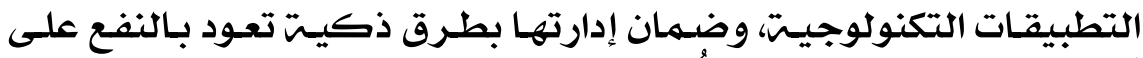

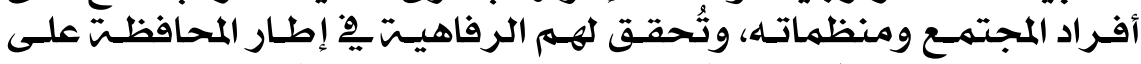

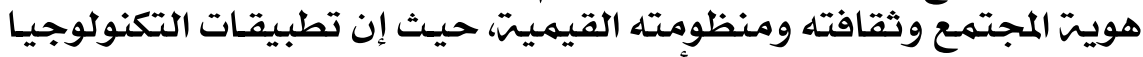

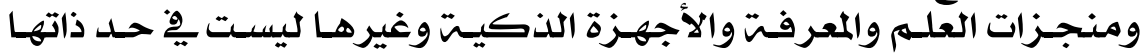

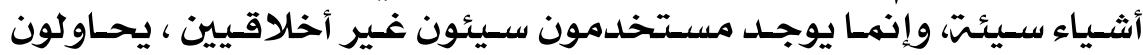

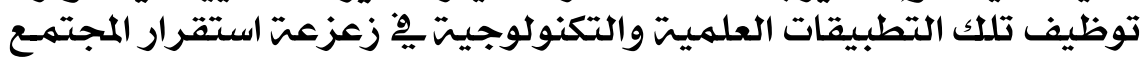

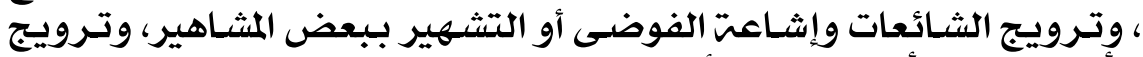

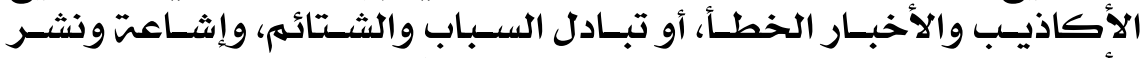

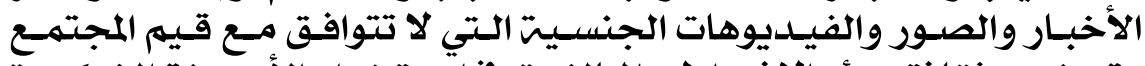

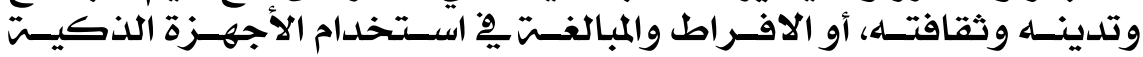

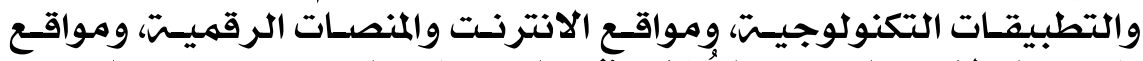

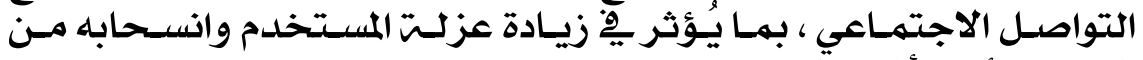

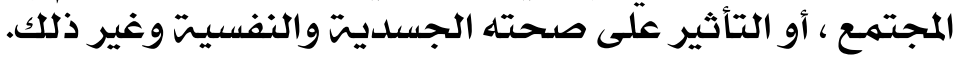

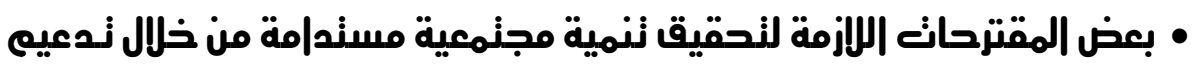

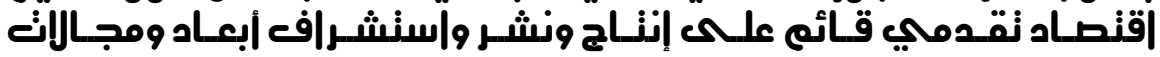

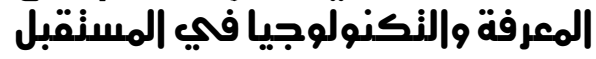

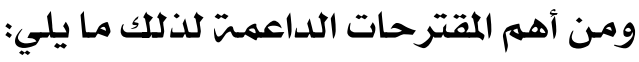

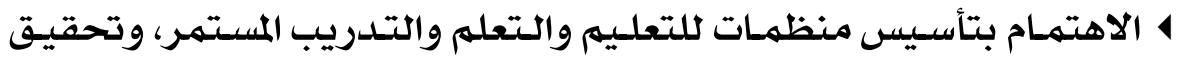

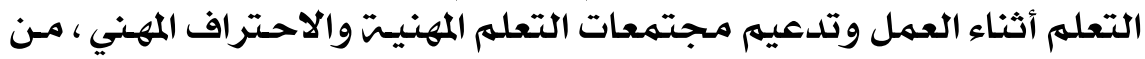

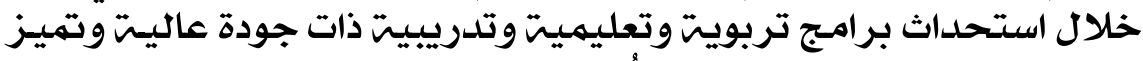

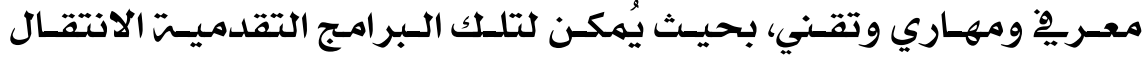

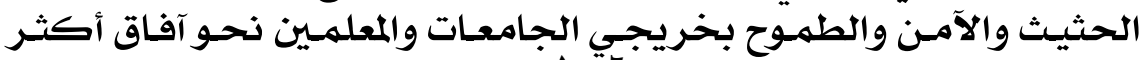
1.9 


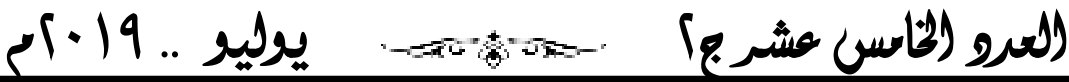

توافقا مـع مستقبل المعرفت وتطلعـات سـوق العهـل والانتـاج ، وإدارة الأعمـال

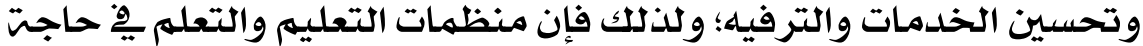

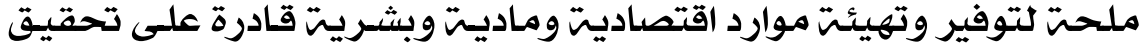

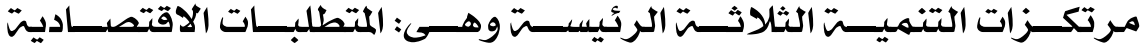

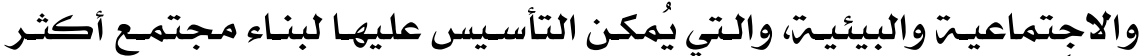
تقدمًا ورفاهيت.

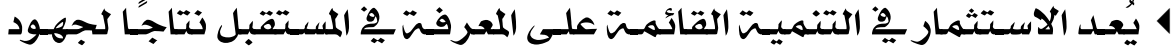

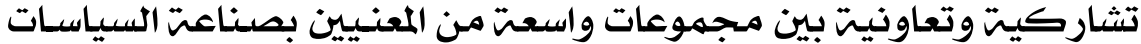

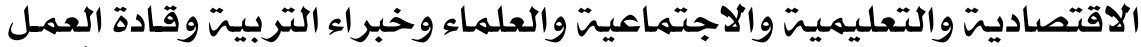

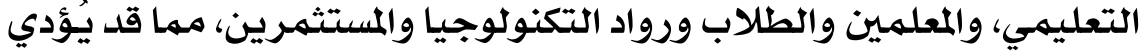

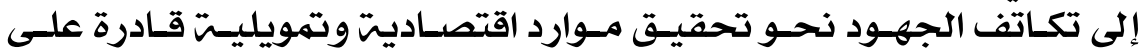

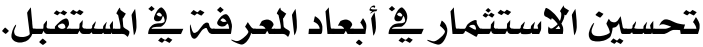

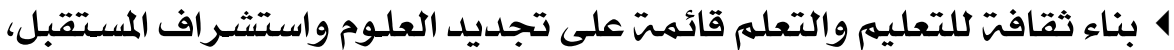

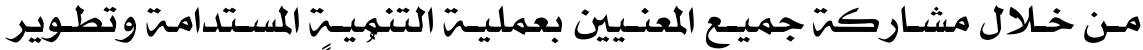

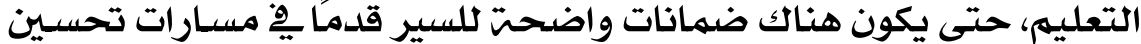

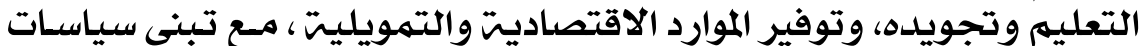

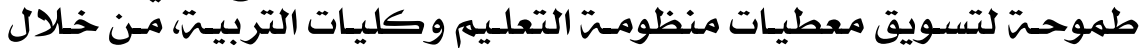

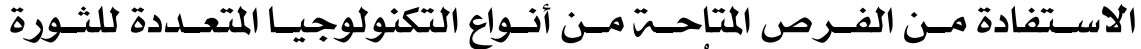

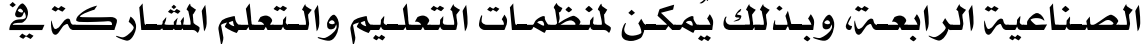

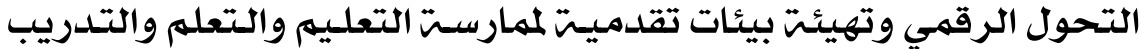

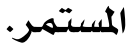

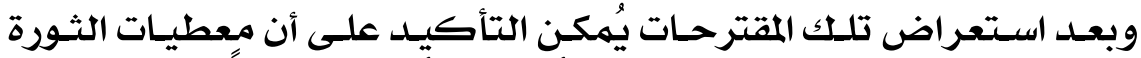

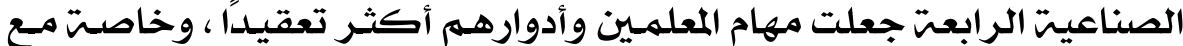

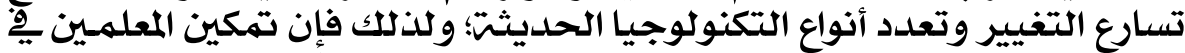

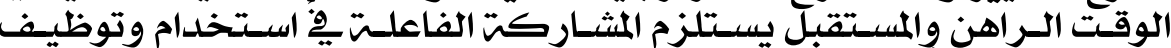

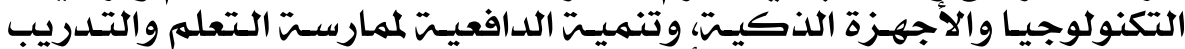

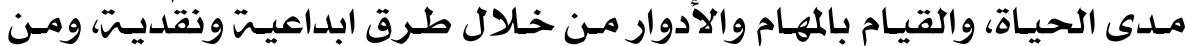

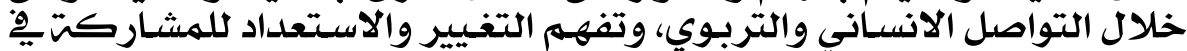

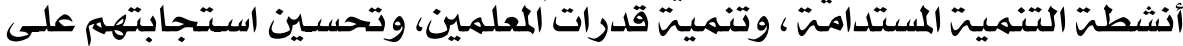

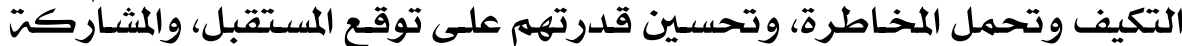

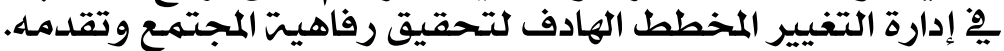

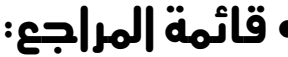

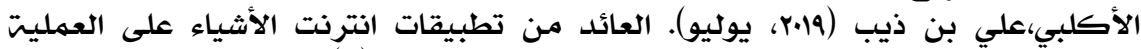

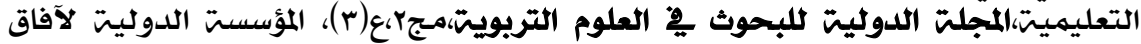

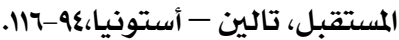

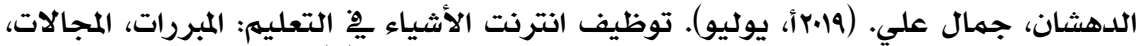

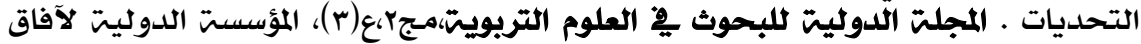




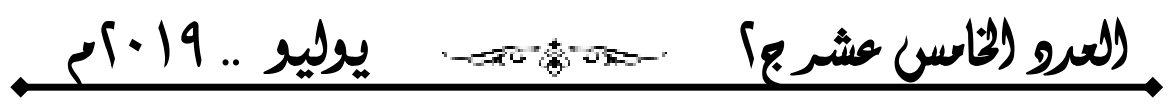

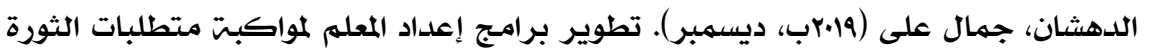

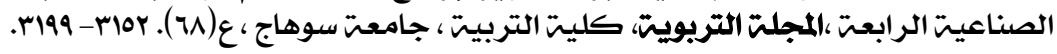

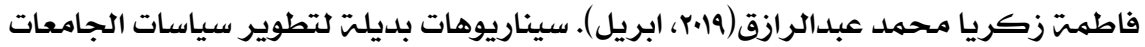

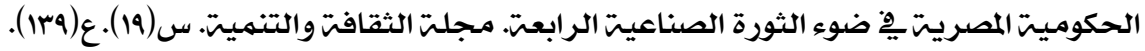

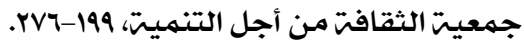

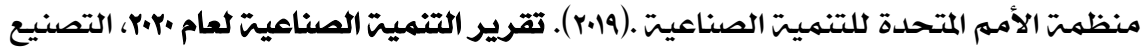

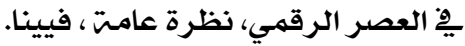

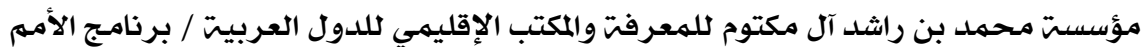

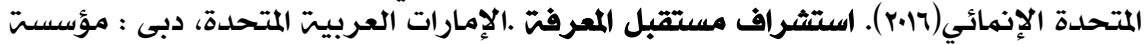
محمد بن راشد آل مكتوم للهمعرفتئ.

- Arvanitidi, E.; Drosos, C.; Theocharis, E.; Papoutsidakis, M.(2019, December). 3D Printing and Education . International Journal of Computer Applications(0975-8887), Vol.177, No.24,55-59.

- Brown-martin,g(2017). Education and the fourth industrial Revolution. UK; Groube Media tfo.

- Harrington, J. (2018). On the usefulness of "value" in the drfinition of creativity: A commentary. Creativity Research Journal، 30(1)، 118-121.

- Jagirdar, S.(2012, July). Cloud Computing Basics. International Journal of Advanced Research in Computer and Communication Engineering, Vol.1, Issue 5, 343-347.

- Machis, D. (2019). Mind the gaps in your professional development, Contract Management، 59، 87-88. Retrieved from https://search.proquest.com/docview/ 2173405575 ? accountid $=63189$

-Bernard Marr: 8 Things Every School Must Do To Prepare for the $4^{\text {th }}$ Industerial Revolution Retreaved From https://www.forbes. com/sites/bernardmarr/2019/05/22/8-things-every-school-must-do-to-preparefor-the-4th-industrial-revolution/\#1fccd8ab670c

- Pombo, C. ; Gupta, R. ; Stankovic, M.(2018). Social Services for digital Citizens Opportunities for Latin America\& the Caribbean. Inter- American Development Bank Felipe Herrera Liberary.

- Salazar, C.; Patel , K; Patel, S.(2016,May). Internet of Things IOT: Definition, Characteristics, Architecture, Enabling Technologies, Application \& Future Challenges. International Journal of Engineering Science and Computing. Vol.6, No.(5), 6122-6130.

- Schwab, K. (2016). The Fourth Industerial Revolution . Switzerland: World Economic Forum.

- Shahroom, A. A \& Hussin, N. (2018. September). Industerial Revolution 4.0 and Education. International Journal of Academic Research in Business and Social Sciences,8(9), 
(العرو (لإساس) عشرج) .

Human Resource Management Academic Research Society, 314319.

- Sike, J. P.‘\& Hess, A. N. (2014). Win-win professional development: Providing meaningful professional development while meeting the needs of all stakeholders.TechTrends, 58(6), 99108. doi:http://dx.doi.org/10.1007/s 11528-014-0809-7

- Verina, N.; Titko , J. (2019). Digital Transformation: Conceptual Framework. International Scientific Conference. Contemporary Issues in Business Management and Economics Engineering. 9-10 May, Vilnius, Lithuania. Vilnius Gediminas Technical University, 718-727.

- Venter, A. A. J., Herbst, T. H. H., \& Iwu, C. G. (2019). What will it take to make a successful administrative professional in the fourth industrial revolution? SA Journal of Human Resource Management · 17 doi:http://dx.doi.org/10.4102/sajhrm.v17i0.1224.

- Zouein, P (2019). Higher education on 4.0 Drivers and Framework, Expert Group Meeting on " Artificial Intelligence and Local Industrial Development, Lebanese American University, UN-House, Beirut.

at is

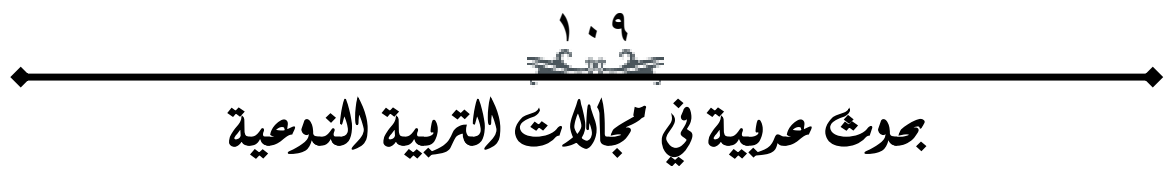


